\title{
ARL AnNual Salary Survey 2009-2010
}

Compiled and Edited by

Martha KyRILLIDOU

Les BlAND

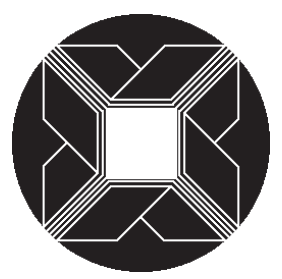

Association of ResEARCh LibraRIES

WASHINGTON, DC 
ARL Annual Salary Survey 2009-2010

The quantitative tables presented in this publication are not indicative of performance and should not be used as measures of library quality. In comparing any individual library to ARL medians or to other ARL members, one must be careful to make such comparisons within the context of differing institutional characteristics.

Custom reports based on the Salary Survey data are also available. Contact the ARL Statistics and Measurement Program Officer for further information.

Visit the ARL Statistics and Measurement Program online at http://www.arl.org/stats/.

Published by the

Association of Research Libraries

Washington, DC 20036

www.arl.org

ISSN 0361-5669

ISBN 1-59407-856-4

EAN 978-1-59407-856-9

(C) 2010

The compilation is copyrighted by the Association of Research Libraries. Blanket permission is granted to reproduce and distribute copies of this work for nonprofit, educational, or library purposes, provided that the author, source, and copyright notice are included on each copy. This permission is in addition to rights of reproduction granted under Sections 107, 108, and other provisions of the US Copyright Act.

The paper used in this publication meets the minimum requirements of the American National Standard for Information Science and National Information Standards Organization standard-Permanence of Paper for Publications and Documents in Libraries and Archives, ANSI/NISO Z39.48-1992(R1997). 


\section{CONTENTS}

Salary Survey Trends 2009-2010

Figure 1: $\quad$ Ethnicity/Race of Professional Staff in US ARL University Libraries, FY 2009-2010............ 8

Figure 2: $\quad$ Minority Professionals by Region in US ARL University Libraries, FY 2009-2010 …........... 9

Figure 3: Race/Ethnicity and Sex Distribution of Professional Staff in ARL University

Libraries, FY 2009-2010 10

Figure 4: Distribution of Functional Specialist Job Sub-Codes by Type of Library .12

Figure 5: Distribution of Functional Specialist Job Sub-Codes' Average Salary by Sex . .12

Figure 6: $\quad$ Average Salaries and Average Years of Experience of Library Professionals in Libraries with Three, Four, and Five Step Rank Structures, FY 2009-2010..... .14

\section{Salary LeVels for Staff in ARL Libraries}

Table 1: $\quad$ Distribution by Salary Level 19

Table 2: $\quad$ Salary Trends in ARL University Libraries 20

Table 3: $\quad$ Salary Trends in US ARL University Libraries. .21

Table 4: $\quad$ Salary Trends in Canadian ARL University Libraries 22

\section{ARL NONUNIVERSITY LibraRIES}

Table 5: Median and Beginning Professional Salaries in ARL Nonuniversity Libraries 25

Table 6: $\quad$ Salary Trends in ARL Nonuniversity Libraries .26

\section{ARL UniVersity LibraRIES}

Table 7: $\quad$ Filled Positions; Average, Median, Beginning Professional Salaries; and Average Years of Experience in ARL University Libraries, FY 2009-2010

Table 8: $\quad$ Beginning Professional Salaries in ARL University Libraries; Rank Order Table, FY 2008-2009 30

Table 9: $\quad$ Beginning Professional Salaries in ARL University Libraries; Rank Order Table, FY 2009-2010 
Table 10: $\quad$ Median Professional Salaries in ARL University Libraries;

Table 11: $\quad$ Median Professional Salaries in ARL University Libraries;

Rank Order Table, FY 2009-2010 33

Table 12: $\quad$ Average Professional Salaries in ARL University Libraries;

Rank Order Table, FY 2008-2009 34

Table 13: $\quad$ Average Professional Salaries in ARL University Libraries;

Rank Order Table, FY 2009-2010 35

Table 14: $\quad$ Average, Median, and Beginning Professional Salaries in ARL University

Libraries; Summary of Rankings, FY 2006-2007 to 2009-2010 36

Table 15: $\quad$ Distribution of Professional Staff in ARL University Libraries by Salary and Position, FY 2009-2010.

Table 16: Distribution of Professional Staff in ARL University Libraries by Salary, Sex, and Position, FY 2009-2010.

Table 17: $\quad$ Number and Average Salaries of ARL University Librarians by Position and Sex, FY 2009-2010 .

Table 18: $\quad$ Number and Average Years of Experience of ARL University Librarians by Position and Sex, FY 2009-2010.....

Table 19: $\quad$ Number and Average Salaries of ARL University Librarians by Years of Experience and Sex, FY 2009-2010

Table 20: $\quad$ Average Salaries of ARL University Librarians

by Position and Years of Experience, FY 2009-2010

Table 21: $\quad$ Number and Average Salaries of ARL University Librarians

by Position and Type of Institution, FY 2009-2010. 46

Table 22: $\quad$ Years of Experience of ARL University Librarians by Position and Type of Institution, FY 2009-2010.

Table 23: $\quad$ Number and Average Salaries of ARL University Librarians

by Position and Size of Professional Staff, FY 2009-2010. 48

Table 24: $\quad$ Years of Experience of ARL University Librarians by Position and Size of Professional Staff, FY 2009-2010. 49

Table 25: $\quad$ Average Salaries of ARL University Librarians by Position and Geographic Region, FY 2009-2010 50 


\section{US ARL UNIVERSITY LIBRARIES}

Table 26: $\quad$ Average Salaries of US ARL University Librarians by Position and Years of Experience, FY 2009-2010

Table 27: $\quad$ Number and Average Salaries of Minority US ARL University Librarians by Position and Sex, FY 2009-2010

Table 28: $\quad$ Number and Average Years of Experience of Minority US ARL University

Librarians by Position and Sex, FY 2009-2010

Table 29: $\quad$ Number and Average Salaries of US ARL University Librarians by Years of Experience and Sex, FY 2009-2010

Table 30: $\quad$ Number and Average Salaries of Minority US ARL University Librarians by Years of Experience and Sex, FY 2009-2010

\section{CANAdian ARL University LibRARIES}

Table 31: $\quad$ Filled Positions; Average, Median, and Beginning Professional Salaries; and Average Years of Professional Experience in Canadian ARL University Libraries, FY 2009-2010.. 60

Table 32: $\quad$ Number and Average Salaries of Canadian ARL University

Librarians by Position and Sex, FY 2009-2010 61

Table 33: $\quad$ Number and Average Years of Experience of Canadian ARL

University Librarians by Position and Sex, FY 2009-2010

Table 34: $\quad$ Number and Average Salaries of Canadian ARL University Librarians

by Years of Experience and Sex, FY 2009-2010 63

\section{ARL University Medical Libraries}

Table 35: $\quad$ Filled Positions; Average, Median, Beginning Professional Salaries; and Average Years of Professional Experience in ARL University Medical Libraries, FY 2009-2010...... 66

Table 36: Beginning Professional Salaries in ARL University Medical Libraries; Rank Order Table, FY 2009-2010

Table 37: $\quad$ Median Professional Salaries in ARL University Medical Libraries; Rank Order Table, FY 2009-2010 
Table 38: $\quad$ Average Professional Salaries in ARL University Medical Libraries;

Rank Order Table, FY 2009-2010 .70

Table 39: $\quad$ Number and Average Salaries of ARL University Medical Librarians

by Position and Sex, FY 2009-2010 71

Table 40: $\quad$ Number and Average Years of Experience of ARL University Medical Librarians by Position and Sex, FY 2009-2010 72

Table 41: $\quad$ Number and Average Salaries of ARL University Medical Librarians

by Years of Experience and Sex, FY 2009-2010 73

\section{ARL UNIVERSITY LAW LiBRARIES}

Table 42: $\quad$ Filled Positions; Average, Median, Beginning Professional Salaries; and Average Years of Experience in ARL University Law Libraries, FY 2009-2010. .76

Table 43: $\quad$ Beginning Professional Salaries in ARL University Law Libraries; Rank Order Table, FY 2009-2010 .78

Table 44: $\quad$ Median Professional Salaries in ARL University Law Libraries;

Rank Order Table, FY 2009-2010 79

Table 45: $\quad$ Average Professional Salaries in ARL University Law Libraries; Rank Order Table, FY 2009-2010 80

Table 46: $\quad$ Number and Average Salaries of ARL University Law Librarians by Position and Sex, FY 2009-2010. .81

Table 47: $\quad$ Number and Average Years of Experience of ARL University Law Librarians by Position and Sex, FY 2009-2010..... .82

Table 48: $\quad$ Number and Average Salaries of ARL University Law Librarians by Years of Experience and Sex, FY 2009-2010 83

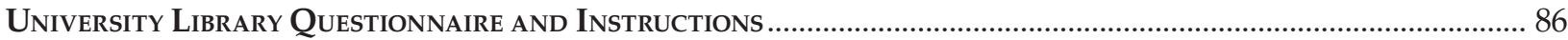

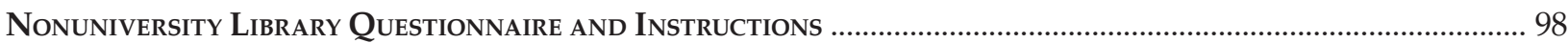

Footnotes to the ARL AnNual Salary SuRvey, 2009-2010 .............................................................................103

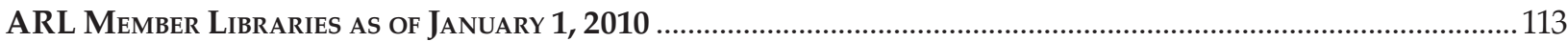

Table Numbering Changes for 1998-1999 to 1999-2000. 117 


\section{SALARY Survey Trends 2009-2010}

The ARL Annual Salary Survey 2009-2010 reports salary data for all professional staff working in ARL libraries. The Association of Research Libraries (ARL) represents the interests of libraries that serve major North American research institutions. The Association operates as a forum for the exchange of ideas and as an agent for collective action to influence forces affecting the ability of these libraries to meet the future needs of scholarship. The ARL Statistics and Measurement program, which produces the Salary Survey, is organized around collecting, analyzing, and distributing quantifiable information describing the characteristics of research libraries. The ARL Annual Salary Survey is the most comprehensive and thorough guide to current salaries in large US and Canadian academic and research libraries, and is a valuable management and research tool.

Data for 10,207 professional staff members were reported this year for the 114 ARL university libraries, including their law and medical libraries (948 staff members reported by 73 medical libraries and 747 staff members reported by 76 law libraries). For the 10 nonuniversity ARL members, data were reported for 3,811 professional staff members.

The tables are organized in seven major sections. The first section includes Tables 1 through 4 , which report salary figures for all professionals working in ARL member libraries, including law and medical library data. The second section includes salary information for the 10 nonuniversity research libraries of ARL. The third section, entitled "ARL University Libraries," reports data in Tables 7 through 25 for the "general" library system of the university ARL members, combining US and Canadian data but excluding law and medical data. The fourth section, composed of Tables 26 through 30, reports data on US ARL university library members excluding law and medical data; the fifth section, Tables 31-34, reports data on Canadian ARL university libraries excluding law and medical data. The sixth section (Tables 35-41) and the seventh section (Tables 42-48) report on medical and law libraries, respectively, combining US and Canadian data.

The university population is generally treated in three distinct groups: staff in the "general" library system, staff in the university medical libraries, and staff in the university law libraries. Any branch libraries for which data were received, other than law and medical, are included in the "general" category, whether or not those libraries are administratively independent. Footnotes for many institutions provide information on branch inclusion or exclusion.

In all tables where data from US and Canadian institutions are combined, Canadian salaries are converted into US dollar equivalents at the rate of 1.1667 Canadian dollars per US dollar. Tables 4 and 31 through 34, however, pertain exclusively to staff in Canadian university libraries, so salary data in those tables are expressed in Canadian dollars.

\footnotetext{
${ }^{1}$ This is the average monthly noon exchange rate published in the Bank of Canada Review for the period July 2008-June 2009 and is used in converting figures that are shown effective as of 1 July 2009. This information can be accessed at: http:// www.bankofcanada.ca/en/rates/exchange.html.
} 


\section{RACE AND ETHNICITY}

There were 1,296 minority professional staff reported in 99 US ARL university libraries, including law and medical. ${ }^{2}$ Note that the data for minority professionals comes only from the US ARL university libraries following the Equal Employment Opportunity Commission (EEOC) definitions; Canadian law prohibits the identification of Canadians by ethnic category.

Currently, $14.3 \%$ of the professional staff in US ARL university libraries (including law and medical) belongs to one of the four non-Caucasian categories for which ARL keeps records. The percentage of minorities in managerial or leadership positions in the largest US academic libraries is far lower: $6.1 \%$ are directors (7 out of 114), $6.3 \%$ are associate directors (20 out of 316), 5.3\% are assistant directors ( 9 out of 170 ) and $9.4 \%$ (46 out of 487) are branch librarians (see Table 27). Figure 1, below, depicts the overall racial/ethnic distribution of professional staff in US ARL university libraries: Caucasian/Other 85.7\%, Asian/Pacific Islander 6.4\%, Black 4.6\%, Hispanic $2.8 \%$, and American Indian/Alaskan Native $0.5 \%$.

\section{Figure 1: Ethnicity/Race of Professional Staff in US ARL University Libraries, FY 2009-2010}

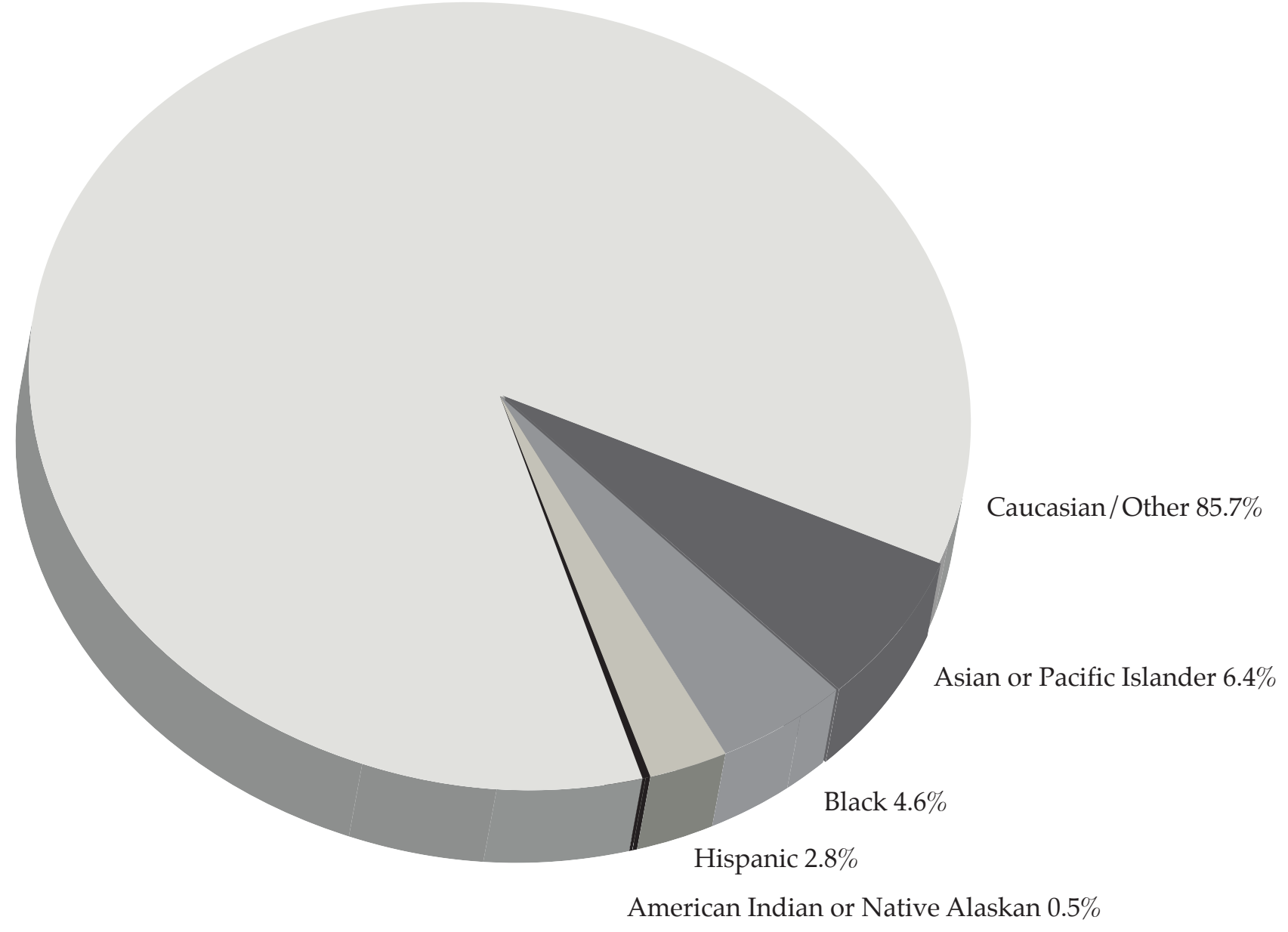

\footnotetext{
${ }^{2}$ Some US institutions offer their librarians the option of not reporting race and ethnicity; others forbid the tracking of racial and ethnic classification altogether. See Footnotes.
} 
Minority professional staff in US ARL university libraries continue to be disproportionately distributed across the country. Using Figure 1, we can compare the number of minority staff with other staff, region by region. These patterns of distribution have been relatively stable for the entire history of ARL's data-collection experience. Minorities are most underrepresented in the East South Central, New England, and Mountain regions (see page 51 for a definition of the regions). Proportionately to other regions, there are more minorities in the Pacific, South Atlantic, and West South Central.

Figure 2: Minority Professionals by Region in US ARL University Libraries, FY 2009-2010

\begin{tabular}{|c|c|c|c|c|c|c|c|c|c|c|c|}
\hline & $\begin{array}{l}\text { New } \\
\text { England }\end{array}$ & $\begin{array}{l}\text { Middle } \\
\text { Atlantic }\end{array}$ & $\begin{array}{c}\text { East } \\
\text { North } \\
\text { Central }\end{array}$ & $\begin{array}{l}\text { West } \\
\text { North } \\
\text { Central }\end{array}$ & $\begin{array}{l}\text { South } \\
\text { Atlantic }\end{array}$ & $\begin{array}{c}\text { East } \\
\text { South } \\
\text { Central }\end{array}$ & $\begin{array}{c}\text { West } \\
\text { South } \\
\text { Central }\end{array}$ & Mountain & Pacific & TOTAL & $\%$ \\
\hline \multirow{2}{*}{\multicolumn{12}{|c|}{$\begin{array}{l}\text { Race/Ethnicity } \\
\text { Category }\end{array}$}} \\
\hline & & & & & & & & & & & \\
\hline Black & 35 & 62 & 69 & 25 & 127 & 25 & 35 & 9 & 29 & 416 & $32 \%$ \\
\hline Hispanic & 23 & 41 & 31 & 11 & 38 & 5 & 41 & 20 & 43 & 253 & $20 \%$ \\
\hline Asian & 76 & 105 & 57 & 30 & 83 & 8 & 37 & 17 & 170 & 583 & $45 \%$ \\
\hline $\mathbf{A I} / \mathbf{A N}^{*}$ & 3 & 3 & 24 & 4 & 2 & & 2 & 6 & & 44 & $3 \%$ \\
\hline Minority Total & 137 & 211 & 181 & 70 & 250 & 38 & 115 & 52 & 242 & 1,296 & $100 \%$ \\
\hline \multicolumn{12}{|l|}{ Minority } \\
\hline Percent & $10.60 \%$ & $16.30 \%$ & $14.00 \%$ & $5.40 \%$ & $19.30 \%$ & $2.90 \%$ & $8.90 \%$ & $4.00 \%$ & $18.70 \%$ & & \\
\hline \multicolumn{12}{|l|}{ Nonminority } \\
\hline Total & 1,177 & 1,257 & 1,371 & 537 & 1,224 & 371 & 592 & 445 & 841 & 7,815 & $100 \%$ \\
\hline \multicolumn{12}{|l|}{ Nonminority } \\
\hline Percent & $15.10 \%$ & $16.10 \%$ & $17.50 \%$ & $6.90 \%$ & $15.70 \%$ & $4.70 \%$ & $7.60 \%$ & $5.70 \%$ & $10.80 \%$ & & \\
\hline \multicolumn{12}{|l|}{ Regional } \\
\hline \multicolumn{12}{|l|}{ Percent } \\
\hline Total staff & $14.40 \%$ & $16.10 \%$ & $17.00 \%$ & $6.70 \%$ & $16.20 \%$ & $4.50 \%$ & $7.80 \%$ & $5.50 \%$ & $11.90 \%$ & & \\
\hline \multicolumn{12}{|l|}{ Proportional } \\
\hline Minority & & & & & & & & & & & \\
\hline Representation & $-29.80 \%$ & $1.24 \%$ & $-20.00 \%$ & $-21.74 \%$ & $22.93 \%$ & $-38.30 \%$ & $17.11 \%$ & $-29.82 \%$ & $73.15 \%$ & & \\
\hline
\end{tabular}

* American Indian/Alaskan Native

ARL recognizes the difficulties that the profession has in attracting a diverse workforce and continues to work actively in the development of workplace climates that embrace diversity. The ARL Diversity Program, through its Leadership and Career Development Program and the Initiative to Recruit a Diverse Workforce, emphasizes ARL's and its members' commitment to creating a diverse academic and research library community to better meet the new challenges of global competition and changing demographics. Further, the Diversity 
Program focuses on issues surrounding work relationships in libraries while considering the impact of diversity on library services, interactions with library users, and the development of collections, at its homepage, http:// www.arl.org/diversity/.

Women comprise $68.2 \%$ of the four racial/ethnic groups that comprise minority staff, as compared to $62.7 \%$ of Caucasian/Other staff in all US ARL university libraries. The overall gender balance in the 114 Canadian and US university libraries (including law and medical) is 35.9\% male and 64.1\% female. See Figure 2, above, and Figure 3, below, for more detail on race/ethnic and gender distribution.

Figure 3: Race/Ethnicity and Sex Distribution of Professional Staff in ARL University Libraries, FY 2009-2010

\begin{tabular}{|c|c|c|c|c|c|}
\hline \multicolumn{6}{|c|}{ UNITED STATES } \\
\hline & \multicolumn{2}{|c|}{ Men } & \multicolumn{2}{|c|}{ Women } & \multirow[t]{2}{*}{ Total } \\
\hline & Number of Staff & Percent of Total & Number of Staff & Percent of Total & \\
\hline Main & 2,840 & $37.5 \%$ & 4,725 & $62.5 \%$ & 7,565 \\
\hline Medical & 251 & $29.7 \%$ & 595 & $70.3 \%$ & 846 \\
\hline Law & 239 & $33.9 \%$ & 466 & $66.1 \%$ & 705 \\
\hline Minority* & 412 & $31.8 \%$ & 884 & $68.2 \%$ & 1,296 \\
\hline Non-minority & 2,916 & $37.3 \%$ & 4,899 & $62.7 \%$ & 7,815 \\
\hline All & 3,328 & $36.5 \%$ & 5,783 & $63.5 \%$ & 9,111 \\
\hline \multicolumn{6}{|c|}{ CANADA } \\
\hline & \multicolumn{2}{|c|}{ Men } & \multicolumn{2}{|c|}{ Women } & Total \\
\hline & Number of Staff & Percent of Total & Number of Staff & Percent of Total & \\
\hline Main & 308 & $32.5 \%$ & 639 & $67.5 \%$ & 947 \\
\hline Medical & 11 & $10.8 \%$ & 91 & $89.2 \%$ & 102 \\
\hline Law & 14 & $33.3 \%$ & 28 & $66.7 \%$ & 42 \\
\hline All & 333 & $30.5 \%$ & 758 & $69.5 \%$ & 1,091 \\
\hline \multicolumn{6}{|c|}{ United STATES AND CANADA (Combined) } \\
\hline & \multicolumn{2}{|c|}{ Men } & \multicolumn{2}{|c|}{ Women } & Total \\
\hline & Number of Staff & Percent of Total & Number of Staff & Percent of Total & \\
\hline Main & 3,148 & $37.0 \%$ & 5,364 & $63.0 \%$ & 8,512 \\
\hline Medical & 262 & $27.6 \%$ & 686 & $72.4 \%$ & 948 \\
\hline Law & 253 & $33.9 \%$ & 494 & $66.1 \%$ & 747 \\
\hline All & 3,663 & $35.9 \%$ & 6,544 & $64.1 \%$ & 10,207 \\
\hline
\end{tabular}

* Includes staff in medical and law libraries.

Note: There are five entries from a US institution that did not report their race/ethnicity; therefore, the totals will not aggregate to the sum needed for the US and Canadian sub-totals to equal the figure displayed in the combined total. 


\section{GENDER DATA}

Many readers of previous surveys have inquired about evidence of gender-based salary differentials in ARL libraries. Additionally, data on salary comparisons for directors also are frequently requested. 2009-2010 repeated a trend observed in 2008-2009, the average salary for female directors was slightly higher than that of their male counterparts (see Table 17); furthermore, the number of women in the top administrative library position continued to increase (68 female directors out of 114 total director positions reported; compared to 63 out of 111 in 2008-2009).

However, salaries for women have not yet met parity with that of men (see Table 17): in 2009-2010 (just as in 2008-2009) the overall salary for women was only $96.2 \%$ that of men for the 114 ARL university libraries (compared to $95.39 \%$ in 2007-2008). This suggests a slow, long-term trend towards closure of the gender gap in ARL libraries — in 1980-1981 women in ARL libraries made roughly 87\% that of men.

Table 17 displays 27 job categories; females earn more than their male counterparts in just 10 of 27 categories listed. Table 18 provides average years of professional experience for many of the same staffing categories for which salary data are shown in Table 17, revealing that experience differentials may explain some differences within specific job categories. Women have more experience in all but three of the 10 job categories in which they average higher pay, but there are other categories in which women, on average, have more experience and less pay: Assistant Director, Functional Specialist, and Department Head - Other. Table 19 further reveals that the average salary for men is consistently higher than the average salary for women in nine of the ten experience cohorts, a pattern that is also repeated among minority librarians: the average salary for minority men is higher than that for minority women in eight of the ten experience cohorts (see Table 30).

There is a sense that the gender gap persists in academe in areas beyond the library and that a renewed commitment to resolve the problem is needed. ${ }^{3}$ A variety of reasons have been offered as to why these trends persist, most notably the perception that work is peripheral in a woman's life and, consequently, femaledominated professions are undervalued. Librarianship is predominantly and persistently a woman's profession. The scarcity of men in the profession has been well documented in many studies - the largest percentage of men employed in ARL libraries was 38.2\% in 1980-1981; since then men have consistently represented about 35\% of the professional staff in ARL libraries.

\section{The Functional Specialist Breakdown}

In 2004, the ARL Statistics and Measurement Committee accepted a proposal from the ACRL Personnel Administrators and Staff Development Officers Discussion Group to break down the Functional Specialist category (FSPEC). The group's major concern was that so many different types of positions, with their varying job descriptions and salaries, were being labeled with the code FSPEC that data reported for the category were beginning to lose meaning. For each position that would have been labeled FSPEC in past years, the proposal offered ARL institutions two options: either use one of eight new codes to describe that position; or, if none of the eight new codes could adequately describe that position, use FSPEC. As seen in Figure 4, only $15.6 \%$ of Functional Specialists in all libraries did not use an alternative code, a slight decrease from 17\% in 2008-2009. Of the positions that did use an alternate code, $63.2 \%$ were Archivists or Information Technology specialists.

\footnotetext{
${ }^{3}$ There are many instances citing the continuation of gender inequity in academia. See, for example: Denise K. Manger's articles in the Chronicle of Higher Education, "Faculty Salaries Increased 3.7\% in 1999-2000" (14 April 2000: A20) and "Faculty Salaries are Up 3.6\%, Double the Rate of Inflation" (23 April 1999: A16); D. W. Miller, "Salary Gap Between Male and Female Professors Grows Over the Years, Study Suggests," Chronicle of Higher Education, Today's News, 27 April 2000; and Yolanda Moses, "Salaries in Academe: The Gender Gap Persists," Chronicle of Higher Education 12 December 1997: A60.
} 
Figure 4: Distribution of Functional Specialist Job Sub-Codes by Type of Library

\begin{tabular}{|l|r|r|r|r|r|r|r|r|}
\hline \multirow{2}{*}{ Position } & \multicolumn{2}{|c|}{ Main } & \multicolumn{2}{c|}{ Medical } & \multicolumn{2}{|c|}{ Law } & \multicolumn{3}{|c|}{ All } \\
\cline { 2 - 9 } & \multirow{2}{*}{ No. } & Percent & No. & Percent & No. & Percent & No. & Percent \\
Archivists & & & & & & & & \\
Business Manager & 489 & $23.2 \%$ & 22 & $13.4 \%$ & 6 & $11.1 \%$ & 517 & $22.2 \%$ \\
Human Resources & 140 & $6.6 \%$ & 16 & $9.8 \%$ & 1 & $1.9 \%$ & 157 & $6.7 \%$ \\
IT, Systems & 90 & $4.3 \%$ & 1 & $0.6 \%$ & 0 & $0.0 \%$ & 91 & $3.9 \%$ \\
IT, Web Developer & 406 & $19.3 \%$ & 36 & $22.0 \%$ & 14 & $25.9 \%$ & 456 & $19.6 \%$ \\
IT, Programmer & 153 & $7.3 \%$ & 22 & $13.4 \%$ & 7 & $13.0 \%$ & 182 & $7.8 \%$ \\
Media Specialist & 289 & $13.7 \%$ & 28 & $17.1 \%$ & 0 & & 317 & $13.6 \%$ \\
Preservation & 102 & $4.8 \%$ & 6 & $3.7 \%$ & 4 & $7.4 \%$ & 112 & $4.8 \%$ \\
\hline Other Functional Specialists & 127 & $6.0 \%$ & 3 & $1.8 \%$ & 3 & $5.6 \%$ & 133 & $5.7 \%$ \\
\hline Total & 313 & $14.8 \%$ & 30 & $18.3 \%$ & 19 & $35.2 \%$ & 362 & $15.6 \%$ \\
\hline
\end{tabular}

Figure 5, below, displays the average salaries of the subcategories by position and sex (law and medical libraries not included) in the same fashion as Table 17. The salaries in each of the sub-categories deviate widely from the combined Functional Specialist average salary of $\$ 63,897$. Human resource specialists have the highest average of all subcategories, with an average salary of $\$ 71,840$; media/multimedia specialists have the lowest average salary of $\$ 54,794$.

Figure 5: Distribution of Functional Specialist Job Sub-Codes' Average Salaries by Sex

\begin{tabular}{|c|c|c|c|c|c|c|}
\hline \multirow{2}{*}{ Position } & \multicolumn{2}{|c|}{ Women } & \multicolumn{2}{|c|}{ Men } & \multicolumn{2}{|c|}{ Total } \\
\hline & Salary & No. & Salary & No. & Salary & No. \\
\hline Archivists & 57,541 & 306 & 60,534 & 183 & 58,661 & 489 \\
\hline Business Manager & 69,677 & 88 & 72,629 & 52 & 70,774 & 140 \\
\hline Human Resources & 70,826 & 75 & 76,907 & 15 & 71,840 & 90 \\
\hline IT, Systems & 65,346 & 142 & 64,944 & 264 & 65,085 & 406 \\
\hline IT, Web Developer & 60,787 & 67 & 63,836 & 86 & 62,500 & 153 \\
\hline IT, Programmer & 65,278 & 86 & 67,450 & 203 & 66,804 & 289 \\
\hline Media Specialist & 55,199 & 45 & 54,474 & 57 & 54,794 & 102 \\
\hline Preservation & 62,682 & 96 & 65,905 & 31 & 63,469 & 127 \\
\hline Other Functional Specialists & 60,351 & 201 & 62,577 & 112 & 61,148 & 313 \\
\hline All Functional Specialists & 63,076 & 1,106 & 65,473 & 1,003 & 63,897 & 2,109 \\
\hline
\end{tabular}

In regards to the gender gap in ARL libraries explained in the previous section, it is worth noting that the average salaries of men are higher than those of women in seven out of the nine categories in Figure 5. 


\section{Institutional Characteristics and Salaries}

\section{A. Public and Private Institutions}

The gap between salaries paid in private US ARL university libraries and those paid in publicly supported US university libraries decreased slightly; in 2008-2009 it was 7\%, in 2009-2010 the difference was 6\%, with an average of $\$ 4,120$ more paid for a position in a private institution. Out of 27 job categories, only in four (Heads of Documents/Maps, Heads of Rare Books/Manuscripts, Heads of Computer Systems and Other: Over 14 years experience) did librarians in public institutions earn more than their peers employed in private institutions (see Table 21).

\section{B. Library Size}

Library size, as measured by the number of professional staff, is another significant determinant of salary. As a rule, the largest libraries pay the highest average salaries, not only overall, but for specific positions as well. ${ }^{4}$ The largest libraries, those with more than 110 staff, reported the highest average salary, $\$ 72,765$, compared to $\$ 71,620$ for the libraries with between 75 and 110 staff (see Table 23). The smallest libraries (22 to 49 staff) had the third-highest average salary of the cohorts, followed by those libraries with 50 to 74 staff. The gap between the highest paying cohort and the lowest paying cohort remained unchanged, in 2009-2010 it was \$4,901, a difference of $6.8 \%$ percent (the difference in 2008-2009 was $\$ 4,994$, which was $7.2 \%$ ).

\section{Geographic Area}

In 2009-2010, the highest average salaries were found in New England $(\$ 76,371)$ followed by the Pacific $(\$ 75,283)$ region with Canadian salaries $(\$ 73,363)$ coming in third (see Table 25$)$. The reason for the drop in Canadian salaries relative to US institutions (highest in 2008-2009 at \$82,295) was the change in the currency exchange rate: in the 2008-2009 salary survey it was 1.0101; for the 2009-2010 survey period it is 1.1667. ${ }^{5}$ The East South Central region had the lowest average salary, with an average of $\$ 62,872$.

\section{Rank Structure}

Rank structure provides a useful framework for examining professional salaries in ARL university libraries. Figure 6, below, displays average salary and years of experience in the three most commonly used rank structures. Readers should be aware that not all individuals have a rank that fits into the rank structure the library utilizes. Most commonly, directors may have no rank (or a rank outside the structure) and it is common for non-librarians included in the survey (business officers, personnel staff, computer specialists, liaisons, etc.) to be unranked, as well.

The pattern of relationships between rank and salary seen in past years continues: with higher rank associated with higher average years of experience and a correspondingly higher salary. Of the 8,512 librarians in ARL university member libraries, 6,082 occupy a rank within these three most commonly found ranking systems, and the largest number of professionals $(3,223)$ occupy a position in a four-step rank structure.

\footnotetext{
${ }^{4}$ In 1995-1996, the largest cohort of libraries was determined based on staff over 124; in 1996-1998, over 120; in 1998-1999, over 115; and since 1999-2000, over 110. See Table 23.

${ }^{5}$ This is the average monthly noon exchange rate published in the Bank of Canada Review for the period July 2008-June 2009 and is used in converting figures that are shown effective as of 1 July 2009. This information can be accessed at: http:// www.bankofcanada.ca/en/rates/exchange.html.
} 
Figure 6: Average Salaries and Average Years of Experience of Library Professionals in Libraries with Three, Four, and Five Step Rank Structures, FY 2009-2010

\begin{tabular}{|c|c|c|c|c|c|c|}
\hline & \multicolumn{2}{|c|}{ Three-Step } & \multicolumn{2}{|c|}{ Four-Step } & \multicolumn{2}{|c|}{ Five-Step } \\
\hline & Salary & Experience & Salary & Experience & Salary & Experience \\
\hline Librarian 1 & 54,708 & 8.7 & 51,078 & 7.2 & 53,339 & 8.7 \\
\hline Librarian 2 & 66,402 & 17.4 & 57,281 & 11.2 & 60,497 & 12.1 \\
\hline Librarian 3 & 84,896 & 25.4 & 70,677 & 20.5 & 70,433 & 17.8 \\
\hline Librarian 4 & & & 86,032 & 26.4 & 86,431 & 23.7 \\
\hline Librarian 5 & & & & & 102,257 & 30.0 \\
\hline No. of Staff & \multicolumn{2}{|c|}{1,883} & \multicolumn{2}{|c|}{3,223} & \multicolumn{2}{|c|}{976} \\
\hline
\end{tabular}

\section{INFLATION EFFECT}

Tables 2 and 6 reveal changes in beginning professional and median salaries as reported by both university and nonuniversity research libraries as well as the US Bureau of Labor's Cost of Living Index (CPI-All Urban Consumers). Table 3 is similar to Table 2, but reports data only on US libraries. Table 4 shows trend data for Canadian libraries and compares them to the changes in the Canadian Consumer Price Index (Consumer Price Index for Canada, all-items, not seasonally adjusted). Tables 2, 3, and 4 include law and medical library staff in ARL university libraries. In contrast to 2007-2008, these tables indicate that the purchasing power of professionals (in both the United States and Canada) employed in ARL libraries outpaced inflation.

The median salary for US ARL university libraries in 2009 was $\$ 64,069$, an increase of $0.6 \%$ over the 2008-2009 median salary of $\$ 63,673$ (see Table 3). This modest salary increase compared favorably to the severe economic contraction of the same period, which saw the US CPI drop 2\% (see Table 3). ${ }^{6}$ Canadian salaries (when judged in Canadian dollars) were even more successful in surpassing inflation: while the Canadian CPI dropped $-0.9 \%$, median salaries in Canadian university libraries increased from $\$ 78,742$ (Canadian) to $\$ 80,654$ (Canadian) a rise of 2.4\% (see Table 4). ${ }^{7}$ However, the sharp difference in the exchange rates between 2008-2009 (1.0101 Canadian per US dollar) and 2009-2010 (1.1667 Canadian per US dollar), when judged in US currency, caused the Canadian median salary to drop from $\$ 77,954$ in 2008-2009 to $\$ 69,130$ - a decrease of $11.4 \%$ (see Table 4). This change in currency exchange rate is also responsible for the drop in overall (US and Canadian) median salary of ARL university librarians (see Table 2) from $\$ 64,823$ (US dollars) in 2008-2009 to $\$ 64,560$ (US dollars), which was a decrease of $0.5 \%$ despite the fact that both US and Canadian salaries increased modestly in 2009-2010 as stated above.

The median beginning salary (BPS) for university ARL librarians decreased during this survey's period: it was $\$ 44,000$ (US) in 2008-2009 and dropped to $\$ 43,700$ in 2009-2010 (a decrease of 0.7\%) (see Table 2). Table 6 shows that nonuniversity librarians also experienced a decrease in their beginning salary, which was $\$ 48,108$ in 2008-2009 and dropped to \$47,554 in 2009-2010 (a difference of 1.2\%). Table 6 also demonstrates that the median

\footnotetext{
${ }^{6}$ CPI data retrieved from the US Department of Labor, Bureau of Labor Statistics' Consumer Price Index-All Urban Consumers (US All items, 1982-84=100 - CUUR0000SA0) available online at http://www.bls.gov/data/.

7 The source for Canadian CPI data is "Table 5: The Consumer Price Index for Canada (All-Items, Not Seasonally Adjusted, Historical Data)" published in The Daily, a Statistics Canada publication, available online at http://www.statcan.gc.ca/ pub/62-001-x/2009010/t040-eng.htm.
} 
salaries of ARL nonuniversity librarians remained virtually the same: $\$ 85,320$ in $2008-2009$, \$85,229 in 2009-2010 — a decrease of $0.2 \%$.

Readers are reminded that these data reflect only salaries and that there are other compensation issues which may have influenced the pattern of salaries in various institutions. In addition, a highly standardized structure for capturing data has been used, which may portray results in a way that cannot be fully representative of a local situation.

Martha Kyrillidou

Les Bland

Association of Research Libraries

April 1, 2010 

Salary LeVels for Staff in ARL Libraries

TABles 1-4 



\section{Table 1: Distribution by Salary Level}

Figures in columns headed by fiscal year show the number of filled professional positions. Columns headed by Cum. \% show the percentage of all filled positions with salaries equal to or more than the beginning of each salary range. For example: in FY 2008-2009, 82.8\% of all ARL university librarians earned more than $\$ 50,000$, as did $96 \%$ of all ARL nonuniversity librarians.

Note: Canadian salaries are expressed in US dollars. Data includes medical and law libraries.

\begin{tabular}{|c|c|c|c|c|c|c|c|c|}
\hline \multirow[b]{3}{*}{ Salary Range } & \multicolumn{4}{|c|}{ University Librarians } & \multicolumn{4}{|c|}{ Nonuniversity Librarians } \\
\hline & FY & Cum. & FY & Cum. & FY & Cum. & FY & Cum. \\
\hline & 2008-2009 & $\%$ & 2009-2010 & $\%$ & 2008-2009 & $\%$ & 2009-2010 & $\%$ \\
\hline 300,000 and above & 7 & $0.1 \%$ & 6 & $0.1 \%$ & & $0.0 \%$ & & $0.0 \%$ \\
\hline $250,000-299,999$ & 10 & $0.2 \%$ & 12 & $0.2 \%$ & 1 & $0.0 \%$ & 1 & $0.0 \%$ \\
\hline $200,000-249,999$ & 36 & $0.5 \%$ & 37 & $0.5 \%$ & 2 & $0.1 \%$ & 3 & $0.1 \%$ \\
\hline 175,000 - 199,999 & 55 & $1.1 \%$ & 58 & $1.1 \%$ & 3 & $0.2 \%$ & 6 & $0.3 \%$ \\
\hline 150,000 - 174,999 & 91 & $2.0 \%$ & 80 & $1.9 \%$ & 112 & $3.1 \%$ & 284 & $7.7 \%$ \\
\hline 140,000 - 149,999 & 55 & $2.5 \%$ & 52 & $2.4 \%$ & 197 & $8.4 \%$ & 105 & $10.5 \%$ \\
\hline 130,000 - 139,999 & 87 & $3.4 \%$ & 80 & $3.2 \%$ & 115 & $11.5 \%$ & 237 & $16.7 \%$ \\
\hline 120,000 - 129,999 & 124 & $4.6 \%$ & 127 & $4.4 \%$ & 251 & $18.2 \%$ & 170 & $21.1 \%$ \\
\hline 110,000 - 119,999 & 229 & $6.8 \%$ & 193 & $6.3 \%$ & 237 & $24.5 \%$ & 370 & $30.9 \%$ \\
\hline 100,000 - 109,999 & 369 & $10.5 \%$ & 328 & $9.5 \%$ & 373 & $34.4 \%$ & 383 & $40.9 \%$ \\
\hline 95,000 - 99,999 & 296 & $13.4 \%$ & 287 & $12.3 \%$ & 241 & $40.9 \%$ & 301 & $48.8 \%$ \\
\hline $90,000-94,999$ & 296 & $16.3 \%$ & 337 & $15.6 \%$ & 312 & $49.2 \%$ & 144 & $52.6 \%$ \\
\hline $85,000-89,999$ & 413 & $20.4 \%$ & 418 & $19.7 \%$ & 195 & $54.4 \%$ & 276 & $59.8 \%$ \\
\hline $80,000-84,999$ & 568 & $26.0 \%$ & 546 & $25.1 \%$ & 244 & $60.9 \%$ & 201 & $65.1 \%$ \\
\hline $79,000-79,999$ & 110 & $27.1 \%$ & 121 & $26.3 \%$ & 60 & $62.5 \%$ & 48 & $66.4 \%$ \\
\hline $78,000-78,999$ & 119 & $28.2 \%$ & 143 & $27.7 \%$ & 59 & $64.1 \%$ & 48 & $67.6 \%$ \\
\hline $76,000-77,999$ & 250 & $30.7 \%$ & 261 & $30.2 \%$ & 60 & $65.7 \%$ & 49 & $68.9 \%$ \\
\hline $74,000-75,999$ & 322 & $33.9 \%$ & 321 & $33.4 \%$ & 130 & $69.2 \%$ & 89 & $71.2 \%$ \\
\hline $72,000-73,999$ & 299 & $36.8 \%$ & 285 & $36.2 \%$ & 88 & $71.5 \%$ & 123 & $74.5 \%$ \\
\hline $70,000-71,999$ & 301 & $39.8 \%$ & 323 & $39.3 \%$ & 92 & $74.0 \%$ & 29 & $75.2 \%$ \\
\hline $68,000-69,999$ & 410 & $43.8 \%$ & 400 & $43.3 \%$ & 114 & $77.0 \%$ & 97 & $77.8 \%$ \\
\hline $66,000-67,999$ & 351 & $47.3 \%$ & 370 & $46.9 \%$ & 40 & $78.1 \%$ & 89 & $80.1 \%$ \\
\hline $64,000-65,999$ & 473 & $51.9 \%$ & 451 & $51.3 \%$ & 123 & $81.4 \%$ & 89 & $82.4 \%$ \\
\hline $62,000-63,999$ & 365 & $55.5 \%$ & 400 & $55.2 \%$ & 83 & $83.6 \%$ & 114 & $85.4 \%$ \\
\hline $60,000-61,999$ & 523 & $60.7 \%$ & 530 & $60.4 \%$ & 114 & $86.6 \%$ & 93 & $87.9 \%$ \\
\hline $58,000-59,999$ & 420 & $64.8 \%$ & 430 & $64.6 \%$ & 159 & $90.8 \%$ & 46 & $89.1 \%$ \\
\hline $56,000-57,999$ & 466 & $69.4 \%$ & 487 & $69.4 \%$ & 46 & $92.1 \%$ & 120 & $92.2 \%$ \\
\hline $54,000-55,999$ & 441 & $73.8 \%$ & 459 & $73.9 \%$ & 34 & $93.0 \%$ & 44 & $93.4 \%$ \\
\hline $52,000-53,999$ & 475 & $78.4 \%$ & 496 & $78.7 \%$ & 65 & $94.7 \%$ & 84 & $95.6 \%$ \\
\hline $50,000-51,999$ & 441 & $82.8 \%$ & 439 & $83.1 \%$ & 47 & $96.0 \%$ & 87 & $97.9 \%$ \\
\hline $48,000-49,999$ & 417 & $86.9 \%$ & 446 & $87.4 \%$ & 66 & $97.7 \%$ & 13 & $98.2 \%$ \\
\hline $46,000-47,999$ & 361 & $90.5 \%$ & 368 & $91.0 \%$ & 30 & $98.5 \%$ & 16 & $98.6 \%$ \\
\hline $44,000-45,999$ & 321 & $93.6 \%$ & 326 & $94.2 \%$ & 7 & $98.7 \%$ & 11 & $98.9 \%$ \\
\hline $42,000-43,999$ & 217 & $95.8 \%$ & 217 & $96.3 \%$ & 24 & $99.4 \%$ & 14 & $99.3 \%$ \\
\hline $40,000-41,999$ & 182 & $97.6 \%$ & 175 & $98.1 \%$ & 3 & $99.4 \%$ & 17 & $99.7 \%$ \\
\hline $38,000-39,999$ & 109 & $98.6 \%$ & 85 & $98.9 \%$ & 9 & $99.7 \%$ & 0 & $99.7 \%$ \\
\hline $36,000-37,999$ & 60 & $99.2 \%$ & 52 & $99.4 \%$ & 0 & $99.7 \%$ & 2 & $99.8 \%$ \\
\hline $34,000-35,999$ & 43 & $99.6 \%$ & 38 & $99.8 \%$ & 0 & $\begin{array}{r}99.7 \% \\
\end{array}$ & 3 & $\begin{array}{r}99.9 \% \\
900 \%\end{array}$ \\
\hline less than 34,000 & 36 & $100.0 \%$ & 23 & $100.0 \%$ & 12 & $100.0 \%$ & 5 & $100.0 \%$ \\
\hline Total Positions & & 10,148 & & 10,207 & & 3,748 & & 3,811 \\
\hline Median Salary & & $\$ 64,828$ & & $\$ 64,560$ & & $\$ 80,320$ & & $\$ 85,229$ \\
\hline
\end{tabular}




\section{Table 2: Salary Trends in ARL University Libraries}

Salary figures for the current year are displayed in the context of previous years and compared to the changes in the US Consumer Price Index (CPI) to show trends in the purchasing power of median and beginning professional salaries. Salary figures and CPI numbers have been converted to adjusted indexes, using July 1984 as the base. Actual CPI data retrieved from the US Department of Labor, Bureau of Labor Statistics' Consumer Price Index-All Urban Consumers (US All items, 1982-1984=100 - CUUR0000SA0) available online at http://www. bls.gov/data/.

Note: Canadian salaries are expressed in US dollars.

\begin{tabular}{|c|c|c|c|c|c|c|c|c|}
\hline $\begin{array}{l}\text { Fiscal } \\
\text { Year }\end{array}$ & $\begin{array}{l}\text { Number of } \\
\text { Libraries }\end{array}$ & $\begin{array}{l}\text { Total } \\
\text { Staff }\end{array}$ & $\begin{array}{l}\text { Median } \\
\text { Salary }^{\dagger}\end{array}$ & $\begin{array}{c}\text { BPS }^{\ddagger} \\
\text { Median }\end{array}$ & $\begin{array}{c}\text { Median } \\
\text { Salary Index }\end{array}$ & $\begin{array}{c}\text { BPS }^{\ddagger} \\
\text { Index }\end{array}$ & $\begin{array}{l}\text { Actual } \\
\text { CPI* }^{*}\end{array}$ & $\begin{array}{l}\text { Adjusted } \\
\text { CPI }\end{array}$ \\
\hline 2009-2010 & 114 & 10,207 & $\$ 64,560$ & $\$ 43,700$ & 247.3 & 264.8 & 215.4 & 207.3 \\
\hline 2008-2009 & 113 & 10,148 & 64,823 & 44,000 & 248.4 & 266.7 & 219.9 & 211.6 \\
\hline 2007-2008 & 113 & 9,983 & 61,833 & 41,125 & 236.9 & 249.7 & 208.3 & 200.5 \\
\hline 2006-2007 & 113 & 9,824 & 59,648 & 40,000 & 228.5 & 242.4 & 203.5 & 195.9 \\
\hline 2005-2006 & 113 & 9,655 & 57,074 & 37,920 & 218.7 & 229.8 & 195.4 & 188.1 \\
\hline 2004-2005 & 113 & 9,487 & 55,250 & 36,984 & 211.7 & 224.1 & 189.4 & 182.3 \\
\hline 2003-2004 & 114 & 9,492 & 53,000 & 36,000 & 203.1 & 218.2 & 183.9 & 177.0 \\
\hline 2002-2003 & 114 & 9,469 & 51,636 & 35,000 & 197.8 & 212.1 & 180.1 & 173.3 \\
\hline 2001-2002 & 113 & 9,198 & 50,724 & 34,000 & 194.3 & 206.1 & 177.5 & 170.8 \\
\hline 2000-2001 & 112 & 8,882 & 49,068 & 32,879 & 188.0 & 199.3 & 172.8 & 166.3 \\
\hline 1999-2000 & 111 & 8,595 & 47,377 & 31,100 & 181.5 & 188.5 & 166.7 & 160.4 \\
\hline 1998-1999 & 110 & 8,400 & 45,775 & 30,000 & 175.2 & 181.7 & 163.2 & 157.1 \\
\hline 1997-1998 & 110 & 8,414 & 44,534 & 28,500 & 170.5 & 172.6 & 160.5 & 154.5 \\
\hline 1996-1997 & 109 & 8,325 & 43,170 & 27,687 & 165.3 & 167.7 & 157.0 & 151.1 \\
\hline 1995-1996 & 108 & 8,231 & 41,901 & 27,000 & 160.5 & 163.6 & 152.5 & 146.8 \\
\hline 1994-1995 & 108 & 8,216 & 41,088 & 26,000 & 157.4 & 157.6 & 148.4 & 142.8 \\
\hline 1993-1994 & 108 & 8,132 & 40,225 & 25,834 & 154.1 & 156.6 & 144.4 & 139.0 \\
\hline 1992-1993 & 108 & 8,212 & 39,265 & 25,000 & 150.4 & 151.5 & 140.5 & 134.9 \\
\hline 1991-1992 & 107 & 8,256 & 38,537 & 24,000 & 147.7 & 145.5 & 136.2 & 131.1 \\
\hline 1990-1991 & 107 & 8,382 & 36,701 & 23,800 & 140.6 & 144.2 & 130.4 & 125.8 \\
\hline 1989-1990 & 107 & 8,253 & 34,629 & 22,000 & 132.7 & 133.3 & 124.4 & 119.3 \\
\hline 1988-1989 & 107 & 8,087 & 32,461 & 20,400 & 124.4 & 123.6 & 118.5 & 113.9 \\
\hline 1987-1988 & 106 & 7,962 & 30,534 & 19,460 & 117.0 & 117.9 & 113.8 & 109.3 \\
\hline 1986-1987 & 105 & 7,718 & 28,941 & 18,250 & 110.9 & 110.6 & 109.5 & 105.5 \\
\hline 1985-1986 & 105 & 7,543 & 27,485 & 17,500 & 105.3 & 106.1 & 107.8 & 103.6 \\
\hline 1984-1985 & 104 & 7,161 & 26,100 & 16,500 & 100.0 & 100.0 & 104.1 & 100.0 \\
\hline
\end{tabular}

*Actual CPI figures have been revised from previous editions based upon changes published by the Bureau of Labor Statistics. These changes are minute, less than 0.3 in all cases.

† Includes medical and law libraries.

‡ Beginning professional salary. 


\section{Table 3: SAlary Trends in US ARL University Libraries}

Salary figures for the current year are displayed in the context of previous years and compared to the changes in the US Consumer Price Index (CPI) to show trends in the purchasing power of median and beginning professional salaries. Salary figures and CPI numbers have been converted to adjusted indexes, using July 1984 as the base. Actual CPI data retrieved from the US Department of Labor, Bureau of Labor Statistics' Consumer Price Index-All Urban Consumers (US All items, 1982-1984=100 - CUUR0000SA0) available online at http://www. bls.gov/data/.

\begin{tabular}{|c|c|c|c|c|c|c|c|c|}
\hline $\begin{array}{c}\text { Fiscal } \\
\text { Year }\end{array}$ & $\begin{array}{c}\text { Number of } \\
\text { Libraries }\end{array}$ & $\begin{array}{l}\text { Total } \\
\text { Staff }\end{array}$ & $\begin{array}{c}\text { Median } \\
\text { Salary }^{\dagger}\end{array}$ & $\begin{array}{c}\text { Median } \\
\text { Salary Change }\end{array}$ & $\begin{array}{c}\text { Median } \\
\text { Salary Index }\end{array}$ & $\begin{array}{c}\text { Actual } \\
\text { CPI* }^{*}\end{array}$ & $\begin{array}{c}\text { Adjusted } \\
\text { CPI }\end{array}$ & $\begin{array}{c}\text { CPI } \\
\text { Change }\end{array}$ \\
\hline 2009-2010 & 99 & 9,116 & $\$ 64,069$ & $0.6 \%$ & 246.9 & 215.4 & 207.3 & $-2.0 \%$ \\
\hline 2008-2009 & 99 & 9,158 & 63,673 & 3.8 & 245.4 & 219.9 & 211.6 & 5.6 \\
\hline $2007-2008$ & 99 & 9,026 & 61,329 & 3.5 & 236.4 & 208.3 & 200.5 & 2.4 \\
\hline $2006-2007$ & 99 & 8,866 & 59,280 & 3.7 & 228.5 & 203.5 & 195.9 & 4.1 \\
\hline $2005-2006$ & 99 & 8,700 & 57,173 & 2.8 & 220.4 & 195.4 & 188.1 & 3.2 \\
\hline 2004-2005 & 99 & 8,581 & 55,600 & 3.2 & 214.3 & 189.4 & 182.3 & 3.0 \\
\hline 2003-2004 & 100 & 8,581 & 53,859 & 2.0 & 207.6 & 183.9 & 177.0 & 2.1 \\
\hline $2002-2003$ & 100 & 8,544 & 52,789 & 1.9 & 203.5 & 180.1 & 173.3 & 1.5 \\
\hline 2001-2002 & 99 & 8,337 & 51,806 & 4.1 & 199.7 & 177.5 & 170.8 & 2.7 \\
\hline $2000-2001$ & 99 & 8,127 & 49,753 & 3.7 & 191.8 & 172.8 & 166.3 & 3.7 \\
\hline $1999-2000$ & 98 & 7,858 & 48,000 & 4.1 & 185.0 & 166.7 & 160.4 & 2.1 \\
\hline 1998-1999 & 97 & 7,671 & 46,130 & 3.6 & 177.8 & 163.2 & 157.1 & 1.7 \\
\hline 1997-1998 & 97 & 7,682 & 44,544 & 3.4 & 171.7 & 160.5 & 154.5 & 2.2 \\
\hline 1996-1997 & 96 & 7,562 & 43,084 & 3.4 & 166.1 & 157.0 & 151.1 & 3.0 \\
\hline 1995-1996 & 95 & 7,435 & 41,651 & 2.7 & 160.5 & 152.5 & 146.8 & 2.8 \\
\hline 1994-1995 & 95 & 7,401 & 40,573 & 3.4 & 156.4 & 148.4 & 142.8 & 2.8 \\
\hline 1993-1994 & 95 & 7,390 & 39,257 & 3.0 & 151.3 & 144.4 & 139.0 & 2.8 \\
\hline 1992-1993 & 95 & 7,375 & 38,124 & 3.0 & 146.9 & 140.5 & 134.9 & 3.2 \\
\hline 1991-1992 & 94 & 7,408 & 37,009 & 3.5 & 142.6 & 136.2 & 131.1 & 4.4 \\
\hline 1990-1991 & 94 & 7,543 & 35,761 & 5.2 & 137.8 & 130.4 & 125.8 & 4.8 \\
\hline 1989-1990 & 94 & 7,344 & 34,000 & 5.8 & 131.0 & 124.4 & 119.3 & 5.0 \\
\hline 1988-1989 & 94 & 7,252 & 32,149 & 5.4 & 123.9 & 118.5 & 113.9 & 4.1 \\
\hline 1987-1988 & 93 & 7,145 & 30,492 & 5.1 & 117.5 & 113.8 & 109.3 & 3.9 \\
\hline 1986-1987 & 92 & 6,886 & 29,021 & 6.5 & 111.9 & 109.5 & 105.5 & 1.6 \\
\hline 1985-1986 & 91 & 6,707 & 27,249 & 5.0 & 105.0 & 107.8 & 103.6 & 3.6 \\
\hline 1984-1985 & 91 & 6,456 & 25,946 & 6.9 & 100.0 & 104.1 & 100.0 & - \\
\hline
\end{tabular}

*Actual CPI figures have been revised from previous editions based upon changes published by the Bureau of Labor Statistics. These changes were caused by rounding; they are minute and are less than 0.3 in all cases.

† Includes medical and law libraries. 


\section{Table 4: Salary Trends in Canadian ARL University Libraries}

Salary figures for the current year are displayed in the context of previous years. Canadian salaries are presented in both US \$ and Canadian \$ denominations and the annual exchange rate used in the salary surveys is also listed. Canadian salaries are also compared to the changes in the Canadian Consumer Price Index (CPI) to show trends in the purchasing power of median Canadian salaries. CPI number changes are based on July CPI figures. The source for Canadian CPI data is "Table 5: The Consumer Price Index for Canada" published in The Daily, a Statistics Canada publication, available online at http://www.statcan.gc.ca/pub/62-001-x/2009010/t040-eng.htm.

\begin{tabular}{|c|c|c|c|c|c|c|c|c|c|}
\hline $\begin{array}{c}\text { Fiscal } \\
\text { Year }\end{array}$ & $\begin{array}{l}\text { No. of } \\
\text { Libs. }\end{array}$ & $\begin{array}{l}\text { Total } \\
\text { Staff }\end{array}$ & $\begin{array}{l}\text { Median } \\
\text { Salary } \\
\text { in US } \$^{+}\end{array}$ & $\begin{array}{c}\text { Median } \\
\text { Salary } \\
\text { Change }^{+}\end{array}$ & $\begin{array}{c}\text { Exchange } \\
\text { Rate }\end{array}$ & $\begin{array}{c}\text { Median } \\
\text { Salary } \\
\text { in Can. \$ }\end{array}$ & $\begin{array}{c}\text { Median } \\
\text { Salary } \\
\text { Change }\end{array}$ & $\begin{array}{l}\text { Can. } \\
\text { CPI }^{* *}\end{array}$ & $\begin{array}{c}\text { Can. } \\
\text { CPI } \\
\text { Change* }\end{array}$ \\
\hline 2009-2010 & 15 & 1,091 & $\$ 69,130$ & $-11.3 \%$ & 1.1667 & $\$ 80,654$ & $2.4 \%$ & 114.7 & $-0.9 \%$ \\
\hline 2008-2009 & 14 & 990 & 77,954 & 15.8 & 1.0101 & 78,742 & 3.3 & 115.8 & 3.4 \\
\hline $2007-2008$ & 14 & 957 & 67,331 & 6.7 & 1.1323 & 76,239 & 3.9 & 112.0 & 2.2 \\
\hline 2006-2007 & 14 & 958 & 63,112 & 11.8 & 1.16289 & 73,392 & 4.0 & 109.6 & 2.3 \\
\hline 2005-2006 & 14 & 955 & 56,474 & 7.1 & 1.24971 & 70,576 & -0.3 & 107.1 & 2.0 \\
\hline 2004-2005 & 14 & 906 & 52,707 & 16.3 & 1.34328 & 70,800 & 3.5 & 105.0 & 2.3 \\
\hline 2003-2004 & 14 & 911 & 45,310 & 6.2 & 1.51023 & 68,429 & 2.3 & 102.6 & 2.1 \\
\hline 2002-2003 & 14 & 925 & 42,657 & -0.6 & 1.56878 & 66,919 & 2.6 & 100.5 & 2.1 \\
\hline 2001-2002 & 14 & 861 & 42,928 & -1.1 & 1.51919 & 65,215 & 2.1 & 98.4 & 2.7 \\
\hline $2000-2001$ & 13 & 755 & 43,394 & 5.0 & 1.47192 & 63,873 & 2.4 & 95.8 & 2.9 \\
\hline 1999-2000 & 13 & 737 & 41,316 & -3.8 & 1.5103 & 62,400 & 2.4 & 93.1 & 1.9 \\
\hline 1998-1999 & 13 & 729 & 42,963 & -2.7 & 1.4177 & 60,909 & 0.9 & 91.4 & 1.0 \\
\hline 1997-1998 & 13 & 732 & 44,167 & 1.4 & 1.3663 & 60,346 & 1.7 & 90.5 & 1.7 \\
\hline 1996-1997 & 13 & 764 & 43,569 & 0.9 & 1.3613 & 59,310 & -0.4 & 89.0 & 1.3 \\
\hline 1995-1996 & 13 & 796 & 43,173 & -1.7 & 1.3794 & 59,554 & 1.3 & 87.9 & 2.6 \\
\hline 1994-1995 & 13 & 815 & 43,919 & -6.0 & 1.3381 & 58,768 & 0.7 & 85.7 & 0.1 \\
\hline 1993-1994 & 13 & 816 & 46,744 & -4.3 & 1.2488 & 58,374 & 2.9 & 85.6 & 1.7 \\
\hline 1992-1993 & 13 & 837 & 48,820 & 2.7 & 1.1623 & 56,744 & 3.4 & 84.2 & 1.2 \\
\hline 1991-1992 & 13 & 847 & 47,519 & 5.5 & 1.1547 & 54,870 & 3.6 & 83.2 & 6.0 \\
\hline 1990-1991 & 13 & 839 & 45,023 & 15.1 & 1.1759 & 52,942 & 12.5 & 78.5 & 4.1 \\
\hline 1989-1990 & 13 & 853 & 39,117 & 12.3 & 1.2026 & 47,042 & 5.3 & 75.4 & 5.3 \\
\hline 1988-1989 & 13 & 837 & 34,826 & 11.7 & 1.2826 & 44,668 & 5.3 & 71.6 & 3.9 \\
\hline 1987-1988 & 13 & 817 & 31,178 & 10.9 & 1.3602 & 42,408 & 9.1 & 68.9 & 4.6 \\
\hline 1986-1987 & 13 & 831 & 28,123 & -1.9 & 1.3817 & 38,858 & 1.2 & 65.9 & 4.1 \\
\hline 1985-1986 & 13 & 829 & 28,666 & 1.1 & 1.3388 & 38,378 & 7.9 & 63.3 & 4.1 \\
\hline 1984-1985 & 12 & 705 & 28,346 & -0.8 & 1.2548 & 35,569 & 0.8 & 60.8 & 4.1 \\
\hline Average & & & & $3.8 \%$ & & & $3.3 \%$ & & \\
\hline
\end{tabular}

${ }^{\dagger}$ Includes medical and law libraries.

* Canadian CPI change figures have been revised from previous editions based upon changes published by The Daily (Statistics Canada). These changes were caused by rounding; they are minute and are less than 0.3 in all cases.

** Actual Canadian CPI figures have been added to this table (not available in previous editions). 


\section{ARL NoNUNIVERSITY LIBRARIES}

TABles 5-6 

Table 5: Median and Beginning Professional Salaries in ARL Nonuniversity Libraries

\begin{tabular}{|c|c|c|c|c|c|}
\hline & \multirow{2}{*}{$\begin{array}{l}\text { No. of } \\
\text { Staff }\end{array}$} & \multicolumn{2}{|c|}{ Median Salaries* } & \multicolumn{2}{|c|}{ Beginning Salaries* } \\
\hline & & FY 2008-2009 & FY 2009-2010 & FY 2008-2009 & FY 2009-2010 \\
\hline Boston Public Library & 174 & $\$ 63,736$ & $\$ 67,184$ & $\$ 39,782$ & $\$ 40,975$ \\
\hline $\begin{array}{l}\text { Canada Institute for Scientific } \\
\text { and Technical Information * + }\end{array}$ & 188 & 72,217 & 67,639 & 49,441 & 44,446 \\
\hline Center for Research Libraries ${ }^{\dagger}$ & 34 & 42,348 & 50,799 & 33,878 & 33,878 \\
\hline Library of Congress $^{+}$ & 2,633 & 96,791 & 101,416 & 48,108 & 50,408 \\
\hline National Agricultural Library ${ }^{\dagger}$ & 93 & 79,060 & 82,845 & 48,108 & 50,408 \\
\hline Library \& Archives Canada * & 107 & 62,031 & 56,601 & 49,472 & 45,141 \\
\hline National Library of Medicine ${ }^{+}$ & 209 & 86,046 & 90,154 & 48,108 & 50,408 \\
\hline New York Public Library & 258 & 59,963 & 63,696 & 39,295 & 42,638 \\
\hline New York State Library & 57 & 58,849 & 64,305 & 47,556 & 49,968 \\
\hline Smithsonian Institution Libraries & 58 & 81,394 & 85,281 & 49,712 & 52,089 \\
\hline
\end{tabular}

* Canadian salaries are expressed in US dollars.

${ }^{+}$See footnotes. 


\section{TAble 6: Salary Trends in ARL NonUniversity Libraries}

Salary figures for the current year are displayed in the context of the previous years and compared to the changes in the Consumer Price Index (CPI) to show trends in the purchasing power of median and beginning professional salaries. Salary figures and CPI numbers have been converted to adjusted indexes, using July 1984 as the base. Actual CPI data retrieved from the US Department of Labor, Bureau of Labor Statistics' Consumer Price Index-All Urban Consumers (US All items, 1982-1984=100 - CUUR0000SA0) available online at http://www. bls.gov/data/.

\begin{tabular}{|c|c|c|c|c|c|c|c|c|}
\hline $\begin{array}{l}\text { Fiscal } \\
\text { Year }\end{array}$ & $\begin{array}{l}\text { Number of } \\
\text { Libraries }\end{array}$ & $\begin{array}{l}\text { Total } \\
\text { Staff }\end{array}$ & $\begin{array}{l}\text { Median } \\
\text { Salary* }^{*}\end{array}$ & $\begin{array}{c}\text { BPS }^{+} \\
\text {Median }\end{array}$ & $\begin{array}{c}\text { Median } \\
\text { Salary Index }\end{array}$ & $\begin{array}{l}\text { BPS }^{+} \\
\text {Index }\end{array}$ & $\begin{array}{c}\text { Actual } \\
\text { CPI }\end{array}$ & $\begin{array}{l}\text { Adjusted } \\
\text { CPI }\end{array}$ \\
\hline 2009-2010 & 10 & 3,811 & $\$ 85,229$ & $\$ 47,554$ & 251.6 & 288.1 & 215.4 & 207.3 \\
\hline 2008-2009 & 10 & 3,748 & 85,320 & 48,108 & 251.8 & 291.4 & 219.9 & 211.6 \\
\hline $2007-2008$ & 10 & 3,797 & 80,261 & 44,359 & 236.9 & 268.7 & 208.3 & 200.5 \\
\hline $2006-2007$ & 10 & 3,832 & 80,124 & 42,765 & 236.5 & 259.1 & 203.5 & 195.9 \\
\hline 2005-2006 & 10 & 3,921 & 76,083 & 38,673 & 224.6 & 234.3 & 195.4 & 188.1 \\
\hline 2004-2005 & 10 & 3,946 & 74,022 & 34,764 & 218.5 & 210.6 & 189.4 & 182.3 \\
\hline 2003-2004 & 10 & 3,877 & 70,020 & 34,739 & 206.8 & 210.4 & 183.9 & 177.0 \\
\hline $2002-2003$ & 10 & 3,804 & 65,289 & 34,739 & 192.7 & 210.4 & 180.1 & 173.3 \\
\hline 2001-2002 & 10 & 3,717 & 65,025 & 34,389 & 191.9 & 208.3 & 177.5 & 170.8 \\
\hline $2000-2001$ & 10 & 3,731 & 62,521 & 31,774 & 184.5 & 192.5 & 172.8 & 166.3 \\
\hline 1999-2000 & 10 & 3,737 & 59,916 & 30,849 & 176.8 & 186.9 & 166.7 & 160.4 \\
\hline 1998-1999 & 11 & 3,819 & 56,000 & 29,877 & 165.3 & 181.0 & 163.2 & 157.1 \\
\hline 1997-1998 & 11 & 3,779 & 55,055 & 28,724 & 162.5 & 174.0 & 160.5 & 154.5 \\
\hline 1996-1997 & 11 & 3,799 & 51,150 & 28,380 & 151.0 & 172.0 & 157.0 & 151.1 \\
\hline 1995-1996 & 11 & 3,915 & 49,149 & 28,162 & 145.1 & 170.7 & 152.5 & 146.8 \\
\hline 1994-1995 & 11 & 3,837 & 47,997 & 27,813 & 141.7 & 168.6 & 148.4 & 142.8 \\
\hline 1993-1994 & 11 & 4,003 & 44,949 & 26,806 & 132.7 & 162.5 & 144.4 & 139.0 \\
\hline 1992-1993 & 11 & 4,172 & 43,876 & 23,500 & 129.6 & 142.4 & 140.2 & 134.9 \\
\hline 1991-1992 & 11 & 2,906 & 42,455 & 23,500 & 125.4 & 142.4 & 136.2 & 131.1 \\
\hline 1990-1991 & 12 & 1,363 & 36,013 & 20,800 & 106.3 & 126.1 & 130.7 & 125.8 \\
\hline 1989-1990 & 11 & 3,767 & 40,106 & 20,195 & 118.4 & 122.4 & 124.0 & 119.3 \\
\hline 1988-1989 & 11 & 3,781 & 37,544 & 19,100 & 110.9 & 115.8 & 118.3 & 113.9 \\
\hline 1987-1988 & 11 & 3,765 & 36,250 & 18,405 & 107.0 & 111.5 & 113.6 & 109.3 \\
\hline 1986-1987 & 10 & 2,790 & 33,020 & 17,912 & 97.5 & 108.6 & 109.6 & 105.5 \\
\hline 1985-1986 & 12 & 3,874 & 33,720 & 17,308 & 99.6 & 104.9 & 107.6 & 103.6 \\
\hline 1984-1985 & 11 & 3,840 & 33,863 & 16,500 & 100.0 & 100.0 & 103.9 & 100.0 \\
\hline
\end{tabular}




\title{
ARL UNIVERSITY LIBRARIES
}

\author{
TABLes 7-25
}


Table 7: Filled Positions; Average, Median, Beginning Professional Salaries; and Average YeARS OF EXPERIENCE IN ARL UNIVERSITY LibRARIES, FY 2009-2010

\begin{tabular}{|c|c|c|c|c|c|c|c|c|}
\hline \multirow[b]{2}{*}{ Institution } & \multirow{2}{*}{$\begin{array}{c}\text { Filled } \\
\text { Positions } \\
\text { FY } 2010\end{array}$} & \multicolumn{2}{|c|}{$\begin{array}{l}\text { Average } \\
\text { Salaries }\end{array}$} & \multicolumn{2}{|c|}{$\begin{array}{l}\text { Median } \\
\text { Salaries }\end{array}$} & \multicolumn{2}{|c|}{$\begin{array}{c}\text { Beginning } \\
\text { Salaries }\end{array}$} & \multirow{2}{*}{$\begin{array}{l}\text { Average } \\
\text { Yrs. Exp. } \\
\text { FY } 2010\end{array}$} \\
\hline & & FY 2009 & FY 2010 & FY 2009 & FY 2010 & FY 2009 & FY 2010 & \\
\hline Alabama $^{\neq}$ & 60 & $\$ 58,655$ & $\$ 58,272$ & $\$ 52,205$ & $\$ 52,204$ & $\$ 42,000$ & $\$ 42,000$ & 15.2 \\
\hline Alberta $^{+\neq}$ & 62 & 89,260 & 84,172 & 95,438 & 89,319 & 50,126 & 43,398 & 17.5 \\
\hline Arizona ${ }^{\ddagger}$ & 60 & 66,447 & 65,897 & 61,595 & 59,915 & 47,093 & 48,605 & 17.6 \\
\hline Arizona State ${ }^{\ddagger}$ & 58 & 63,110 & 66,091 & 61,000 & 65,071 & 43,000 & 43,000 & 18.8 \\
\hline Auburn ${ }^{\ddagger}$ & 45 & 60,231 & 58,382 & 55,290 & 53,705 & 44,720 & 44,720 & 15.1 \\
\hline Boston University ${ }^{\ddagger}$ & 59 & 59,236 & 57,615 & 55,200 & 56,600 & 42,300 & 43,700 & 16.5 \\
\hline Boston College $^{\ddagger}$ & 61 & 69,672 & 69,739 & 67,184 & 67,280 & 42,300 & 42,300 & 19.1 \\
\hline Brigham Young ${ }^{\ddagger}$ & 111 & 63,925 & 66,005 & 62,400 & 63,950 & 48,000 & 51,000 & 18.2 \\
\hline British Columbia $^{+\neq}$ & 94 & 79,910 & 73,023 & 80,769 & 70,005 & 53,021 & 47,429 & 15.0 \\
\hline Brown ${ }^{\ddagger}$ & 74 & 67,804 & 65,880 & 63,854 & 61,549 & 39,500 & 39,500 & 20.5 \\
\hline Calgary $^{\ddagger}$ & 57 & $\mathrm{~N} / \mathrm{A}$ & 81,275 & N/A & 76,749 & $\mathrm{~N} / \mathrm{A}$ & 49,713 & 18.3 \\
\hline California, Berkeley ${ }^{\ddagger}$ & 113 & 82,952 & 83,853 & 80,374 & 82,524 & 46,164 & 47,087 & 18.2 \\
\hline California, Davis $^{\ddagger}$ & 43 & 79,652 & 81,135 & 82,637 & 88,488 & 46,164 & 46,164 & 21.1 \\
\hline California, Irvine & 56 & 76,045 & 76,645 & 75,708 & 75,708 & 46,164 & 46,164 & 15.4 \\
\hline California, Los Angeles ${ }^{\ddagger}$ & 147 & 75,812 & 78,189 & 73,799 & 75,708 & 46,164 & 46,164 & 16.1 \\
\hline California, Riverside ${ }^{\ddagger}$ & 45 & 74,166 & 77,661 & 74,400 & 75,708 & 47,087 & 47,087 & 21.0 \\
\hline California, San Diego ${ }^{*}$ & 93 & 76,016 & 77,158 & 75,708 & 75,708 & 46,164 & 46,164 & 16.4 \\
\hline California, Santa Barbara ${ }^{\ddagger}$ & 61 & 71,466 & 71,378 & 68,892 & 68,892 & 46,164 & 46,164 & 17.0 \\
\hline Case Western Reserve ${ }^{\ddagger}$ & 41 & 57,972 & 58,788 & 54,389 & 55,227 & 35,000 & 35,000 & 15.3 \\
\hline Chicago $^{\ddagger}$ & 72 & 74,480 & 76,254 & 70,290 & 72,329 & 48,204 & 48,204 & 19.3 \\
\hline Cincinnati ${ }^{\ddagger}$ & 52 & 66,847 & 65,445 & 61,332 & 61,879 & 39,000 & 40,000 & 20.9 \\
\hline Colorado $^{\ddagger}$ & 41 & 66,826 & 67,523 & 64,388 & 64,573 & 44,000 & 44,000 & 16.4 \\
\hline Colorado State & 46 & 68,355 & 68,311 & 65,300 & 65,300 & 45,400 & 45,000 & 17.6 \\
\hline Columbia & 185 & 72,352 & 72,939 & 64,265 & 64,385 & 51,500 & 51,500 & 15.8 \\
\hline Connecticut $^{\ddagger}$ & 58 & 79,855 & 82,083 & 78,680 & 78,079 & 48,000 & 48,000 & 18.3 \\
\hline Cornell ${ }^{\ddagger}$ & 103 & 69,564 & 71,548 & 63,600 & 64,239 & 47,000 & 47,000 & 16.1 \\
\hline Dartmouth $^{\ddagger}$ & 48 & 72,539 & 72,208 & 69,536 & 67,933 & 42,500 & 45,500 & 17.6 \\
\hline Delaware ${ }^{\ddagger}$ & 58 & 72,792 & 75,703 & 71,037 & 73,353 & 43,600 & 43,600 & 18.2 \\
\hline Duke ${ }^{\ddagger}$ & 122 & 63,058 & 64,870 & 58,583 & 60,000 & 44,000 & 45,000 & 16.8 \\
\hline Emory ${ }^{\neq}$ & 73 & 69,025 & 68,747 & 63,653 & 63,068 & 41,500 & 42,000 & 15.1 \\
\hline Florida ${ }^{\ddagger}$ & 68 & 58,974 & 60,450 & 55,045 & 56,388 & 42,000 & 42,000 & 15.4 \\
\hline Florida State $^{\ddagger}$ & 48 & 49,803 & 54,858 & 47,000 & 49,805 & 42,000 & 42,000 & 13.9 \\
\hline George Washington & 39 & 70,759 & 74,353 & 65,328 & 67,143 & 47,000 & 47,000 & 15.6 \\
\hline Georgetown ${ }^{\ddagger}$ & 53 & 69,739 & 69,227 & 63,538 & 63,538 & 45,000 & 45,000 & 21.4 \\
\hline Georgia ${ }^{*}$ & 74 & 57,628 & 56,544 & 51,005 & 50,000 & 38,000 & 38,000 & 16.0 \\
\hline Georgia Tech & 42 & 60,944 & 63,526 & 56,270 & 58,016 & 42,000 & 44,000 & 16.7 \\
\hline Guelph $^{+\ddagger}$ & 50 & 77,319 & 69,113 & 71,928 & 66,902 & 57,242 & 51,169 & 17.7 \\
\hline Harvard ${ }^{\ddagger}$ & 454 & 78,019 & 77,319 & 70,900 & 70,720 & 48,800 & 48,800 & 15.8 \\
\hline Hawaii ${ }^{\ddagger}$ & 83 & 67,491 & 66,721 & 67,391 & 66,225 & 42,649 & 42,660 & 16.0 \\
\hline Houston ${ }^{\ddagger}$ & 44 & 63,512 & 61,669 & 62,480 & 56,563 & 42,000 & 43,000 & 14.1 \\
\hline Howard ${ }^{\ddagger}$ & 30 & 53,741 & 43,547 & 53,440 & 43,010 & 36,000 & 38,000 & 18.9 \\
\hline Illinois, Chicago ${ }^{\ddagger}$ & 47 & 61,206 & 62,208 & 56,457 & 56,555 & 47,000 & 47,000 & 17.6 \\
\hline Illinois, Urbana & 151 & 67,213 & 66,755 & 62,036 & 61,645 & 46,000 & 46,000 & 14.4 \\
\hline Indiana ${ }^{\neq}$ & 80 & 63,786 & 63,870 & 60,011 & 59,331 & 40,400 & 40,400 & 19.1 \\
\hline Iowa $^{\ddagger}$ & 71 & 63,196 & 62,529 & 56,796 & 55,573 & 41,000 & 41,000 & 17.7 \\
\hline Iowa State ${ }^{\ddagger}$ & 53 & 61,863 & 63,217 & 59,887 & 61,664 & 43,000 & 43,000 & 19.7 \\
\hline Johns Hopkins ${ }^{\ddagger}$ & 75 & 67,265 & 68,926 & 63,702 & 65,690 & 50,026 & 50,026 & 17.4 \\
\hline Kansas $^{\ddagger}$ & 86 & 61,056 & 60,877 & 56,200 & 54,806 & 49,000 & 51,000 & 15.5 \\
\hline Kent State ${ }^{\ddagger}$ & 59 & 57,317 & 63,997 & 53,777 & 60,301 & 43,389 & 55,367 & 15.7 \\
\hline Kentucky ${ }^{\ddagger}$ & 65 & 60,909 & 60,966 & 59,697 & 59,839 & 41,000 & 41,000 & 21.1 \\
\hline $\mathrm{Laval}^{+}$ & 62 & 68,511 & 61,963 & 69,901 & 62,474 & 47,613 & 42,468 & 14.4 \\
\hline Louisiana State & 50 & 51,839 & 50,821 & 47,715 & 46,462 & 38,000 & 38,000 & 13.5 \\
\hline Louisville ${ }^{\ddagger}$ & 31 & 61,851 & 59,483 & 56,845 & 55,884 & 37,000 & 37,000 & 18.2 \\
\hline $\mathrm{McGill}^{+}$ & 65 & 73,148 & 63,797 & 67,233 & 57,834 & 49,500 & 42,856 & 16.7 \\
\hline McMaster $^{+\ddagger}$ & 48 & 78,776 & 61,608 & 78,858 & 62,508 & 44,771 & 40,128 & 16.8 \\
\hline Manitoba $^{+\neq}$ & 41 & 89,270 & 81,855 & 93,351 & 85,771 & 46,970 & 41,845 & 22.8 \\
\hline Maryland & 84 & 67,924 & 68,874 & 66,419 & 66,603 & 42,000 & 40,000 & 19.9 \\
\hline Massachusetts ${ }^{\ddagger}$ & 59 & 70,508 & 69,882 & 72,263 & 72,263 & 42,155 & 42,155 & 16.4 \\
\hline $\mathrm{MIT}^{\ddagger}$ & 94 & 75,733 & 74,430 & 70,900 & 70,959 & 51,000 & 51,750 & 16.0 \\
\hline
\end{tabular}


Table 7: Filled Positions; Average, Median, Beginning Professional Salaries; and Average YeARS OF EXPERIENCE IN ARL UNIVERSITY LibRARIES, FY 2009-2010

\begin{tabular}{|c|c|c|c|c|c|c|c|c|}
\hline \multirow[b]{2}{*}{ Institution } & \multirow{2}{*}{$\begin{array}{c}\text { Filled } \\
\text { Positions } \\
\text { FY } 2010\end{array}$} & \multicolumn{2}{|c|}{$\begin{array}{l}\text { Average } \\
\text { Salaries }\end{array}$} & \multicolumn{2}{|c|}{$\begin{array}{l}\text { Median } \\
\text { Salaries }\end{array}$} & \multicolumn{2}{|c|}{$\begin{array}{c}\text { Beginning } \\
\text { Salaries }\end{array}$} & \multirow{2}{*}{$\begin{array}{l}\text { Average } \\
\text { Yrs. Exp. } \\
\text { FY } 2010\end{array}$} \\
\hline & & FY 2009 & FY 2010 & FY 2009 & FY 2010 & FY 2009 & FY 2010 & \\
\hline Miami $^{\ddagger}$ & 49 & 69,165 & 68,278 & 64,935 & 61,728 & 45,000 & 45,000 & 16.5 \\
\hline Michigan ${ }^{\ddagger}$ & 122 & 70,031 & 67,767 & 65,564 & 63,717 & 42,000 & 42,000 & 16.0 \\
\hline Michigan State ${ }^{\ddagger}$ & 67 & 63,867 & 68,894 & 60,000 & 65,850 & 47,000 & 47,000 & 16.4 \\
\hline Minnesota & 107 & 67,975 & 66,794 & 65,446 & 63,557 & 40,000 & 42,000 & 16.4 \\
\hline Missouri ${ }^{\ddagger}$ & 39 & 55,264 & 59,914 & 48,854 & 55,964 & 40,000 & 40,000 & 19.4 \\
\hline Montreal ${ }^{\dagger \neq}$ & 92 & 69,342 & 62,740 & 66,631 & 59,349 & 41,738 & 36,968 & 15.1 \\
\hline Nebraska & 47 & 65,751 & 65,057 & 57,671 & 57,472 & 50,000 & 50,000 & 19.4 \\
\hline New Mexico ${ }^{\ddagger}$ & 43 & 78,591 & 72,938 & 76,307 & 68,853 & 40,000 & 40,000 & 20.8 \\
\hline New York University ${ }^{\ddagger}$ & 53 & 78,920 & 79,018 & 70,769 & 69,989 & 52,000 & 52,000 & 18.4 \\
\hline North Carolina & 99 & 67,177 & 67,637 & 63,750 & 63,712 & 44,000 & 44,000 & 17.9 \\
\hline North Carolina State ${ }^{\ddagger}$ & 84 & 70,667 & 73,019 & 64,895 & 65,000 & 49,000 & 50,000 & 11.0 \\
\hline Northwestern ${ }^{\ddagger}$ & 88 & 66,328 & 67,873 & 62,847 & 63,429 & 42,000 & 43,000 & 17.0 \\
\hline Notre Dame ${ }^{\ddagger}$ & 63 & 68,224 & 69,492 & 65,738 & 66,508 & 40,000 & 41,200 & 18.3 \\
\hline Ohio University ${ }^{\ddagger}$ & 47 & 54,210 & 55,522 & 48,170 & 48,787 & 41,500 & 41,000 & 13.6 \\
\hline Ohio State $^{\ddagger}$ & 122 & 57,439 & 59,476 & 52,432 & 54,450 & 43,000 & 46,000 & 14.4 \\
\hline Oklahoma & 41 & 54,228 & 55,853 & 52,228 & 53,742 & 42,000 & 42,000 & 14.4 \\
\hline Oklahoma State ${ }^{\ddagger}$ & 65 & 56,703 & 56,580 & 53,640 & 52,657 & 36,000 & 38,000 & 16.8 \\
\hline Oregon ${ }^{\ddagger}$ & 57 & 56,965 & 59,782 & 53,792 & 55,973 & 40,000 & 40,000 & 16.2 \\
\hline Pennsylvania ${ }^{\ddagger}$ & 105 & 65,914 & 65,997 & 61,122 & 61,850 & 42,500 & 43,500 & 16.1 \\
\hline Pennsylvania State ${ }^{\ddagger}$ & 145 & 69,421 & 67,897 & 65,556 & 64,224 & 42,436 & 42,436 & 19.3 \\
\hline Pittsburgh ${ }^{\ddagger}$ & 65 & 66,544 & 66,551 & 59,984 & 60,711 & 34,000 & 34,000 & 18.8 \\
\hline Princeton ${ }^{\neq}$ & 122 & 77,939 & 79,015 & 72,150 & 73,799 & 50,000 & 63,200 & 21.0 \\
\hline Purdue ${ }^{\ddagger}$ & 65 & 66,957 & 65,573 & 61,000 & 60,513 & 45,000 & 47,000 & 18.5 \\
\hline Queen`s $^{+}$ & 35 & 88,107 & 80,504 & 89,828 & 83,438 & 49,500 & 44,227 & 20.0 \\
\hline Rice & 59 & 61,433 & 62,215 & 55,450 & 55,833 & 38,300 & 37,200 & 15.9 \\
\hline Rochester $^{\ddagger}$ & 74 & 55,965 & 55,837 & 52,540 & 53,000 & 38,168 & 38,168 & 14.8 \\
\hline Rutgers $^{\ddagger}$ & 84 & 88,899 & 89,058 & 92,106 & 91,751 & 47,390 & 49,286 & 21.7 \\
\hline Saskatchewan ${ }^{+\ddagger}$ & 40 & 85,401 & 76,307 & 85,977 & 78,850 & 49,600 & 45,197 & 17.4 \\
\hline South Carolina ${ }^{\ddagger}$ & 53 & 53,670 & 53,658 & 49,838 & 49,838 & 34,000 & 34,000 & 16.6 \\
\hline Southern California ${ }^{\ddagger}$ & 103 & 73,788 & 74,479 & 66,944 & 67,650 & 48,500 & 48,500 & 18.4 \\
\hline Southern Illinois & 38 & 57,003 & 58,264 & 49,117 & 52,536 & 43,000 & 44,000 & 14.0 \\
\hline SUNY Albany ${ }^{\ddagger}$ & 62 & 67,251 & 60,964 & 59,704 & 58,822 & 38,500 & 39,000 & 16.5 \\
\hline SUNY Buffalo ₹ & 76 & 69,982 & 72,916 & 66,684 & 69,048 & 45,000 & 45,000 & 20.3 \\
\hline SUNY Stony Brook & 26 & 75,948 & 79,837 & 73,259 & 75,457 & 44,000 & 42,000 & 22.2 \\
\hline Syracuse ${ }^{\ddagger}$ & 54 & 65,441 & 65,847 & 61,493 & 60,648 & N/A & None & 18.2 \\
\hline Temple ${ }^{\ddagger}$ & 40 & 66,114 & 66,849 & 56,020 & 58,140 & 42,744 & 44,044 & 19.1 \\
\hline Tennessee $^{\ddagger}$ & 42 & 70,344 & 69,380 & 67,823 & 66,307 & 44,000 & 44,000 & 18.5 \\
\hline Texas $^{\ddagger}$ & 120 & 66,554 & 66,882 & 58,958 & 59,260 & 45,000 & 46,000 & 16.7 \\
\hline Texas A\&M ${ }^{\ddagger}$ & 134 & 59,250 & 62,441 & 54,156 & 55,704 & 47,000 & 47,500 & 16.0 \\
\hline Texas Tech ${ }^{\ddagger}$ & 61 & 60,494 & 60,690 & 55,036 & 57,135 & 45,000 & 45,000 & 11.8 \\
\hline Toronto ${ }^{\dagger \ddagger}$ & 143 & 90,829 & 78,211 & 91,773 & 75,194 & 51,381 & 44,484 & 15.7 \\
\hline Tulane & 33 & 62,896 & 60,534 & 60,000 & 56,325 & 40,000 & 40,000 & 16.5 \\
\hline Utah $^{\ddagger}$ & 54 & 61,119 & 61,470 & 57,250 & 56,312 & 44,000 & 44,000 & 19.0 \\
\hline Vanderbilt ${ }^{\ddagger}$ & 62 & 59,810 & 58,991 & 54,666 & 54,655 & 40,000 & 40,500 & 17.4 \\
\hline Virginia $^{\ddagger}$ & 74 & 69,246 & 67,988 & 60,450 & 60,000 & 44,000 & 44,000 & 15.9 \\
\hline Virginia Tech & 36 & 65,516 & 63,148 & 62,556 & 58,540 & 41,000 & 40,000 & 16.7 \\
\hline Washington ${ }^{\ddagger}$ & 117 & 66,517 & 66,476 & 60,312 & 59,934 & 42,600 & 42,600 & 19.7 \\
\hline Washington State ${ }^{\ddagger}$ & 44 & 59,820 & 62,755 & 56,482 & 58,172 & 38,500 & 38,500 & 18.7 \\
\hline Washington U.-St. Louis ${ }^{\ddagger}$ & 63 & 59,497 & 59,037 & 54,468 & 53,740 & 40,000 & 40,000 & 16.1 \\
\hline Waterloo $^{+\neq}$ & 36 & 77,432 & 70,230 & 78,311 & 70,260 & 49,090 & 44,402 & 17.9 \\
\hline Wayne State ${ }^{\ddagger}$ & 67 & 54,962 & 61,261 & 50,031 & 54,705 & 40,500 & 40,500 & 15.0 \\
\hline Western Ontario ${ }^{+\neq}$ & 68 & 68,168 & 59,073 & 63,872 & 55,725 & 46,906 & 40,610 & 13.2 \\
\hline Wisconsin $^{\ddagger}$ & 157 & 60,304 & 61,856 & 57,808 & 58,654 & 40,526 & 40,526 & 16.5 \\
\hline Yale ${ }^{\ddagger}$ & 213 & 82,622 & 81,088 & 76,974 & 76,300 & 50,500 & 50,500 & 18.9 \\
\hline York $^{+\neq}$ & 54 & 95,755 & 82,297 & 88,458 & 77,321 & 48,510 & 41,999 & 17.2 \\
\hline
\end{tabular}

Directors are included in figures for average years of experience and filled positions, but not in either the average or median salary statistics. Excludes medical and law libraries. See Tables 35 and 42 for statistics related to medical and law library salaries.

+ Canadian salaries are expressed in US dollars. ₹ See Footnotes. 


\section{Table 8: Beginning Professional Salaries in ARL University Libraries RANK ORder TABLE, FY 2008-2009}

\begin{tabular}{|c|c|c|c|c|c|}
\hline Rank & Institution & Salary & Rank & Institution & Salary \\
\hline 1 & Guelph & 57,242 & 58 & Delaware & 43,600 \\
\hline 2 & British Columbia & 53,021 & 59 & Kent State & 43,389 \\
\hline 3 & New York & 52,000 & 60 & Arizona State & 43,000 \\
\hline 4 & Columbia & 51,500 & 60 & Iowa State & 43,000 \\
\hline 5 & Toronto & 51,381 & 60 & Ohio State & 43,000 \\
\hline 6 & MIT & 51,000 & 60 & Southern Illinois & 43,000 \\
\hline 7 & Yale & 50,500 & 64 & Temple & 42,744 \\
\hline 8 & Alberta & 50,126 & 65 & Hawaii & 42,649 \\
\hline 9 & Johns Hopkins & 50,026 & 66 & Washington & 42,600 \\
\hline 10 & Nebraska & 50,000 & 67 & Dartmouth & 42,500 \\
\hline 10 & Princeton & 50,000 & 67 & Pennsylvania & 42,500 \\
\hline 12 & Saskatchewan & 49,600 & 69 & Pennsylvania State & 42,436 \\
\hline 13 & McGill & 49,500 & 70 & Boston University & 42,300 \\
\hline 13 & Queen's & 49,500 & 70 & Boston College & 42,300 \\
\hline 15 & Waterloo & 49,090 & 72 & Massachusetts & 42,155 \\
\hline 16 & Kansas & 49,000 & 73 & Alabama & 42,000 \\
\hline 16 & North Carolina State & 49,000 & 73 & Florida & 42,000 \\
\hline 18 & Harvard & 48,800 & 73 & Florida State & 42,000 \\
\hline 19 & York & 48,510 & 73 & Georgia Tech & 42,000 \\
\hline 20 & Southern California & 48,500 & 73 & Houston & 42,000 \\
\hline 21 & Chicago & 48,204 & 73 & Maryland & 42,000 \\
\hline 22 & Brigham Young & 48,000 & 73 & Michigan & 42,000 \\
\hline 22 & Connecticut & 48,000 & 73 & Northwestern & 42,000 \\
\hline 24 & Laval & 47,613 & 73 & Oklahoma & 42,000 \\
\hline 25 & Rutgers & 47,390 & 82 & Montreal & 41,738 \\
\hline 26 & Arizona & 47,093 & 83 & Emory & 41,500 \\
\hline 27 & Calif. Riverside & 47,087 & 83 & Ohio & 41,500 \\
\hline 28 & Cornell & 47,000 & 85 & Iowa & 41,000 \\
\hline 28 & George Washington & 47,000 & 85 & Kentucky & 41,000 \\
\hline 28 & Illinois, Chicago & 47,000 & 85 & Virginia Tech & 41,000 \\
\hline 28 & Michigan State & 47,000 & 88 & Wisconsin & 40,526 \\
\hline 28 & Texas A\&M & 47,000 & 89 & Wayne State & 40,500 \\
\hline 33 & Manitoba & 46,970 & 90 & Indiana & 40,400 \\
\hline 34 & Western Ontario & 46,906 & 91 & Minnesota & 40,000 \\
\hline 35 & Calif. Berkeley & 46,164 & 91 & Missouri & 40,000 \\
\hline 35 & Calif. Davis & 46,164 & 91 & New Mexico & 40,000 \\
\hline 35 & Calif. Irvine & 46,164 & 91 & Notre Dame & 40,000 \\
\hline 35 & Calif. Los Angeles & 46,164 & 91 & Oregon & 40,000 \\
\hline 35 & Calif. San Diego & 46,164 & 91 & Tulane & 40,000 \\
\hline 35 & Calif. Santa Barbara & 46,164 & 91 & Vanderbilt & 40,000 \\
\hline 41 & Illinois, Urbana & 46,000 & 91 & Washington-St. Louis & 40,000 \\
\hline 42 & Colorado State & 45,400 & 99 & Brown & 39,500 \\
\hline 43 & Georgetown & 45,000 & 100 & Cincinnati & 39,000 \\
\hline 43 & Miami & 45,000 & 101 & SUNY Albany & 38,500 \\
\hline 43 & Purdue & 45,000 & 101 & Washington State & 38,500 \\
\hline 43 & SUNY Buffalo & 45,000 & 103 & Rice & 38,300 \\
\hline 43 & Texas & 45,000 & 104 & Rochester & 38,168 \\
\hline 43 & Texas Tech & 45,000 & 105 & Georgia & 38,000 \\
\hline 49 & McMaster & 44,771 & 105 & Louisiana State & 38,000 \\
\hline 50 & Auburn & 44,720 & 107 & Louisville & 37,000 \\
\hline 51 & Colorado & 44,000 & 108 & Howard & 36,000 \\
\hline 51 & Duke & 44,000 & 108 & Oklahoma State & 36,000 \\
\hline 51 & North Carolina & 44,000 & 110 & Case Western Reserve & 35,000 \\
\hline 51 & SUNY Stony Brook & 44,000 & 111 & Pittsburgh & 34,000 \\
\hline 51 & Tennessee & 44,000 & 111 & South Carolina & 34,000 \\
\hline 51 & Utah & 44,000 & 113 & Syracuse & $\mathrm{N} / \mathrm{A}$ \\
\hline 51 & Virginia & 44,000 & & & \\
\hline
\end{tabular}

Reprinted from ARL Annual Salary Survey 2008-2009. Beginning salary figures represent officially designated base, not necessarily salaries of actual incumbents.

Excludes medical and law libraries. See Tables 36 and 43 for statistics related to medical and law library salaries.

Canadian salaries are expressed in US dollars. 


\section{Table 9: Beginning Professional Salaries in ARL University Libraries RANK ORder TABLE, FY 2009-2010}

\begin{tabular}{|c|c|c|c|c|c|}
\hline Rank & Institution & Salary & Rank & Institution & Salary \\
\hline 1 & Princeton & 63,200 & 58 & Delaware & 43,600 \\
\hline 2 & Kent State & 55,367 & 59 & Pennsylvania & 43,500 \\
\hline 3 & New York & 52,000 & 60 & Alberta & 43,398 \\
\hline 4 & MIT & 51,750 & 61 & Arizona State & 43,000 \\
\hline 5 & Columbia & 51,500 & 61 & Houston & 43,000 \\
\hline 6 & Guelph & 51,169 & 61 & Iowa State & 43,000 \\
\hline 7 & Brigham Young & 51,000 & 61 & Northwestern & 43,000 \\
\hline 7 & Kansas & 51,000 & 65 & McGill & 42,856 \\
\hline 9 & Yale & 50,500 & 66 & Hawaii & 42,660 \\
\hline 10 & Johns Hopkins & 50,026 & 67 & Washington & 42,600 \\
\hline 11 & Nebraska & 50,000 & 68 & Laval & 42,468 \\
\hline 11 & North Carolina State & 50,000 & 69 & Pennsylvania State & 42,436 \\
\hline 13 & Calgary & 49,713 & 70 & Boston College & 42,300 \\
\hline 14 & Rutgers & 49,286 & 71 & Massachusetts & 42,155 \\
\hline 15 & Harvard & 48,800 & 72 & Alabama & 42,000 \\
\hline 16 & Arizona & 48,605 & 72 & Emory & 42,000 \\
\hline 17 & Southern California & 48,500 & 72 & Florida & 42,000 \\
\hline 18 & Chicago & 48,204 & 72 & Florida State & 42,000 \\
\hline 19 & Connecticut & 48,000 & 72 & Michigan & 42,000 \\
\hline 20 & Texas A\&M & 47,500 & 72 & Minnesota & 42,000 \\
\hline 21 & British Columbia & 47,429 & 72 & Oklahoma & 42,000 \\
\hline 22 & Calif. Berkeley & 47,087 & 72 & SUNY Stony Brook & 42,000 \\
\hline 22 & Calif. Riverside & 47,087 & 80 & York & 41,999 \\
\hline 24 & Cornell & 47,000 & 81 & Manitoba & 41,845 \\
\hline 24 & George Washington & 47,000 & 82 & Notre Dame & 41,200 \\
\hline 24 & Illinois, Chicago & 47,000 & 83 & Iowa & 41,000 \\
\hline 24 & Michigan State & 47,000 & 83 & Kentucky & 41,000 \\
\hline 24 & Purdue & 47,000 & 83 & Ohio & 41,000 \\
\hline 29 & Calif. Davis & 46,164 & 86 & Western Ontario & 40,610 \\
\hline 29 & Calif. Irvine & 46,164 & 87 & Wisconsin & 40,526 \\
\hline 29 & Calif. Los Angeles & 46,164 & 88 & Vanderbilt & 40,500 \\
\hline 29 & Calif. San Diego & 46,164 & 88 & Wayne State & 40,500 \\
\hline 29 & Calif. Santa Barbara & 46,164 & 90 & Indiana & 40,400 \\
\hline 34 & Illinois, Urbana & 46,000 & 91 & McMaster & 40,128 \\
\hline 34 & Ohio State & 46,000 & 92 & Cincinnati & 40,000 \\
\hline 34 & Texas & 46,000 & 92 & Maryland & 40,000 \\
\hline 37 & Dartmouth & 45,500 & 92 & Missouri & 40,000 \\
\hline 38 & Saskatchewan & 45,197 & 92 & New Mexico & 40,000 \\
\hline 39 & Colorado State & 45,000 & 92 & Oregon & 40,000 \\
\hline 39 & Duke & 45,000 & 92 & Tulane & 40,000 \\
\hline 39 & Georgetown & 45,000 & 92 & Virginia Tech & 40,000 \\
\hline 39 & Miami & 45,000 & 92 & Washington-St. Louis & 40,000 \\
\hline 39 & SUNY Buffalo & 45,000 & 100 & Brown & 39,500 \\
\hline 39 & Texas Tech & 45,000 & 101 & SUNY Albany & 39,000 \\
\hline 45 & Auburn & 44,720 & 102 & Washington State & 38,500 \\
\hline 46 & Toronto & 44,484 & 103 & Rochester & 38,168 \\
\hline 47 & Waterloo & 44,402 & 104 & Georgia & 38,000 \\
\hline 48 & Queen's & 44,227 & 104 & Howard & 38,000 \\
\hline 49 & Temple & 44,044 & 104 & Louisiana State & 38,000 \\
\hline 50 & Colorado & 44,000 & 104 & Oklahoma State & 38,000 \\
\hline 50 & Georgia Tech & 44,000 & 108 & Rice & 37,200 \\
\hline 50 & North Carolina & 44,000 & 109 & Louisville & 37,000 \\
\hline 50 & Southern Illinois & 44,000 & 110 & Montreal & 36,968 \\
\hline 50 & Tennessee & 44,000 & 111 & Case Western Reserve & 35,000 \\
\hline 50 & Utah & 44,000 & 112 & Pittsburgh & 34,000 \\
\hline 50 & Virginia & 44,000 & 112 & South Carolina & 34,000 \\
\hline 57 & Boston University & 43,700 & $\mathrm{~N} / \mathrm{A}$ & Syracuse & None \\
\hline
\end{tabular}

Beginning salary figures represent officially designated base, not necessarily salaries of actual incumbents. Excludes medical and law libraries. See Tables 36 and 43 for statistics related to medical and law library salaries. Canadian salaries are expressed in US dollars. 


\section{Table 10: Median Professional Salaries in ARL University Libraries \\ RANK ORDER TABLE, FY 2008-2009}

\begin{tabular}{|c|c|c|c|c|c|}
\hline Rank & Institution & Salary & Rank & Institution & Salary \\
\hline 1 & Alberta & 95,438 & 58 & Virginia Tech & 62,556 \\
\hline 2 & Manitoba & 93,351 & 59 & Houston & 62,480 \\
\hline 3 & Rutgers & 92,106 & 60 & Brigham Young & 62,400 \\
\hline 4 & Toronto & 91,773 & 61 & Illinois, Urbana & 62,036 \\
\hline 5 & Queen`s & 89,828 & 62 & Arizona & 61,595 \\
\hline 6 & York & 88,458 & 63 & Syracuse & 61,493 \\
\hline 7 & Saskatchewan & 85,977 & 64 & Cincinnati & 61,332 \\
\hline 8 & California, Davis & 82,637 & 65 & Pennsylvania & 61,122 \\
\hline 9 & British Columbia & 80,769 & 66 & Arizona State & 61,000 \\
\hline 10 & California, Berkeley & 80,374 & 66 & Purdue & 61,000 \\
\hline 11 & McMaster & 78,858 & 68 & Virginia & 60,450 \\
\hline 12 & Connecticut & 78,680 & 69 & Washington & 60,312 \\
\hline 13 & Waterloo & 78,311 & 70 & Indiana & 60,011 \\
\hline 14 & Yale & 76,974 & 71 & Michigan State & 60,000 \\
\hline 15 & New Mexico & 76,307 & 71 & Tulane & 60,000 \\
\hline 16 & California, Irvine & 75,708 & 73 & Pittsburgh & 59,984 \\
\hline 16 & California, San Diego & 75,708 & 74 & Iowa State & 59,887 \\
\hline 18 & California, Riverside & 74,400 & 75 & SUNY Albany & 59,704 \\
\hline 19 & California, Los Angeles & 73,799 & 76 & Kentucky & 59,697 \\
\hline 20 & SUNY Stony Brook & 73,259 & 77 & Texas & 58,958 \\
\hline 21 & Massachusetts & 72,263 & 78 & Duke & 58,583 \\
\hline 22 & Princeton & 72,150 & 79 & Wisconsin & 57,808 \\
\hline 23 & Guelph & 71,928 & 80 & Nebraska & 57,671 \\
\hline 24 & Delaware & 71,037 & 81 & Utah & 57,250 \\
\hline 25 & Harvard & 70,900 & 82 & Louisville & 56,845 \\
\hline 25 & MIT & 70,900 & 83 & Iowa & 56,796 \\
\hline 27 & New York University & 70,769 & 84 & Washington State & 56,482 \\
\hline 28 & Chicago & 70,290 & 85 & Illinois, Chicago & 56,457 \\
\hline 29 & Laval & 69,901 & 86 & Georgia Tech & 56,270 \\
\hline 30 & Dartmouth & 69,536 & 87 & Kansas & 56,200 \\
\hline 31 & California, Santa Barbara & 68,892 & 88 & Temple & 56,020 \\
\hline 32 & Tennessee & 67,823 & 89 & Rice & 55,450 \\
\hline 33 & Hawaii & 67,391 & 90 & Auburn & 55,290 \\
\hline 34 & McGill & 67,233 & 91 & Boston University & 55,200 \\
\hline 35 & Boston College & 67,184 & 92 & Florida & 55,045 \\
\hline 36 & Southern California & 66,944 & 93 & Texas Tech & 55,036 \\
\hline 37 & SUNY Buffalo & 66,684 & 94 & Vanderbilt & 54,666 \\
\hline 38 & Montreal & 66,631 & 95 & Washington U.-St. Louis & 54,468 \\
\hline 39 & Maryland & 66,419 & 96 & Case Western Reserve & 54,389 \\
\hline 40 & Notre Dame & 65,738 & 97 & Texas A\&M & 54,156 \\
\hline 41 & Michigan & 65,564 & 98 & Oregon & 53,792 \\
\hline 42 & Pennsylvania State & 65,556 & 99 & Kent State & 53,777 \\
\hline 43 & Minnesota & 65,446 & 100 & Oklahoma State & 53,640 \\
\hline 44 & George Washington & 65,328 & 101 & Howard & 53,440 \\
\hline 45 & Colorado State & 65,300 & 102 & Rochester & 52,540 \\
\hline 46 & Miami & 64,935 & 103 & Ohio State & 52,432 \\
\hline 47 & North Carolina State & 64,895 & 104 & Oklahoma & 52,228 \\
\hline 48 & Colorado & 64,388 & 105 & Alabama & 52,205 \\
\hline 49 & Columbia & 64,265 & 106 & Georgia & 51,005 \\
\hline 50 & Western Ontario & 63,872 & 107 & Wayne State & 50,031 \\
\hline 51 & Brown & 63,854 & 108 & South Carolina & 49,838 \\
\hline 52 & North Carolina & 63,750 & 109 & Southern Illinois & 49,117 \\
\hline 53 & Johns Hopkins & 63,702 & 110 & Missouri & 48,854 \\
\hline 54 & Emory & 63,653 & 111 & Ohio University & 48,170 \\
\hline 55 & Cornell & 63,600 & 112 & Louisiana State & 47,715 \\
\hline 56 & Georgetown & 63,538 & 113 & Florida State & 47,000 \\
\hline 57 & Northwestern & 62,847 & & & \\
\hline
\end{tabular}

Reprinted from ARL Annual Salary Survey 2008-2009. Salaries of directors are not included in the calculation of medians. Excludes medical and law libraries. See Tables 37 and 44 for statistics related to medical and law library salaries. Canadian salaries are expressed in US dollars. 


\section{Table 11: Median Professional Salaries in ARL University Libraries RANK ORder TABLe, FY 2009-2010}

\begin{tabular}{|c|c|c|c|c|c|}
\hline Rank & Institution & Salary & Rank & Institution & Salary \\
\hline 1 & Rutgers & 91,751 & 58 & Pennsylvania & 61,850 \\
\hline 2 & Alberta & 89,319 & 59 & Miami & 61,728 \\
\hline 3 & California, Davis & 88,488 & 60 & Iowa State & 61,664 \\
\hline 4 & Manitoba & 85,771 & 61 & Illinois, Urbana & 61,645 \\
\hline 5 & Queen`s & 83,438 & 62 & Brown & 61,549 \\
\hline 6 & California, Berkeley & 82,524 & 63 & Pittsburgh & 60,711 \\
\hline 7 & Saskatchewan & 78,850 & 64 & Syracuse & 60,648 \\
\hline 8 & Connecticut & 78,079 & 65 & Purdue & 60,513 \\
\hline 9 & York & 77,321 & 66 & Kent State & 60,301 \\
\hline 10 & Calgary & 76,749 & 67 & Duke & 60,000 \\
\hline 11 & Yale & 76,300 & 67 & Virginia & 60,000 \\
\hline 12 & California, Irvine & 75,708 & 69 & Washington & 59,934 \\
\hline 12 & California, Los Angeles & 75,708 & 70 & Arizona & 59,915 \\
\hline 12 & California, Riverside & 75,708 & 71 & Kentucky & 59,839 \\
\hline 12 & California, San Diego & 75,708 & 72 & Montreal & 59,349 \\
\hline 16 & SUNY Stony Brook & 75,457 & 73 & Indiana & 59,331 \\
\hline 17 & Toronto & 75,194 & 74 & Texas & 59,260 \\
\hline 18 & Princeton & 73,799 & 75 & SUNY Albany & 58,822 \\
\hline 19 & Delaware & 73,353 & 76 & Wisconsin & 58,654 \\
\hline 20 & Chicago & 72,329 & 77 & Virginia Tech & 58,540 \\
\hline 21 & Massachusetts & 72,263 & 78 & Washington State & 58,172 \\
\hline 22 & MIT & 70,959 & 79 & Temple & 58,140 \\
\hline 23 & Harvard & 70,720 & 80 & Georgia Tech & 58,016 \\
\hline 24 & Waterloo & 70,260 & 81 & McGill & 57,834 \\
\hline 25 & British Columbia & 70,005 & 82 & Nebraska & 57,472 \\
\hline 26 & New York University & 69,989 & 83 & Texas Tech & 57,135 \\
\hline 27 & SUNY Buffalo & 69,048 & 84 & Boston University & 56,600 \\
\hline 28 & California, Santa Barbara & 68,892 & 85 & Houston & 56,563 \\
\hline 29 & New Mexico & 68,853 & 86 & Illinois, Chicago & 56,555 \\
\hline 30 & Dartmouth & 67,933 & 87 & Florida & 56,388 \\
\hline 31 & Southern California & 67,650 & 88 & Tulane & 56,325 \\
\hline 32 & Boston College & 67,280 & 89 & Utah & 56,312 \\
\hline 33 & George Washington & 67,143 & 90 & Oregon & 55,973 \\
\hline 34 & Guelph & 66,902 & 91 & Missouri & 55,964 \\
\hline 35 & Maryland & 66,603 & 92 & Louisville & 55,884 \\
\hline 36 & Notre Dame & 66,508 & 93 & Rice & 55,833 \\
\hline 37 & Tennessee & 66,307 & 94 & Western Ontario & 55,725 \\
\hline 38 & Hawaii & 66,225 & 95 & Texas A\&M & 55,704 \\
\hline 39 & Michigan State & 65,850 & 96 & Iowa & 55,573 \\
\hline 40 & Johns Hopkins & 65,690 & 97 & Case Western Reserve & 55,227 \\
\hline 41 & Colorado State & 65,300 & 98 & Kansas & 54,806 \\
\hline 42 & Arizona State & 65,071 & 99 & Wayne State & 54,705 \\
\hline 43 & North Carolina State & 65,000 & 100 & Vanderbilt & 54,655 \\
\hline 44 & Colorado & 64,573 & 101 & Ohio State & 54,450 \\
\hline 45 & Columbia & 64,385 & 102 & Oklahoma & 53,742 \\
\hline 46 & Cornell & 64,239 & 103 & Washington U.-St. Louis & 53,740 \\
\hline 47 & Pennsylvania State & 64,224 & 104 & Auburn & 53,705 \\
\hline 48 & Brigham Young & 63,950 & 105 & Rochester & 53,000 \\
\hline 49 & Michigan & 63,717 & 106 & Oklahoma State & 52,657 \\
\hline 50 & North Carolina & 63,712 & 107 & Southern Illinois & 52,536 \\
\hline 51 & Minnesota & 63,557 & 108 & Alabama & 52,204 \\
\hline 52 & Georgetown & 63,538 & 109 & Georgia & 50,000 \\
\hline 53 & Northwestern & 63,429 & 110 & South Carolina & 49,838 \\
\hline 54 & Emory & 63,068 & 111 & Florida State & 49,805 \\
\hline 55 & McMaster & 62,508 & 112 & Ohio University & 48,787 \\
\hline 56 & Laval & 62,474 & 113 & Louisiana State & 46,462 \\
\hline 57 & Cincinnati & 61,879 & 114 & Howard & 43,010 \\
\hline
\end{tabular}

Salaries of directors are not included in the calculation of medians.

Excludes medical and law libraries. See Tables 37 and 44 for statistics related to medical and law library salaries.

Canadian salaries are expressed in US dollars. 


\section{Table 12: Average Professional Salaries in ARL University Libraries RANK ORDER TABLE, FY 2008-2009}

\begin{tabular}{|c|c|c|c|c|c|}
\hline Rank & Institution & Salary & Rank & Institution & Salary \\
\hline 1 & York & 95,755 & 58 & North Carolina & 67,177 \\
\hline 2 & Toronto & 90,829 & 59 & Purdue & 66,957 \\
\hline 3 & Manitoba & 89,270 & 60 & Cincinnati & 66,847 \\
\hline 4 & Alberta & 89,260 & 61 & Colorado & 66,826 \\
\hline 5 & Rutgers & 88,899 & 62 & Texas & 66,554 \\
\hline 6 & Queen`s & 88,107 & 63 & Pittsburgh & 66,544 \\
\hline 7 & Saskatchewan & 85,401 & 64 & Washington & 66,517 \\
\hline 8 & California, Berkeley & 82,952 & 65 & Arizona & 66,447 \\
\hline 9 & Yale & 82,622 & 66 & Northwestern & 66,328 \\
\hline 10 & British Columbia & 79,910 & 67 & Temple & 66,114 \\
\hline 11 & Connecticut & 79,855 & 68 & Pennsylvania & 65,914 \\
\hline 12 & California, Davis & 79,652 & 69 & Nebraska & 65,751 \\
\hline 13 & New York University & 78,920 & 70 & Virginia Tech & 65,516 \\
\hline 14 & McMaster & 78,776 & 71 & Syracuse & 65,441 \\
\hline 15 & New Mexico & 78,591 & 72 & Brigham Young & 63,925 \\
\hline 16 & Harvard & 78,019 & 73 & Michigan State & 63,867 \\
\hline 17 & Princeton & 77,939 & 74 & Indiana & 63,786 \\
\hline 18 & Waterloo & 77,432 & 75 & Houston & 63,512 \\
\hline 19 & Guelph & 77,319 & 76 & Iowa & 63,196 \\
\hline 20 & California, Irvine & 76,045 & 77 & Arizona State & 63,110 \\
\hline 21 & California, San Diego & 76,016 & 78 & Duke & 63,058 \\
\hline 22 & SUNY Stony Brook & 75,948 & 79 & Tulane & 62,896 \\
\hline 23 & California, Los Angeles & 75,812 & 80 & Iowa State & 61,863 \\
\hline 24 & MIT & 75,733 & 81 & Louisville & 61,851 \\
\hline 25 & Chicago & 74,480 & 82 & Rice & 61,433 \\
\hline 26 & California, Riverside & 74,166 & 83 & Illinois, Chicago & 61,206 \\
\hline 27 & Southern California & 73,788 & 84 & Utah & 61,119 \\
\hline 28 & McGill & 73,148 & 85 & Kansas & 61,056 \\
\hline 29 & Delaware & 72,792 & 86 & Georgia Tech & 60,944 \\
\hline 30 & Dartmouth & 72,539 & 87 & Kentucky & 60,909 \\
\hline 31 & Columbia & 72,352 & 88 & Texas Tech & 60,494 \\
\hline 32 & California, Santa Barbara & 71,466 & 89 & Wisconsin & 60,304 \\
\hline 33 & George Washington & 70,759 & 90 & Auburn & 60,231 \\
\hline 34 & North Carolina State & 70,667 & 91 & Washington State & 59,820 \\
\hline 35 & Massachusetts & 70,508 & 92 & Vanderbilt & 59,810 \\
\hline 36 & Tennessee & 70,344 & 93 & Washington U.-St. Louis & 59,497 \\
\hline 37 & Michigan & 70,031 & 94 & Texas A\&M & 59,250 \\
\hline 38 & SUNY Buffalo & 69,982 & 95 & Boston University & 59,236 \\
\hline 39 & Georgetown & 69,739 & 96 & Florida & 58,974 \\
\hline 40 & Boston College & 69,672 & 97 & Alabama & 58,655 \\
\hline 41 & Cornell & 69,564 & 98 & Case Western Reserve & 57,972 \\
\hline 42 & Pennsylvania State & 69,421 & 99 & Georgia & 57,628 \\
\hline 43 & Montreal & 69,342 & 100 & Ohio State & 57,439 \\
\hline 44 & Virginia & 69,246 & 101 & Kent State & 57,317 \\
\hline 45 & Miami & 69,165 & 102 & Southern Illinois & 57,003 \\
\hline 46 & Emory & 69,025 & 103 & Oregon & 56,965 \\
\hline 47 & Laval & 68,511 & 104 & Oklahoma State & 56,703 \\
\hline 48 & Colorado State & 68,355 & 105 & Rochester & 55,965 \\
\hline 49 & Notre Dame & 68,224 & 106 & Missouri & 55,264 \\
\hline 50 & Western Ontario & 68,168 & 107 & Wayne State & 54,962 \\
\hline 51 & Minnesota & 67,975 & 108 & Oklahoma & 54,228 \\
\hline 52 & Maryland & 67,924 & 109 & Ohio University & 54,210 \\
\hline 53 & Brown & 67,804 & 110 & Howard & 53,741 \\
\hline 54 & Hawaii & 67,491 & 111 & South Carolina & 53,670 \\
\hline 55 & Johns Hopkins & 67,265 & 112 & Louisiana State & 51,839 \\
\hline 56 & SUNY Albany & 67,251 & 113 & Florida State & 49,803 \\
\hline 57 & Illinois, Urbana & 67,213 & & & \\
\hline
\end{tabular}

Reprinted from ARL Annual Salary Survey 2008-2009. Salaries of directors are not included in the calculation of medians. Excludes medical and law libraries. See Tables 38 and 45 for statistics related to medical and law library salaries.

Canadian salaries are expressed in US dollars. 


\section{Table 13: Average Professional Salaries in ARL University Libraries Rank Order Table, FY 2009-2010}

\begin{tabular}{|c|c|c|c|c|c|}
\hline Rank & Institution & Salary & Rank & Institution & Salary \\
\hline 1 & Rutgers & 89,058 & 58 & Pittsburgh & 66,551 \\
\hline 2 & Alberta & 84,172 & 59 & Washington & 66,476 \\
\hline 3 & California, Berkeley & 83,853 & 60 & Arizona State & 66,091 \\
\hline 4 & York & 82,297 & 61 & Brigham Young & 66,005 \\
\hline 5 & Connecticut & 82,083 & 62 & Pennsylvania & 65,997 \\
\hline 6 & Manitoba & 81,855 & 63 & Arizona & 65,897 \\
\hline 7 & Calgary & 81,275 & 64 & Brown & 65,880 \\
\hline 8 & California, Davis & 81,135 & 65 & Syracuse & 65,847 \\
\hline 9 & Yale & 81,088 & 66 & Purdue & 65,573 \\
\hline 10 & Queen`s & 80,504 & 67 & Cincinnati & 65,445 \\
\hline 11 & SUNY Stony Brook & 79,837 & 68 & Nebraska & 65,057 \\
\hline 12 & New York University & 79,018 & 69 & Duke & 64,870 \\
\hline 13 & Princeton & 79,015 & 70 & Kent State & 63,997 \\
\hline 14 & Toronto & 78,211 & 71 & Indiana & 63,870 \\
\hline 15 & California, Los Angeles & 78,189 & 72 & McGill & 63,797 \\
\hline 16 & California, Riverside & 77,661 & 73 & Georgia Tech & 63,526 \\
\hline 17 & Harvard & 77,319 & 74 & Iowa State & 63,217 \\
\hline 18 & California, San Diego & 77,158 & 75 & Virginia Tech & 63,148 \\
\hline 19 & California, Irvine & 76,645 & 76 & Washington State & 62,755 \\
\hline 20 & Saskatchewan & 76,307 & 77 & Montreal & 62,740 \\
\hline 21 & Chicago & 76,254 & 78 & Iowa & 62,529 \\
\hline 22 & Delaware & 75,703 & 79 & Texas A\&M & 62,441 \\
\hline 23 & Southern California & 74,479 & 80 & Rice & 62,215 \\
\hline 24 & MIT & 74,430 & 81 & Illinois, Chicago & 62,208 \\
\hline 25 & George Washington & 74,353 & 82 & Laval & 61,963 \\
\hline 26 & British Columbia & 73,023 & 83 & Wisconsin & 61,856 \\
\hline 27 & North Carolina State & 73,019 & 84 & Houston & 61,669 \\
\hline 28 & Columbia & 72,939 & 85 & McMaster & 61,608 \\
\hline 29 & New Mexico & 72,938 & 86 & Utah & 61,470 \\
\hline 30 & SUNY Buffalo & 72,916 & 87 & Wayne State & 61,261 \\
\hline 31 & Dartmouth & 72,208 & 88 & Kentucky & 60,966 \\
\hline 32 & Cornell & 71,548 & 89 & SUNY Albany & 60,964 \\
\hline 33 & California, Santa Barbara & 71,378 & 90 & Kansas & 60,877 \\
\hline 34 & Waterloo & 70,230 & 91 & Texas Tech & 60,690 \\
\hline 35 & Massachusetts & 69,882 & 92 & Tulane & 60,534 \\
\hline 36 & Boston College & 69,739 & 93 & Florida & 60,450 \\
\hline 37 & Notre Dame & 69,492 & 94 & Missouri & 59,914 \\
\hline 38 & Tennessee & 69,380 & 95 & Oregon & 59,782 \\
\hline 39 & Georgetown & 69,227 & 96 & Louisville & 59,483 \\
\hline 40 & Guelph & 69,113 & 97 & Ohio State & 59,476 \\
\hline 41 & Johns Hopkins & 68,926 & 98 & Western Ontario & 59,073 \\
\hline 42 & Michigan State & 68,894 & 99 & Washington U.-St. Louis & 59,037 \\
\hline 43 & Maryland & 68,874 & 100 & Vanderbilt & 58,991 \\
\hline 44 & Emory & 68,747 & 101 & Case Western Reserve & 58,788 \\
\hline 45 & Colorado State & 68,311 & 102 & Auburn & 58,382 \\
\hline 46 & Miami & 68,278 & 103 & Alabama & 58,272 \\
\hline 47 & Virginia & 67,988 & 104 & Southern Illinois & 58,264 \\
\hline 48 & Pennsylvania State & 67,897 & 105 & Boston University & 57,615 \\
\hline 49 & Northwestern & 67,873 & 106 & Oklahoma State & 56,580 \\
\hline 50 & Michigan & 67,767 & 107 & Georgia & 56,544 \\
\hline 51 & North Carolina & 67,637 & 108 & Oklahoma & 55,853 \\
\hline 52 & Colorado & 67,523 & 109 & Rochester & 55,837 \\
\hline 53 & Texas & 66,882 & 110 & Ohio University & 55,522 \\
\hline 54 & Temple & 66,849 & 111 & Florida State & 54,858 \\
\hline 55 & Minnesota & 66,794 & 112 & South Carolina & 53,658 \\
\hline 56 & Illinois, Urbana & 66,755 & 113 & Louisiana State & 50,821 \\
\hline 57 & Hawaii & 66,721 & 114 & Howard & 43,547 \\
\hline
\end{tabular}

Salaries of directors are not included in the calculation of medians.

Excludes medical and law libraries. See Tables 38 and 45 for statistics related to medical and law library salaries. Canadian salaries are expressed in US dollars. 


\section{Table 14: Average, Median, and Beginning Professional Salaries IN ARL University LibRaRIES \\ SuMMARY OF RANKINGS, FY 2006-2007 to 2009-2010}

\begin{tabular}{|c|c|c|c|c|c|c|c|c|c|c|c|c|}
\hline \multirow{2}{*}{$\begin{array}{l}\text { Institution } \\
\\
\end{array}$} & \multicolumn{4}{|c|}{ Average Salaries } & \multicolumn{4}{|c|}{ Median Salaries } & \multicolumn{4}{|c|}{ Beginning Salaries } \\
\hline & 2007 & 2008 & 2009 & 2010 & 2007 & 2008 & 2009 & 2010 & 2007 & 2008 & 2009 & 2010 \\
\hline Alabama & 98 & 92 & 97 & 103 & 101 & 96 & 105 & 108 & 84 & 74 & 73 & 72 \\
\hline Alberta & 17 & 11 & 4 & 2 & 3 & 2 & 1 & 2 & 43 & 38 & 8 & 60 \\
\hline Arizona & 38 & 56 & 65 & 63 & 55 & 56 & 62 & 70 & 21 & 7 & 26 & 16 \\
\hline Arizona State & 69 & 59 & 77 & 60 & 66 & 41 & 66 & 42 & 23 & 34 & 60 & 61 \\
\hline Auburn & 77 & 75 & 90 & 102 & 52 & 63 & 90 & 104 & 15 & 25 & 50 & 45 \\
\hline Boston University & 104 & 106 & 40 & 105 & 97 & 100 & 91 & 84 & 112 & 62 & 70 & 57 \\
\hline Boston College & 36 & 35 & 95 & 36 & 36 & 31 & 35 & 32 & 58 & 57 & 70 & 70 \\
\hline Brigham Young & 68 & 70 & 72 & 61 & 59 & 55 & 60 & 48 & 17 & 14 & 22 & 7 \\
\hline British Columbia & 41 & 26 & 10 & 26 & 32 & 22 & 9 & 25 & 20 & 15 & 2 & 21 \\
\hline Brown & 53 & 47 & 53 & 64 & 61 & 42 & 51 & 62 & 84 & 94 & 99 & 100 \\
\hline Calgary* & N/A & $\mathrm{N} / \mathrm{A}$ & N/A & 7 & $\mathrm{~N} / \mathrm{A}$ & $\mathrm{N} / \mathrm{A}$ & N/A & 10 & N/A & $\mathrm{N} / \mathrm{A}$ & N/A & 13 \\
\hline California, Berkeley & 2 & 3 & 8 & 3 & 6 & 5 & 10 & 6 & 64 & 69 & 35 & 22 \\
\hline California, Davis & 18 & 15 & 12 & 8 & 8 & 3 & 8 & 3 & 64 & 69 & 35 & 29 \\
\hline California, Irvine & 8 & 20 & 20 & 19 & 5 & 13 & 16 & 12 & 64 & 69 & 35 & 29 \\
\hline California, Los Angeles & 5 & 10 & 23 & 15 & 7 & 12 & 19 & 12 & 64 & 69 & 35 & 29 \\
\hline California, Riverside & 29 & 28 & 26 & 16 & 22 & 24 & 18 & 12 & 26 & 41 & 27 & 22 \\
\hline California, San Diego & 11 & 14 & 21 & 18 & 10 & 13 & 16 & 12 & 44 & 69 & 35 & 29 \\
\hline California, Santa Barbara & 19 & 23 & 32 & 33 & 21 & 27 & 31 & 28 & 64 & 43 & 35 & 29 \\
\hline Case Western Reserve & 94 & 96 & 98 & 101 & 88 & 90 & 96 & 97 & 104 & 108 & 110 & 111 \\
\hline Chicago & 14 & 18 & 25 & 21 & 18 & 23 & 28 & 20 & 9 & 12 & 21 & 18 \\
\hline Cincinnati & 49 & 49 & 60 & 67 & 53 & 47 & 64 & 57 & 84 & 94 & 100 & 92 \\
\hline Colorado & 71 & 46 & 61 & 52 & 63 & 49 & 48 & 44 & 37 & 58 & 51 & 50 \\
\hline Colorado State & 55 & 68 & 48 & 45 & 41 & 53 & 45 & 41 & 45 & 29 & 42 & 39 \\
\hline Columbia & 12 & 21 & 31 & 28 & 26 & 46 & 49 & 45 & 2 & 1 & 4 & 5 \\
\hline Connecticut & 7 & 7 & 11 & 5 & 15 & 9 & 12 & 8 & 5 & 5 & 22 & 19 \\
\hline Cornell & 28 & 33 & 41 & 32 & 43 & 40 & 55 & 46 & 22 & 16 & 28 & 24 \\
\hline Dartmouth & 20 & 19 & 30 & 31 & 20 & 16 & 30 & 30 & 75 & 74 & 67 & 37 \\
\hline Delaware & 26 & 29 & 29 & 22 & 28 & 34 & 24 & 19 & 64 & 28 & 58 & 58 \\
\hline Duke & 75 & 76 & 78 & 69 & 76 & 81 & 78 & 67 & 93 & 34 & 51 & 39 \\
\hline Emory & 60 & 57 & 46 & 44 & 54 & 51 & 54 & 54 & 84 & 94 & 83 & 72 \\
\hline Florida & 93 & 100 & 96 & 93 & 93 & 98 & 92 & 87 & 27 & 44 & 73 & 72 \\
\hline Florida State & 113 & 113 & 113 & 111 & 113 & 111 & 113 & 111 & 37 & 44 & 73 & 72 \\
\hline George Washington & 40 & 54 & 33 & 25 & 62 & 62 & 44 & 33 & 27 & 44 & 28 & 24 \\
\hline Georgetown & 43 & 40 & 39 & 39 & 51 & 48 & 56 & 52 & 11 & 16 & 43 & 39 \\
\hline Georgia & 99 & 97 & 99 & 107 & 104 & 106 & 106 & 109 & 111 & 108 & 105 & 104 \\
\hline Georgia Tech & 72 & 90 & 86 & 73 & 85 & 86 & 86 & 80 & 17 & 44 & 73 & 50 \\
\hline Guelph & 56 & 69 & 19 & 40 & 56 & 66 & 23 & 34 & 80 & 90 & 1 & 6 \\
\hline Harvard & 15 & 16 & 16 & 17 & 24 & 26 & 25 & 23 & 9 & 8 & 18 & 15 \\
\hline Hawaii & 51 & 74 & 54 & 57 & 40 & 54 & 33 & 38 & 74 & 42 & 65 & 66 \\
\hline Houston & 88 & 99 & 75 & 84 & 89 & 108 & 59 & 85 & 64 & 74 & 73 & 61 \\
\hline Howard & 109 & 108 & 110 & 114 & 108 & 93 & 101 & 114 & 103 & 107 & 108 & 104 \\
\hline Illinois, Chicago & 83 & 89 & 83 & 81 & 86 & 84 & 85 & 86 & 27 & 9 & 28 & 24 \\
\hline
\end{tabular}


Table 14: Average, Median, and Beginning Professional Salaries IN ARL UnIVERSITY LIBRARIES

SuMMARY OF RANKINGS, FY 2006-2007 to 2009-2010

\begin{tabular}{|c|c|c|c|c|c|c|c|c|c|c|c|c|}
\hline Institution & \multicolumn{4}{|c|}{ Average Salaries } & \multicolumn{4}{|c|}{ Median Salaries } & \multicolumn{4}{|c|}{ Beginning Salaries } \\
\hline FY & 2007 & 2008 & 2009 & 2010 & 2007 & 2008 & 2009 & 2010 & 2007 & 2008 & 2009 & 2010 \\
\hline Illinois, Urbana & 54 & 62 & 57 & 56 & 71 & 68 & 61 & 61 & 23 & 26 & 41 & 34 \\
\hline Indiana & 65 & 60 & 74 & 71 & 64 & 60 & 70 & 73 & 92 & 74 & 90 & 90 \\
\hline Iowa & 67 & 65 & 76 & 78 & 83 & 79 & 83 & 96 & 45 & 74 & 85 & 83 \\
\hline Iowa State & 76 & 82 & 80 & 74 & 72 & 75 & 74 & 60 & 37 & 39 & 60 & 61 \\
\hline Johns Hopkins & 44 & 44 & 55 & 41 & 46 & 57 & 53 & 40 & 7 & 4 & 9 & 10 \\
\hline Kansas & 86 & 86 & 85 & 90 & 78 & 82 & 87 & 98 & 45 & 74 & 16 & 7 \\
\hline Kent State & 96 & 101 & 101 & 70 & 94 & 92 & 99 & 66 & 1 & 31 & 59 & 2 \\
\hline Kentucky & 91 & 85 & 87 & 88 & 81 & 71 & 76 & 71 & 81 & 91 & 85 & 83 \\
\hline Laval & 97 & 84 & 47 & 82 & 73 & 39 & 29 & 56 & 94 & 68 & 24 & 68 \\
\hline Louisiana State & 112 & 112 & 112 & 113 & 112 & 113 & 112 & 113 & 99 & 94 & 105 & 104 \\
\hline Louisville & 48 & 51 & 81 & 96 & 27 & 43 & 82 & 92 & 99 & 100 & 107 & 109 \\
\hline McGill & 39 & 43 & 28 & 72 & 17 & 32 & 34 & 81 & 59 & 65 & 13 & 65 \\
\hline McMaster & 64 & 37 & 14 & 85 & 38 & 20 & 11 & 55 & 96 & 89 & 49 & 91 \\
\hline Manitoba & 9 & 5 & 3 & 6 & 4 & 4 & 2 & 4 & 71 & 55 & 33 & 81 \\
\hline Maryland & 61 & 55 & 52 & 43 & 50 & 44 & 39 & 35 & 45 & 44 & 73 & 92 \\
\hline Massachusetts & 25 & 27 & 35 & 35 & 11 & 19 & 21 & 21 & 60 & 63 & 72 & 71 \\
\hline MIT & 24 & 25 & 24 & 24 & 29 & 29 & 25 & 22 & 11 & 5 & 6 & 4 \\
\hline Miami & 42 & 52 & 45 & 46 & 65 & 58 & 46 & 59 & 36 & 16 & 43 & 39 \\
\hline Michigan & 35 & 41 & 37 & 50 & 45 & 36 & 41 & 49 & 45 & 58 & 73 & 72 \\
\hline Michigan State & 59 & 71 & 73 & 42 & 47 & 76 & 71 & 39 & 6 & 9 & 28 & 24 \\
\hline Minnesota & 37 & 42 & 51 & 55 & 39 & 35 & 43 & 51 & 75 & 74 & 91 & 72 \\
\hline Missouri & 105 & 95 & 106 & 94 & 102 & 104 & 110 & 91 & 108 & 91 & 91 & 92 \\
\hline Montreal & 87 & 78 & 43 & 77 & 80 & 69 & 38 & 72 & 107 & 104 & 82 & 110 \\
\hline Nebraska & 78 & 73 & 69 & 68 & 87 & 87 & 80 & 82 & 41 & 16 & 10 & 11 \\
\hline New Mexico & 13 & 9 & 15 & 29 & 9 & 8 & 15 & 29 & 45 & 74 & 91 & 92 \\
\hline New York & 10 & 4 & 13 & 12 & 31 & 21 & 27 & 26 & 2 & 2 & 3 & 3 \\
\hline North Carolina & 47 & 34 & 58 & 51 & 44 & 37 & 52 & 50 & 27 & 44 & 51 & 50 \\
\hline North Carolina State & 27 & 30 & 34 & 27 & 49 & 45 & 47 & 43 & 8 & 9 & 16 & 11 \\
\hline Northwestern & 50 & 50 & 66 & 49 & 42 & 50 & 57 & 53 & 84 & 86 & 73 & 61 \\
\hline Notre Dame & 32 & 38 & 49 & 37 & 30 & 28 & 40 & 36 & 83 & 74 & 91 & 82 \\
\hline Ohio University & 111 & 111 & 100 & 110 & 111 & 112 & 111 & 112 & 104 & 105 & 83 & 83 \\
\hline Ohio State & 85 & 98 & 109 & 97 & 79 & 95 & 103 & 101 & 41 & 54 & 60 & 34 \\
\hline Oklahoma & 110 & 107 & 108 & 108 & 110 & 103 & 104 & 102 & 45 & 74 & 73 & 72 \\
\hline Oklahoma State & 101 & 104 & 104 & 106 & 100 & 105 & 100 & 106 & 99 & 105 & 108 & 104 \\
\hline Oregon & 107 & 103 & 103 & 95 & 103 & 102 & 98 & 90 & 104 & 108 & 91 & 92 \\
\hline Pennsylvania & 52 & 66 & 68 & 62 & 48 & 64 & 65 & 58 & 27 & 39 & 67 & 59 \\
\hline Pennsylvania State & 34 & 39 & 42 & 48 & 37 & 38 & 42 & 47 & 45 & 56 & 69 & 69 \\
\hline Pittsburgh & 62 & 61 & 63 & 58 & 69 & 65 & 73 & 63 & 108 & 111 & 111 & 112 \\
\hline Princeton & 16 & 13 & 17 & 13 & 23 & 18 & 22 & 18 & 45 & 16 & 10 & 1 \\
\hline Purdue & 63 & 48 & 59 & 66 & 82 & 72 & 66 & 65 & 23 & 29 & 43 & 24 \\
\hline Queen's & 23 & 17 & 6 & 10 & 14 & 11 & 5 & 5 & 73 & 64 & 13 & 48 \\
\hline
\end{tabular}


Table 14: Average, Median, and Beginning Professional Salaries

IN ARL UNIVERSITY LIBRARIES

SuMMARY OF RANKINGS, FY 2006-2007 to 2009-2010

\begin{tabular}{|c|c|c|c|c|c|c|c|c|c|c|c|c|}
\hline \multirow{2}{*}{$\begin{array}{l}\text { Institution } \\
\\
\end{array}$} & \multicolumn{4}{|c|}{ Average Salaries } & \multicolumn{4}{|c|}{ Median Salaries } & \multicolumn{4}{|c|}{ Beginning Salaries } \\
\hline & 2007 & 2008 & 2009 & 2010 & 2007 & 2008 & 2009 & 2010 & 2007 & 2008 & 2009 & 2010 \\
\hline Rice & 82 & 72 & 82 & 80 & 92 & 83 & 89 & 93 & 98 & 85 & 103 & 108 \\
\hline Rochester & 102 & 102 & 105 & 109 & 99 & 101 & 102 & 105 & 95 & 103 & 104 & 103 \\
\hline Rutgers & 1 & 1 & 5 & 1 & 1 & 1 & 3 & 1 & 16 & 13 & 25 & 14 \\
\hline Saskatchewan & 31 & 12 & 7 & 20 & 25 & 10 & 7 & 7 & 102 & 53 & 12 & 38 \\
\hline South Carolina & 108 & 110 & 111 & 112 & 106 & 109 & 108 & 110 & 108 & 111 & 111 & 112 \\
\hline Southern California & 21 & 22 & 27 & 23 & 33 & 33 & 36 & 31 & 11 & 16 & 20 & 17 \\
\hline Southern Illinois & 100 & 105 & 102 & 104 & 107 & 107 & 109 & 107 & 61 & 58 & 60 & 50 \\
\hline SUNY Albany & 80 & 77 & 56 & 89 & 68 & 77 & 75 & 75 & 72 & 94 & 101 & 101 \\
\hline SUNY Buffalo & 81 & 58 & 38 & 30 & 84 & 52 & 37 & 27 & 11 & 16 & 43 & 39 \\
\hline SUNY Stony Brook & 22 & 24 & 22 & 11 & 19 & 25 & 20 & 16 & 27 & 34 & 51 & 72 \\
\hline Syracuse & 58 & 53 & 71 & 65 & 74 & 67 & 63 & 64 & 113 & N/A & 113 & N/A \\
\hline Temple & 46 & 45 & 67 & 54 & 57 & 78 & 88 & 79 & 75 & 86 & 64 & 49 \\
\hline Tennessee & 45 & 32 & 36 & 38 & 34 & 30 & 32 & 37 & 45 & 44 & 51 & 50 \\
\hline Texas & 57 & 64 & 62 & 53 & 67 & 73 & 77 & 74 & 17 & 16 & 43 & 34 \\
\hline Texas A\&M & 92 & 79 & 94 & 79 & 96 & 85 & 97 & 95 & 27 & 27 & 28 & 20 \\
\hline Texas Tech & 106 & 81 & 88 & 91 & 105 & 94 & 93 & 83 & 75 & 16 & 43 & 39 \\
\hline Toronto & 4 & 8 & 2 & 14 & 2 & 6 & 4 & 17 & 34 & 33 & 5 & 46 \\
\hline Tulane & 73 & 87 & 79 & 92 & 60 & 80 & 71 & 88 & 84 & 100 & 91 & 92 \\
\hline Utah & 90 & 80 & 84 & 86 & 91 & 88 & 81 & 89 & 45 & 44 & 51 & 50 \\
\hline Vanderbilt & 89 & 94 & 92 & 100 & 95 & 97 & 94 & 100 & 75 & 86 & 91 & 88 \\
\hline Virginia & 33 & 36 & 44 & 47 & 35 & 58 & 68 & 67 & 35 & 34 & 51 & 50 \\
\hline Virginia Tech & 74 & 67 & 70 & 75 & 70 & 61 & 58 & 77 & 84 & 91 & 85 & 92 \\
\hline Washington & 70 & 63 & 64 & 59 & 75 & 70 & 69 & 69 & 45 & 58 & 66 & 67 \\
\hline Washington State & 79 & 91 & 91 & 76 & 77 & 89 & 84 & 78 & 81 & 94 & 101 & 102 \\
\hline Washington U.-St. Louis & 84 & 93 & 93 & 99 & 90 & 99 & 95 & 103 & 84 & 100 & 91 & 92 \\
\hline Waterloo & 30 & 31 & 18 & 34 & 16 & 17 & 13 & 24 & 63 & 52 & 15 & 47 \\
\hline Wayne State & 103 & 109 & 107 & 87 & 109 & 110 & 107 & 99 & 45 & 67 & 89 & 88 \\
\hline Western Ontario & 95 & 88 & 50 & 98 & 98 & 91 & 50 & 94 & 40 & 65 & 34 & 86 \\
\hline Wisconsin & 66 & 83 & 89 & 83 & 58 & 74 & 79 & 76 & 62 & 84 & 88 & 87 \\
\hline Yale & 3 & 6 & 9 & 9 & 13 & 15 & 14 & 11 & 4 & 3 & 7 & 9 \\
\hline York & 6 & 2 & 1 & 4 & 12 & 7 & 6 & 9 & 97 & 32 & 19 & 80 \\
\hline
\end{tabular}

Excludes medical and law libraries.

* Calgary became a member in FY 2010. 
Page Intentionally Left Blank.

ARL University Libraries · 39 


\section{Table 15: Distribution of Professional Staff in ARL University Libraries by SAlary and Position, FY 2009-2010}

\begin{tabular}{|c|c|c|c|c|c|c|c|c|c|c|c|c|c|c|c|c|c|c|c|c|c|c|c|c|c|c|c|c|c|c|c|c|}
\hline & 离 & & 8 & & & 80 & & 8 & $8=$ & $\because \approx$ & 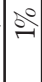 & ลें & ले & $=$ & & ले & ते & ले: & 2 & 8 & +2 & in 3 & $\circ: 0$ & $\therefore$ & 50 & in & की & ले & & & & छे \\
\hline & 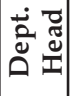 & & 8 & & 8 & 80 & 8 & 50? & तै & Sீ) & 8 & ूे। & 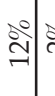 & हैं & 3 & ले & की & मी & 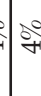 & 80 & ले शे & ले ले & กิ & ถิำ & $\stackrel{2}{-1}$ & ถి & $=20$ & 의 & 805 & 5 & & 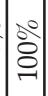 \\
\hline & 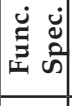 & & & & & & & & $\Rightarrow$ & ते & ले & भे & 60 & $=8$ & $m^{\circ}$ & 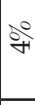 & & लेखे & in & in & 50 & to & 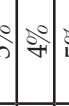 & in 5 & in & 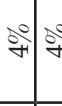 & +े ले & की & 80 & & & : \\
\hline 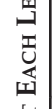 & $\begin{array}{l}\dot{0} \\
\dot{\Xi} \\
\omega \\
\omega\end{array}$ & & & & 8 & 8 & 8 & 8 & $\because 8$ & ㄴำ & N & \$ & in & $=8$ & 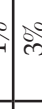 & ले & ले & ले & 9 & 8 & 50 & in & & (8) & 3: & iे & +2 & ले & 50 & 95 & & $8^{\circ}$ \\
\hline $\begin{array}{l}\vec{z} \\
\vec{u} \\
\underline{u} \\
\vec{t} \\
z\end{array}$ & 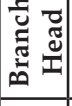 & & 8 & & $8^{\circ}$ & & & $\therefore$ & & 20 & $\infty$ & $\infty$ & : & तै। & i़ & in & की & in & ले & की & इ্ ले & ลิำ & & ㅇำ & 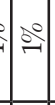 & 500 & $=80$ & 8ㅇ & 8ㅇ & & & छे \\
\hline E. & 安. & & & $\neg$ & $\AA^{\circ}$ & 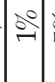 & & $=$ & वे: & $\forall$ & $\delta^{\circ}$ & 30 & ஸे & $=0$ & 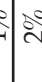 & ले & 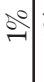 & की: & 10 & $\therefore$ & $\therefore$ & 50 & $\therefore \approx$ & $\therefore$ & & & & & & & & 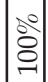 \\
\hline & 宽. & & 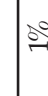 & ले & in & 80 & $\stackrel{8}{=}$ & 0 & $\stackrel{0}{0}$ & तै & in & ถેे & ले & 5 & 5 & 8 & $0^{\circ}$ & & & 80 & 의 & :5) & & & & & & & & & & ఫి \\
\hline & $\dot{\vec{\theta}}$ & +2 & 8 & ) & $\approx$ & अं & 8ః & की & & & & & & & & & & & & & & & & & & & & & & & & ¿े \\
\hline & 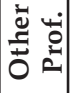 & & - & & & -1 & -1 & & 0 & $\vec{A}$ & $\widehat{\omega}$ & $\infty$ & హ) & הి & $\stackrel{4}{H}$ & 1 & की & $5)$ & $\stackrel{4}{\circ}$ & $\overrightarrow{9}$ & $\Xi$ & 늘 & $=$ & 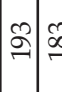 &  & If & 式 $\infty$ & 지 & 위 & & & $\left|\begin{array}{c}1 \\
0 \\
0 \\
\end{array}\right|$ \\
\hline & 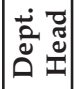 & & - & & N & & + & $\theta$ & तે & 83 & $\infty$ & $\underset{\exists}{ }$ & : & i) & 5 & f & $\left|\begin{array}{l}\infty \\
1 \\
1\end{array}\right|$ & 全 & 8 & 하 & 교 & 위 ले & $\hat{~}$ & $\vec{\lambda}$ & 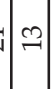 & & $\exists \sigma$ & $\wedge$ & & 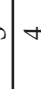 & & 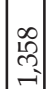 \\
\hline & 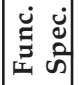 & & & & & & & & 요 & $\vec{\nabla} \mid \approx$ & 18 & 위 & $8=$ & $\pm ?$ & $\infty$ & $\mathscr{q}$ & & ભ & in & $\infty$ & 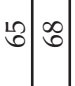 & ட & 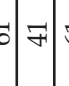 & 78 & $\mathrm{~S}$ & F & 可贫 & $\exists$ & $\rightarrow \infty$ & & & 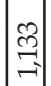 \\
\hline 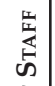 & 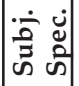 & & & & - & & $m$ & & $\Rightarrow i$ & હે & Fे & $\infty$ & ș & $\Delta \pi$ & 4 in & 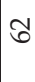 & $\mathrm{s}$ & $\widehat{6}$ & $\stackrel{\circ}{\wedge}$ & 잉 & $\infty 2 \mathbb{్}$ & $\Xi$ & & $\stackrel{\infty}{\exists}$ & 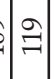 & $\exists \infty$ & sin & 남 & & & & $\mid \begin{array}{c}2 \\
\vec{i} \\
\mathbf{i}\end{array}$ \\
\hline 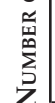 & 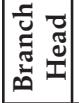 & & - & & N & & $m$ & & $=$ & ले & 와 & ले & 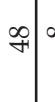 & $\infty$. & $1 \div$ & $\underset{\sim}{2}$ & तㅅ & त) & $\triangle$ & ते & $\approx A$ & $\therefore ᄋ$ & $=8$ & 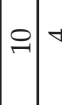 & $\infty$ & in & \begin{tabular}{l|l}
0 & -1
\end{tabular} & $N \mid$ & - & & & $\mid \begin{array}{c}\hat{\infty} \\
\stackrel{+}{f}\end{array}$ \\
\hline & 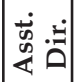 & & & & $\infty$ & - & $\infty$ & $\infty$ & त) & $\stackrel{\sim}{\sharp}$ & $=$ & $\Rightarrow$ & $\infty$ & $N$ & 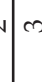 & in & $N$ & 0. & - & $\infty$ & - & & $\| \sim$ & 4 & & & & & & & & 기 \\
\hline & 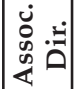 & & & & 요 & $\stackrel{\infty}{\sim}$ & ले| & & & $\Sigma \underset{\Sigma}{\infty}$ & $\therefore$ & $\wedge$ & $\infty$. & & - & -1 & $\mathrm{~N}$ & & & - & $N$ & $-1-$ & & & & & & & & & & $\mid$ \\
\hline & $\ddot{a}$ & & 0 & & ( & t & & $\forall$ & & & & & & & & & & & & & & & & & & & & & & & & \pm \\
\hline & 童 & 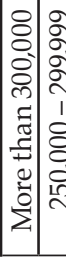 & 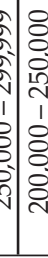 & 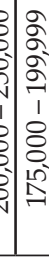 & 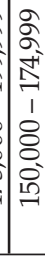 & $\left|\begin{array}{l}2 \\
2 \\
2 \\
+ \\
1 \\
8 \\
8 \\
0 \\
= \\
=\end{array}\right|$ & 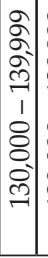 & 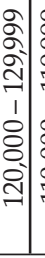 & 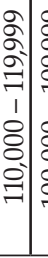 & 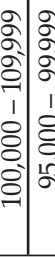 & $\begin{array}{l}\sigma \\
\alpha \\
0 \\
1 \\
0 \\
8 \\
\delta \\
\delta\end{array}$ & $\begin{array}{l}2 \\
2 \\
2 \\
\infty \\
1 \\
8 \\
8 \\
15 \\
\infty\end{array}$ & 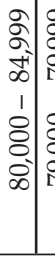 & 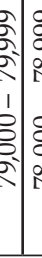 & 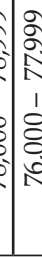 & \begin{tabular}{l}
2 \\
$\alpha$ \\
1 \\
1 \\
1 \\
8 \\
8 \\
\multirow{2}{*}{}
\end{tabular} & 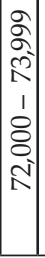 & 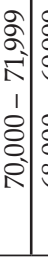 & $\mid \begin{array}{l}2 \\
8 \\
1 \\
1 \\
8 \\
8 \\
8 \\
0\end{array}$ & 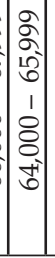 & 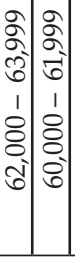 & 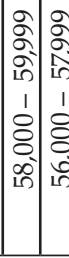 & 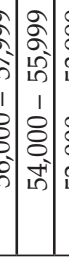 & 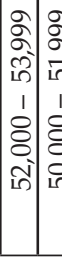 & 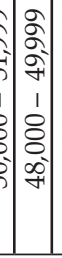 & 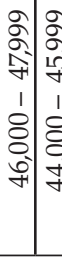 & 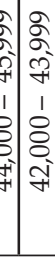 & 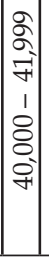 & 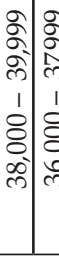 & 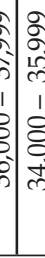 & 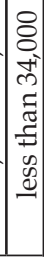 & 式 \\
\hline
\end{tabular}

Excludes medical and law libraries.

† A " 0 " percentage indicates less than one-half of one percent. 


\section{Table 16: Distribution of Professional Staff in ARL University Libraries by Salary, SeX, and Position, FY 2009-2010}

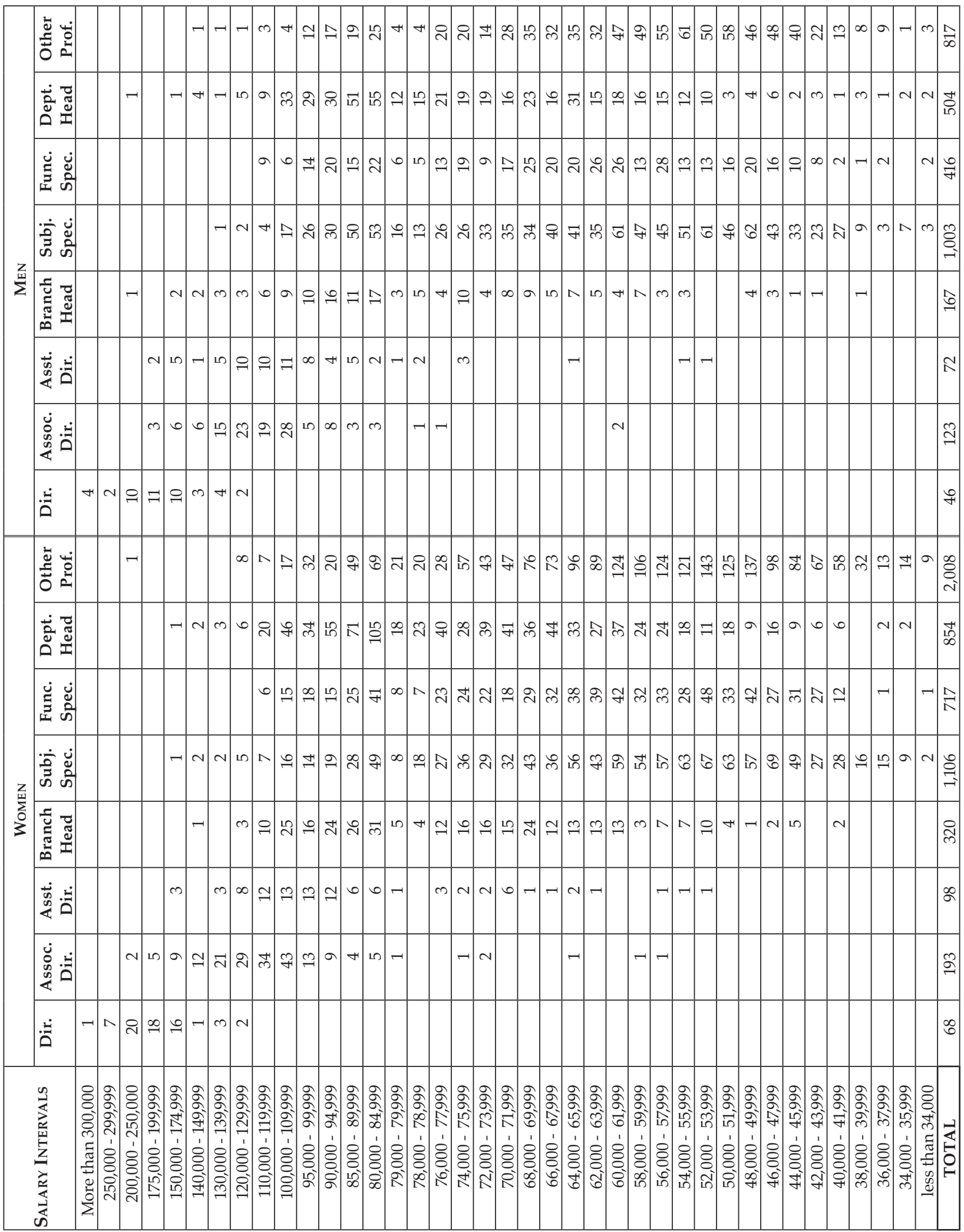

Excludes medical and law libraries. 


\section{Table 17: Number and Average Salaries of ARL University Librarians by Position ANd Sex, FY 2009-2010}

\begin{tabular}{|c|c|c|c|c|c|c|}
\hline \multirow[b]{2}{*}{ Position } & \multicolumn{2}{|c|}{ WOMEN } & \multicolumn{2}{|c|}{ Men } & \multicolumn{2}{|c|}{ TotaL } \\
\hline & Salary & No. & Salary & No. & Salary & No. \\
\hline Director & $\$ 197,433$ & 68 & $\$ 196,188$ & 46 & $\$ 196,930$ & 114 \\
\hline Associate Director & 117,708 & 193 & 116,845 & 123 & 117,372 & 316 \\
\hline Assistant Director & 97,331 & 98 & 109,862 & 72 & 102,639 & 170 \\
\hline Head, Branch & 78,079 & 320 & 82,727 & 167 & 79,673 & 487 \\
\hline Functional Specialist & 62,070 & 1,106 & 64,299 & 1,003 & 63,130 & 2,109 \\
\hline Subject Specialist & 64,332 & 717 & 67,459 & 416 & 65,480 & 1,133 \\
\hline \multicolumn{7}{|l|}{ Dept. Head: } \\
\hline Acquisitions & 74,986 & 79 & 70,942 & 33 & 73,794 & 112 \\
\hline Reference & 77,320 & 78 & 80,958 & 30 & 78,331 & 108 \\
\hline Cataloging & 74,317 & 109 & 74,251 & 40 & 74,299 & 149 \\
\hline Serials & 75,123 & 21 & 69,354 & 9 & 73,392 & 30 \\
\hline Documents/Maps & 68,333 & 36 & 70,020 & 23 & 68,990 & 59 \\
\hline Circulation & 72,934 & 56 & 64,168 & 27 & 70,082 & 83 \\
\hline Rare Books/Manuscripts & 80,587 & 40 & 83,992 & 50 & 82,479 & 90 \\
\hline Computer Systems & 92,577 & 26 & 88,277 & 47 & 89,809 & 73 \\
\hline Other & 76,631 & 409 & 78,638 & 245 & 77,382 & 654 \\
\hline \multicolumn{7}{|l|}{ Reference: } \\
\hline Over 14 years experience & 68,883 & 403 & 66,505 & 173 & 68,169 & 576 \\
\hline 10 to 14 years experience & 60,293 & 157 & 60,397 & 55 & 60,320 & 212 \\
\hline 5 to 9 years experience & 55,217 & 215 & 55,692 & 91 & 55,358 & 306 \\
\hline Under 5 years experience & 48,558 & 195 & 48,739 & 59 & 48,600 & 254 \\
\hline \multicolumn{7}{|l|}{ Cataloging: } \\
\hline Over 14 years experience & 65,088 & 269 & 66,199 & 132 & 65,454 & 401 \\
\hline 10 to 14 years experience & 57,127 & 77 & 61,197 & 29 & 58,240 & 106 \\
\hline 5 to 9 years experience & 54,661 & 93 & 55,275 & 48 & 54,870 & 141 \\
\hline Under 5 years experience & 49,693 & 81 & 48,978 & 31 & 49,496 & 112 \\
\hline \multicolumn{7}{|l|}{ Other: } \\
\hline Over 14 years experience & 68,142 & 221 & 68,260 & 78 & 68,173 & 299 \\
\hline 10 to 14 years experience & 61,221 & 88 & 59,435 & 35 & 60,713 & 123 \\
\hline 5 to 9 years experience & 55,932 & 95 & 58,224 & 36 & 56,562 & 131 \\
\hline Under 5 years experience & 49,709 & 114 & 51,033 & 50 & 50,112 & 164 \\
\hline All Positions & $\$ 69,277$ & 5,364 & $\$ 71,953$ & 3,148 & $\$ 70,266$ & 8,512 \\
\hline
\end{tabular}

Canadian salaries are expressed in US dollars. See Table 32 for salaries of Canadian librarians expressed in Canadian dollars. Excludes medical and law libraries. See Tables 39 and 46 for salaries in medical and law libraries. 


\section{Table 18: Number and Average Years of Experience of ARL University Librarians by Position AND Sex, FY 2009-2010}

\begin{tabular}{|c|c|c|c|c|c|c|}
\hline \multirow[b]{2}{*}{ Position } & \multicolumn{2}{|c|}{ WOMEN } & \multicolumn{2}{|c|}{ Men } & \multicolumn{2}{|c|}{ TOTAL } \\
\hline & Years & No. & Years & No. & Years & No. \\
\hline Director & 33.3 & 68 & 32.5 & 46 & 33.0 & 114 \\
\hline Associate Director & 26.7 & 193 & 23.8 & 123 & 25.6 & 316 \\
\hline Assistant Director & 24.5 & 98 & 24.2 & 72 & 24.4 & 170 \\
\hline Head, Branch & 21.6 & 320 & 22.9 & 167 & 22.0 & 487 \\
\hline Functional Specialist & 13.9 & 1,106 & 13.4 & 1,003 & 13.6 & 2,109 \\
\hline Subject Specialist & 16.1 & 717 & 17.0 & 416 & 16.4 & 1,133 \\
\hline \multicolumn{7}{|l|}{ Dept. Head: } \\
\hline Acquisitions & 21.5 & 79 & 16.8 & 33 & 20.2 & 112 \\
\hline Reference & 20.0 & 78 & 21.1 & 30 & 20.3 & 108 \\
\hline Cataloging & 22.2 & 109 & 21.0 & 40 & 21.8 & 149 \\
\hline Serials & 21.0 & 21 & 20.2 & 9 & 20.8 & 30 \\
\hline Documents/Maps & 19.8 & 36 & 21.4 & 23 & 20.4 & 59 \\
\hline Circulation & 20.0 & 56 & 16.1 & 27 & 18.8 & 83 \\
\hline Rare Books/Manuscripts & 21.5 & 40 & 23.9 & 50 & 22.8 & 90 \\
\hline Computer Systems & 20.3 & 26 & 19.3 & 47 & 19.7 & 73 \\
\hline Other & 20.6 & 409 & 19.3 & 245 & 20.1 & 654 \\
\hline Public Services & 13.3 & 221 & 12.3 & 89 & 13.0 & 310 \\
\hline Technical Services & 15.6 & 139 & 14.9 & 49 & 15.4 & 188 \\
\hline Administrative Services & 15.2 & 158 & 13.4 & 61 & 14.7 & 219 \\
\hline Reference & 14.6 & 970 & 15.1 & 378 & 14.8 & 1,348 \\
\hline Cataloger & 17.0 & 520 & 17.9 & 240 & 17.3 & 760 \\
\hline All Positions & 17.1 & 5,364 & 16.9 & 3,148 & 17.0 & 8,512 \\
\hline
\end{tabular}

Includes Canadian libraries. See Table 33 for comparable figures in Canadian libraries only.

Excludes medical and law libraries. See Tables 40 and 47 for comparable figures in medical and law libraries. 
Table 19: Number and Average Salaries of ARL University Librarians

by Years of EXPerience AND SeX, FY 2009-2010

\begin{tabular}{|c|c|c|c|c|c|c|c|}
\hline \multirow[b]{2}{*}{ Experience } & \multicolumn{2}{|c|}{ WOMEN } & \multicolumn{2}{|c|}{ Men } & \multicolumn{2}{|c|}{ Total } & \multirow{2}{*}{$\begin{array}{r}\% \text { OF } \\
\text { TotaL }\end{array}$} \\
\hline & Salary & No. & Salary & No. & Salary & No. & \\
\hline $0-3$ years & $\$ 48,872$ & 623 & $\$ 50,893$ & 339 & $\$ 49,584$ & 962 & $11 \%$ \\
\hline $4-7$ years & 54,555 & 701 & 56,165 & 409 & 55,148 & 1,110 & $13 \%$ \\
\hline $8-11$ years & 60,758 & 750 & 63,540 & 489 & 61,856 & 1,239 & $15 \%$ \\
\hline $12-15$ years & 66,656 & 583 & 68,728 & 369 & 67,459 & 952 & $11 \%$ \\
\hline 16 - 19 years & 70,357 & 534 & 74,583 & 319 & 71,938 & 853 & $10 \%$ \\
\hline $20-23$ years & 74,484 & 543 & 77,753 & 312 & 75,677 & 855 & $10 \%$ \\
\hline $24-27$ years & 79,661 & 481 & 82,459 & 243 & 80,600 & 724 & $9 \%$ \\
\hline 28 - 31 years & 82,859 & 415 & 87,795 & 277 & 84,835 & 692 & $8 \%$ \\
\hline 32 - 35 years & 90,399 & 368 & 96,132 & 217 & 92,526 & 585 & $7 \%$ \\
\hline over 35 years & 94,251 & 366 & 95,301 & 174 & 94,589 & 540 & $6 \%$ \\
\hline All Positions & $\$ 69,277$ & 5,364 & $\$ 71,953$ & 3,148 & $\$ 70,266$ & 8,512 & $100 \%$ \\
\hline
\end{tabular}

Canadian salaries are expressed in US dollars. See Table 34 for salaries in Canadian dollars.

Excludes medical and law libraries. See Tables 41 and 48 for salaries in medical and law libraries. 


\section{Table 20: Average Salaries of ARL University Librarians by Position and YeARs of Experience, FY 2009-2010}

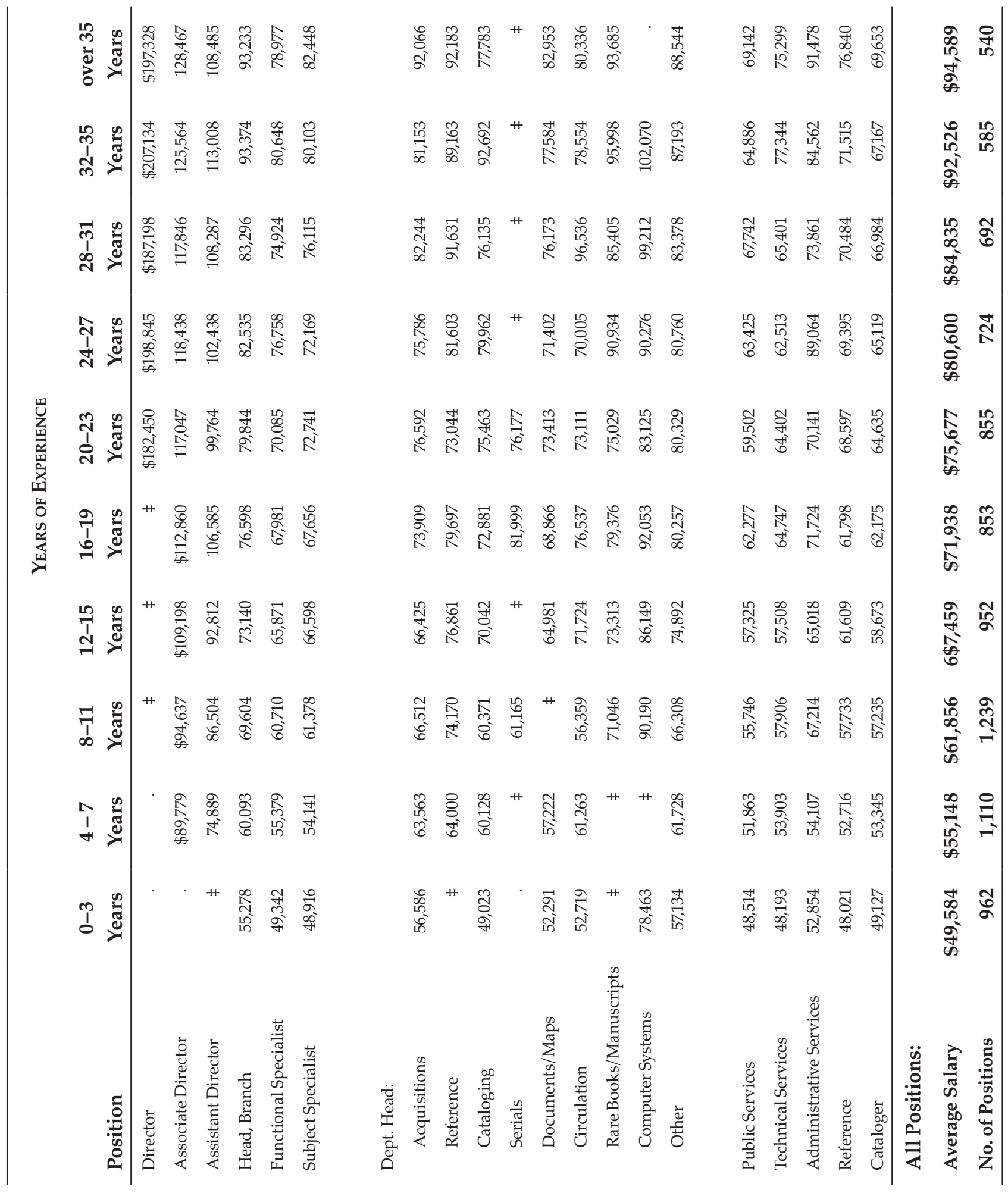

Years of experience reflect total professional experience. Canadian salaries are expressed in US dollars. Excludes medical and law libraries. ₹ Salary data are not published when fewer than four individuals are involved.

. No positions reported in this category. 


\section{Table 21: Number and Average Salaries of ARL University Librarians by Position AND Type of INSTITUTion, FY 2009-2010}

\begin{tabular}{|c|c|c|c|c|c|c|c|c|}
\hline \multirow[b]{2}{*}{ Position } & \multicolumn{2}{|c|}{ CANADian (15) } & \multicolumn{2}{|c|}{ Private (31) } & \multicolumn{2}{|c|}{ Public (68) } & \multicolumn{2}{|c|}{ TотаL (114) } \\
\hline & Salary & No. & Salary & No. & Salary & No. & Salary & No. \\
\hline Director & $\$ 151,500$ & 15 & $\$ 233,154$ & 31 & $\$ 190,438$ & 68 & $\$ 196,930$ & 114 \\
\hline Associate Director & 109,191 & 40 & 127,387 & 96 & 113,848 & 180 & 117,372 & 316 \\
\hline Assistant Director & 91,609 & 19 & 108,281 & 67 & 100,633 & 84 & 102,639 & 170 \\
\hline Head, Branch & 86,859 & 60 & 83,934 & 132 & 76,304 & 295 & 79,673 & 487 \\
\hline Functional Specialist & 65,785 & 180 & 66,631 & 781 & 60,332 & 1,148 & 63,130 & 2,109 \\
\hline Subject Specialist & 65,527 & 123 & 66,563 & 396 & 64,772 & 614 & 65,480 & 1,133 \\
\hline \multicolumn{9}{|l|}{ Dept. Head: } \\
\hline Acquisitions & 83,456 & 15 & 74,788 & 28 & 71,291 & 69 & 73,794 & 112 \\
\hline Reference & 72,514 & 15 & 81,465 & 41 & 77,538 & 52 & 78,331 & 108 \\
\hline Cataloging & 80,090 & 11 & 77,153 & 56 & 71,573 & 82 & 74,299 & 149 \\
\hline Serials & 78,717 & 4 & 73,339 & 11 & 72,011 & 15 & 73,392 & 30 \\
\hline Documents/Maps & 83,961 & 8 & 65,169 & 14 & 67,199 & 37 & 68,990 & 59 \\
\hline Circulation & 69,888 & 14 & 73,250 & 18 & 69,018 & 51 & 70,082 & 83 \\
\hline Rare Books/Manuscripts & 83,843 & 8 & 80,276 & 30 & 83,539 & 52 & 82,479 & 90 \\
\hline Computer Systems & 82,756 & 7 & 88,599 & 23 & 91,604 & 43 & 89,809 & 73 \\
\hline Other & 80,848 & 67 & 79,580 & 201 & 75,637 & 386 & 77,382 & 654 \\
\hline \multicolumn{9}{|l|}{ Reference: } \\
\hline Over 14 years experience & 80,653 & 83 & 66,413 & 150 & 65,916 & 343 & 68,169 & 576 \\
\hline 10 to 14 years experience & 70,692 & 29 & 59,948 & 61 & 58,041 & 122 & 60,320 & 212 \\
\hline 5 to 9 years experience & 61,424 & 71 & 57,477 & 67 & 51,949 & 168 & 55,358 & 306 \\
\hline Under 5 years experience & 54,104 & 61 & 49,291 & 48 & 46,056 & 145 & 48,600 & 254 \\
\hline \multicolumn{9}{|l|}{ Cataloging: } \\
\hline Over 14 years experience & 73,160 & 37 & 66,179 & 165 & 63,420 & 199 & 65,454 & 401 \\
\hline 10 to 14 years experience & 57,874 & 10 & 60,295 & 47 & 56,344 & 49 & 58,240 & 106 \\
\hline 5 to 9 years experience & 63,124 & 9 & 58,906 & 64 & 49,979 & 68 & 54,870 & 141 \\
\hline Under 5 years experience & 53,444 & 10 & 52,198 & 48 & 46,362 & 54 & 49,496 & 112 \\
\hline \multicolumn{9}{|l|}{ Other: } \\
\hline Over 14 years experience & 77,347 & 17 & 65,366 & 115 & 69,172 & 167 & 68,173 & 299 \\
\hline 10 to 14 years experience & 63,580 & 10 & 65,394 & 40 & 57,755 & 73 & 60,713 & 123 \\
\hline 5 to 9 years experience & 58,862 & 12 & 61,027 & 51 & 52,808 & 68 & 56,562 & 131 \\
\hline Under 5 years experience & 56,512 & 12 & 52,935 & 53 & 47,825 & 99 & 50,112 & 164 \\
\hline All Positions & $\$ 73,363$ & 947 & $\$ 72,455$ & 2,834 & $\$ 68,335$ & 4,731 & $\$ 70,266$ & 8,512 \\
\hline
\end{tabular}

Canadian salaries are expressed in US dollars. Tables 31-34 show Canadian salaries in Canadian dollars. Excludes medical and law libraries.

( ) Indicates the number of ARL libraries in each category.

₹ Salary data are not published when fewer than four individuals are involved. 
Table 22: Years of Experience of ARL University Librarians

by Position ANd Type of Institution, FY 2009-2010

\begin{tabular}{|c|c|c|c|c|c|c|c|c|}
\hline \multirow[b]{2}{*}{ Position } & \multicolumn{2}{|c|}{ CANADIAN (15) } & \multicolumn{2}{|c|}{ Private (31) } & \multicolumn{2}{|c|}{ Public (68) } & \multicolumn{2}{|c|}{ TOTAL (114) } \\
\hline & Years & No. & Years & No. & Years & No. & Years & No. \\
\hline Director & 32.0 & 15 & 32.9 & 31 & 33.3 & 68 & 33.0 & 114 \\
\hline Associate Director & 23.3 & 40 & 26.2 & 96 & 25.8 & 180 & 25.6 & 316 \\
\hline Assistant Director & 24.2 & 19 & 25.2 & 67 & 23.8 & 84 & 24.4 & 170 \\
\hline Head, Branch & 22.0 & 60 & 22.3 & 132 & 22.0 & 295 & 22.0 & 487 \\
\hline Functional Specialist & 14.4 & 180 & 14.0 & 781 & 13.2 & 1,148 & 13.6 & 2,109 \\
\hline Subject Specialist & 14.7 & 123 & 17.2 & 396 & 16.3 & 614 & 16.4 & 1,133 \\
\hline \multicolumn{9}{|l|}{ Dept. Head: } \\
\hline Acquisitions & 21.1 & 15 & 20.9 & 28 & 19.7 & 69 & 20.2 & 112 \\
\hline Reference & 18.9 & 15 & 21.2 & 41 & 20.1 & 52 & 20.3 & 108 \\
\hline Cataloging & 24.0 & 11 & 21.8 & 56 & 21.6 & 82 & 21.8 & 149 \\
\hline Serials & 24.0 & 4 & 24.3 & 11 & 17.4 & 15 & 20.8 & 30 \\
\hline Documents/Maps & 25.3 & 8 & 17.2 & 14 & 20.5 & 37 & 20.4 & 59 \\
\hline Circulation & 18.7 & 14 & 16.5 & 18 & 19.6 & 51 & 18.8 & 83 \\
\hline Rare Books/Manuscripts & 23.9 & 8 & 21.1 & 30 & 23.6 & 52 & 22.8 & 90 \\
\hline Computer Systems & 20.7 & 7 & 18.8 & 23 & 20.0 & 43 & 19.7 & 73 \\
\hline Other & 18.3 & 67 & 20.4 & 201 & 20.2 & 386 & 20.1 & 654 \\
\hline \multicolumn{9}{|l|}{ Reference: } \\
\hline Over 14 years experience & 25.6 & 83 & 25.7 & 150 & 25.1 & 343 & 25.3 & 576 \\
\hline 10 to 14 years experience & 12.0 & 29 & 11.9 & 61 & 11.9 & 122 & 11.9 & 212 \\
\hline 5 to 9 years experience & 6.9 & 71 & 7.1 & 67 & 7.1 & 168 & 7.0 & 306 \\
\hline Under 5 years experience & 2.3 & 61 & 2.6 & 48 & 2.6 & 145 & 2.5 & 254 \\
\hline \multicolumn{9}{|l|}{ Cataloging: } \\
\hline Over 14 years experience & 29.2 & 37 & 26.0 & 165 & 26.4 & 199 & 26.5 & 401 \\
\hline 10 to 14 years experience & 12.4 & 10 & 11.6 & 47 & 12.3 & 49 & 12.0 & 106 \\
\hline 5 to 9 years experience & 6.9 & 9 & 7.0 & 64 & 6.6 & 68 & 6.8 & 141 \\
\hline Under 5 years experience & 2.0 & 10 & 2.4 & 48 & 2.6 & 54 & 2.5 & 112 \\
\hline \multicolumn{9}{|l|}{ Other: } \\
\hline Over 14 years experience & 25.3 & 17 & 23.4 & 115 & 25.6 & 167 & 24.7 & 299 \\
\hline 10 to 14 years experience & 12.1 & 10 & 11.7 & 40 & 11.8 & 73 & 11.8 & 123 \\
\hline 5 to 9 years experience & 7.5 & 12 & 6.9 & 51 & 7.1 & 68 & 7.1 & 131 \\
\hline Under 5 years experience & 1.8 & 12 & 2.5 & 53 & 2.4 & 99 & 2.4 & 164 \\
\hline All Positions & 16.5 & 947 & 17.2 & 2,834 & 17.0 & 4,731 & 17.0 & 8,512 \\
\hline
\end{tabular}

Excludes medical and law libraries.

( ) Indicates the number of ARL libraries in each category. 


\section{Table 23: Number and Average Salaries of ARL University Librarians by Position and Size of Professional Staff, FY 2009-2010}

\begin{tabular}{|c|c|c|c|c|c|c|c|c|}
\hline \multirow[b]{2}{*}{ Position } & \multicolumn{2}{|c|}{$\begin{array}{c}\text { STAFF OVER } 110 \\
(\mathbf{1 7})^{+}\end{array}$} & \multicolumn{2}{|c|}{$\begin{array}{c}\text { STAFF 75-110 } \\
\text { (18) }\end{array}$} & \multicolumn{2}{|c|}{$\begin{array}{c}\text { StAFF 50-74 } \\
(47)\end{array}$} & \multicolumn{2}{|c|}{$\begin{array}{c}\text { STAFF } 26-49 \\
(32)^{\ddagger}\end{array}$} \\
\hline & Salary & No. & Salary & No. & Salary & No. & Salary & No. \\
\hline Director & $\$ 231,501$ & 17 & $\$ 210,533$ & 18 & $\$ 189,054$ & 47 & $\$ 182,482$ & 32 \\
\hline Associate Director & 134,748 & 70 & 119,139 & 77 & 109,188 & 108 & 109,691 & 61 \\
\hline Assistant Director & 115,943 & 46 & 88,907 & 34 & 101,340 & 67 & 100,112 & 23 \\
\hline Head, Branch & 84,603 & 167 & 82,727 & 78 & 76,016 & 163 & 73,782 & 79 \\
\hline Functional Specialist & 65,859 & 785 & 66,668 & 408 & 59,080 & 651 & 59,548 & 265 \\
\hline Subject Specialist & 69,550 & 380 & 68,675 & 209 & 62,060 & 451 & 58,252 & 93 \\
\hline \multicolumn{9}{|l|}{ Dept. Head: } \\
\hline Acquisitions & 75,996 & 23 & 84,002 & 16 & 70,314 & 48 & 71,917 & 25 \\
\hline Reference & 85,296 & 31 & 74,170 & 8 & 74,368 & 41 & 77,612 & 28 \\
\hline Cataloging & 80,354 & 50 & 74,170 & 19 & 68,369 & 52 & 73,610 & 28 \\
\hline Serials & 73,900 & 12 & 71,703 & 5 & 78,001 & 6 & 69,779 & 7 \\
\hline Documents/Maps & 70,362 & 15 & 71,369 & 9 & 69,968 & 20 & 64,888 & 15 \\
\hline Circulation & 76,734 & 22 & 71,560 & 16 & 66,193 & 31 & 66,553 & 14 \\
\hline Rare Books/Manuscripts & 95,586 & 13 & 87,079 & 17 & 77,219 & 34 & 79,794 & 26 \\
\hline Computer Systems & 104,411 & 13 & 96,813 & 10 & 83,427 & 36 & 87,657 & 14 \\
\hline Other & 81,749 & 187 & 78,049 & 125 & 74,009 & 256 & 76,960 & 86 \\
\hline \multicolumn{9}{|l|}{ Reference: } \\
\hline Over 14 years experience & 68,964 & 132 & 69,578 & 113 & 67,048 & 180 & 67,756 & 151 \\
\hline 10 to 14 years experience & 61,504 & 56 & 61,308 & 36 & 60,023 & 71 & 58,673 & 49 \\
\hline 5 to 9 years experience & 59,880 & 73 & 56,085 & 70 & 52,506 & 90 & 53,655 & 73 \\
\hline Under 5 years experience & 51,246 & 44 & 50,882 & 48 & 46,687 & 104 & 48,136 & 58 \\
\hline \multicolumn{9}{|l|}{ Cataloging: } \\
\hline Over 14 years experience & 70,998 & 151 & 62,239 & 80 & 60,649 & 122 & 65,583 & 48 \\
\hline 10 to 14 years experience & 64,400 & 41 & 54,950 & 16 & 54,489 & 36 & 53,251 & 13 \\
\hline 5 to 9 years experience & 59,274 & 62 & 52,141 & 27 & 51,655 & 33 & 49,961 & 19 \\
\hline Under 5 years experience & 54,235 & 45 & 47,118 & 17 & 46,203 & 40 & 45,378 & 10 \\
\hline \multicolumn{9}{|l|}{ Other: } \\
\hline Over 14 years experience & 71,771 & 93 & 73,263 & 66 & 65,847 & 91 & 58,806 & 49 \\
\hline 10 to 14 years experience & 64,406 & 40 & 62,684 & 37 & 59,384 & 27 & 50,988 & 19 \\
\hline 5 to 9 years experience & 61,041 & 49 & 55,350 & 32 & 54,324 & 35 & 49,739 & 15 \\
\hline Under 5 years experience & 51,805 & 60 & 50,375 & 39 & 49,903 & 50 & 43,356 & 15 \\
\hline
\end{tabular}

Canadian salaries are expressed in US dollars. For average Canadian salaries (expressed in US dollars) refer to Table 21; Tables 31-34 show Canadian salaries in Canadian dollars.

Excludes medical and law libraries.

( ) Indicates the number of ARL libraries in each category.

† In 1995-1996 and earlier, the first column of this table reported staff over 124; in 1996-1998 over 120; in 1998-1999 over 115; and since 1999-2000, over 110.

\# No ARL library has fewer than 26 professional staff members. 
Table 24: Years of Experience of ARL University Librarians

by Position and Size of Professional Staff, FY 2009-2010

\begin{tabular}{|c|c|c|c|c|c|c|c|c|}
\hline \multirow[b]{2}{*}{ Position } & \multicolumn{2}{|c|}{$\begin{array}{c}\text { StAFF OVER } 110 \\
(\mathbf{1 7})^{\dagger}\end{array}$} & \multicolumn{2}{|c|}{$\begin{array}{c}\text { StAFF } 75-110 \\
(18)\end{array}$} & \multicolumn{2}{|c|}{$\begin{array}{c}\text { STAFF } 50-74 \\
(47)\end{array}$} & \multicolumn{2}{|c|}{$\begin{array}{c}\text { STAFF 26-49 } \\
(32)^{\ddagger}\end{array}$} \\
\hline & Years & No. & Years & No. & Years & No. & Years & No \\
\hline Director & 34.7 & 17 & 32.1 & 18 & 32.4 & 47 & 33.4 & 32 \\
\hline Associate Director & 26.4 & 70 & 25.9 & 77 & 24.6 & 108 & 26.1 & 61 \\
\hline Assistant Director & 25.7 & 46 & 21.9 & 34 & 24.3 & 67 & 25.4 & 23 \\
\hline Head, Branch & 22.4 & 167 & 21.6 & 78 & 21.9 & 163 & 22.0 & 79 \\
\hline Functional Specialist & 13.3 & 785 & 14.1 & 408 & 13.6 & 651 & 14.0 & 265 \\
\hline Subject Specialist & 16.1 & 380 & 17.3 & 209 & 16.8 & 451 & 14.1 & 93 \\
\hline \multicolumn{9}{|l|}{ Dept. Head: } \\
\hline Acquisitions & 20.8 & 23 & 24.3 & 16 & 19.2 & 48 & 18.7 & 25 \\
\hline Reference & 21.8 & 31 & 18.5 & 8 & 20.2 & 41 & 19.4 & 28 \\
\hline Cataloging & 22.7 & 50 & 22.3 & 19 & 21.9 & 52 & 20.0 & 28 \\
\hline Serials & 23.8 & 12 & 17.0 & 5 & 21.7 & 6 & 17.6 & 7 \\
\hline Documents/Maps & 21.3 & 15 & 19.0 & 9 & 20.8 & 20 & 19.9 & 15 \\
\hline Circulation & 20.3 & 22 & 16.1 & 16 & 18.5 & 31 & 19.9 & 14 \\
\hline Rare Books/Manuscripts & 24.8 & 13 & 22.2 & 17 & 22.4 & 34 & 22.8 & 26 \\
\hline Computer Systems & 20.4 & 13 & 22.3 & 10 & 17.8 & 36 & 21.9 & 14 \\
\hline Other & 22.1 & 187 & 18.6 & 125 & 18.9 & 256 & 21.5 & 86 \\
\hline \multicolumn{9}{|l|}{ Reference: } \\
\hline Over 14 years experience & 26.4 & 132 & 25.5 & 113 & 25.0 & 180 & 24.6 & 151 \\
\hline 10 to 14 years experience & 12.1 & 56 & 11.8 & 36 & 11.9 & 71 & 11.9 & 49 \\
\hline 5 to 9 years experience & 7.2 & 73 & 6.8 & 70 & 7.0 & 90 & 7.2 & 73 \\
\hline Under 5 years experience & 2.4 & 44 & 2.8 & 48 & 2.3 & 104 & 2.8 & 58 \\
\hline \multicolumn{9}{|l|}{ Cataloging: } \\
\hline Over 14 years experience & 26.5 & 151 & 25.8 & 80 & 27.0 & 122 & 26.2 & 48 \\
\hline 10 to 14 years experience & 11.8 & 41 & 12.2 & 16 & 12.1 & 36 & 12.2 & 13 \\
\hline 5 to 9 years experience & 6.9 & 62 & 6.8 & 27 & 6.8 & 33 & 6.9 & 19 \\
\hline Under 5 years experience & 2.6 & 45 & 2.1 & 17 & 2.6 & 40 & 2.2 & 10 \\
\hline \multicolumn{9}{|l|}{ Other: } \\
\hline Over 14 years experience & 25.5 & 93 & 24.9 & 66 & 25.0 & 91 & 22.7 & 49 \\
\hline 10 to 14 years experience & 12.0 & 40 & 11.8 & 37 & 11.8 & 27 & 11.3 & 19 \\
\hline 5 to 9 years experience & 7.0 & 49 & 7.3 & 32 & 6.9 & 35 & 7.3 & 15 \\
\hline Under 5 years experience & 2.5 & 60 & 2.3 & 39 & 2.4 & 50 & 2.1 & 15 \\
\hline All Positions & 16.9 & 2,677 & 16.9 & 1,630 & 17.1 & 2,890 & 17.5 & 1,315 \\
\hline
\end{tabular}

Excludes medical and law libraries.

() Indicates the number of ARL libraries in each category.

† In 1995-1996 and earlier, the first column of this table reported staff over 124; in 1996-1998 over 120; in 1998-1999 over 115; and since 1999-2000, over 110.

‡ No ARL library has fewer than 26 professional staff members. 


\section{Table 25: Average Salaries of ARL University Librarians by Position and Geographic Region, FY 2009-2010}

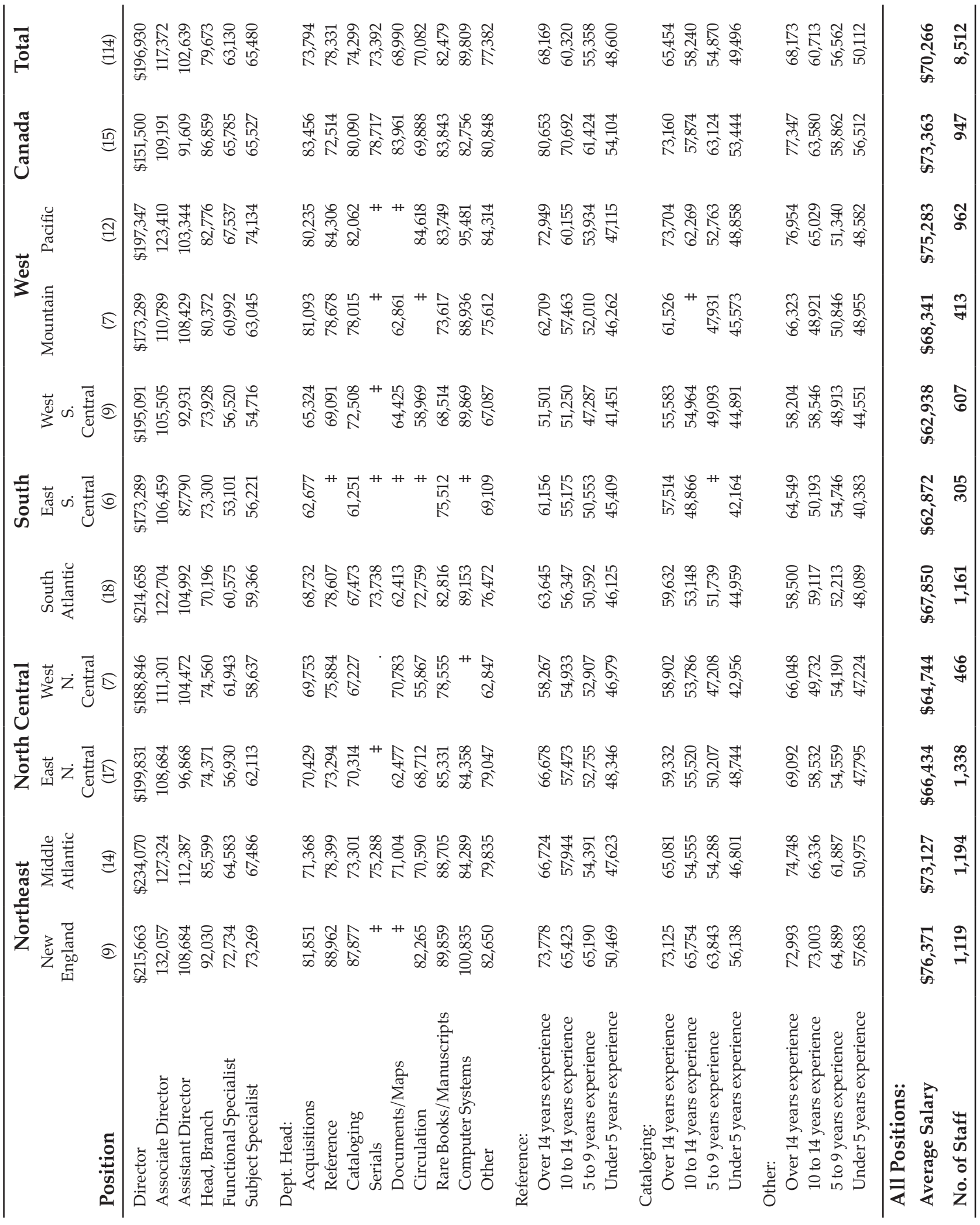

Canadian salaries are expressed in US dollars. Excludes medical and law libraries.

( ) Indicates number of ARL libraries included.

₹ Salary data are not published when fewer than four individuals are involved. 


\section{ARL University Libraries by GeOgraphic Region}

\begin{tabular}{|c|c|c|}
\hline Region & $\begin{array}{c}\text { No. of } \\
\text { Libraries }\end{array}$ & ARL University Libraries Included \\
\hline
\end{tabular}

Northeast

1. New England

2. Middle Atlantic

14

North Central

3. East North Central

4. West North Central

South

5. South Atlantic

6. East South Central

7. West South Central

9

7

17

Case Western Reserve, Chicago, Cincinnati, Illinois-Chicago, Illinois-Urbana, Indiana, Kent State, Michigan, Michigan State, Notre Dame, Northwestern, Ohio University, Ohio State, Purdue, Southern Illinois, Wayne State, Wisconsin

Iowa, Iowa State, Kansas, Minnesota, Missouri, Nebraska, Washington U.-St. Louis

18 Delaware, Duke, Emory, Florida, Florida State, Georgia, Georgia Tech., Georgetown, George Washington, Howard, Johns Hopkins, Maryland, Miami, North Carolina, North Carolina State, South Carolina, Virginia, Virginia Tech

6

Alabama, Auburn, Kentucky, Louisville, Tennessee, Vanderbilt

Houston, Louisiana State, Oklahoma, Oklahoma State, Rice, Texas, Texas A\&M, Texas Tech, Tulane

\section{West}

8. Mountain

Arizona, Arizona State, Brigham Young, Colorado, Colorado State, New Mexico, Utah

9. Pacific

12

University of California: Berkeley, Davis, Irvine, Los Angeles, Riverside, San Diego, Santa Barbara; Hawaii; Oregon; Southern California; Washington; Washington State

\section{Canada} 15
Alberta, British Columbia, Calgary, Guelph, Laval, McGill, McMaster, Manitoba, Montreal, Queen's, Saskatchewan, Toronto, Waterloo, Western Ontario, York
Conn., Mass., Me., N.H., R.I., Vt.

N.J., N.Y., Pa.

Ill., Ind., Mich., Ohio, Wis.

Iowa, Kan., Minn., Mo., Neb., N. Dak., S. Dak.

Del., DC, Fla., Ga., Md., N.C., S.C., Va., W. Va.

Ala., Ky., Miss., Tenn.

Ark., La., Okla., Tex.

Ariz., Colo., Idaho, Mont., Nev., N. Mex., Utah, Wyo.

Alaska, Calif., Hawaii, Ore., Wash.

Alta., B.C., Man., N. Br., Newf., N.S., Ont., P.E.I., Que., Sask.

Regions are based on the classification used by the US Bureau of the Census in tabulations of the Current Population Survey. 



\section{US ARL UNIVERSITY LIBRARIES}

TABles 26-30 


\section{Table 26: Average Salaries of US ARL University Librarians by Position AND YeArs of EXPerience, FY 2009-2010}

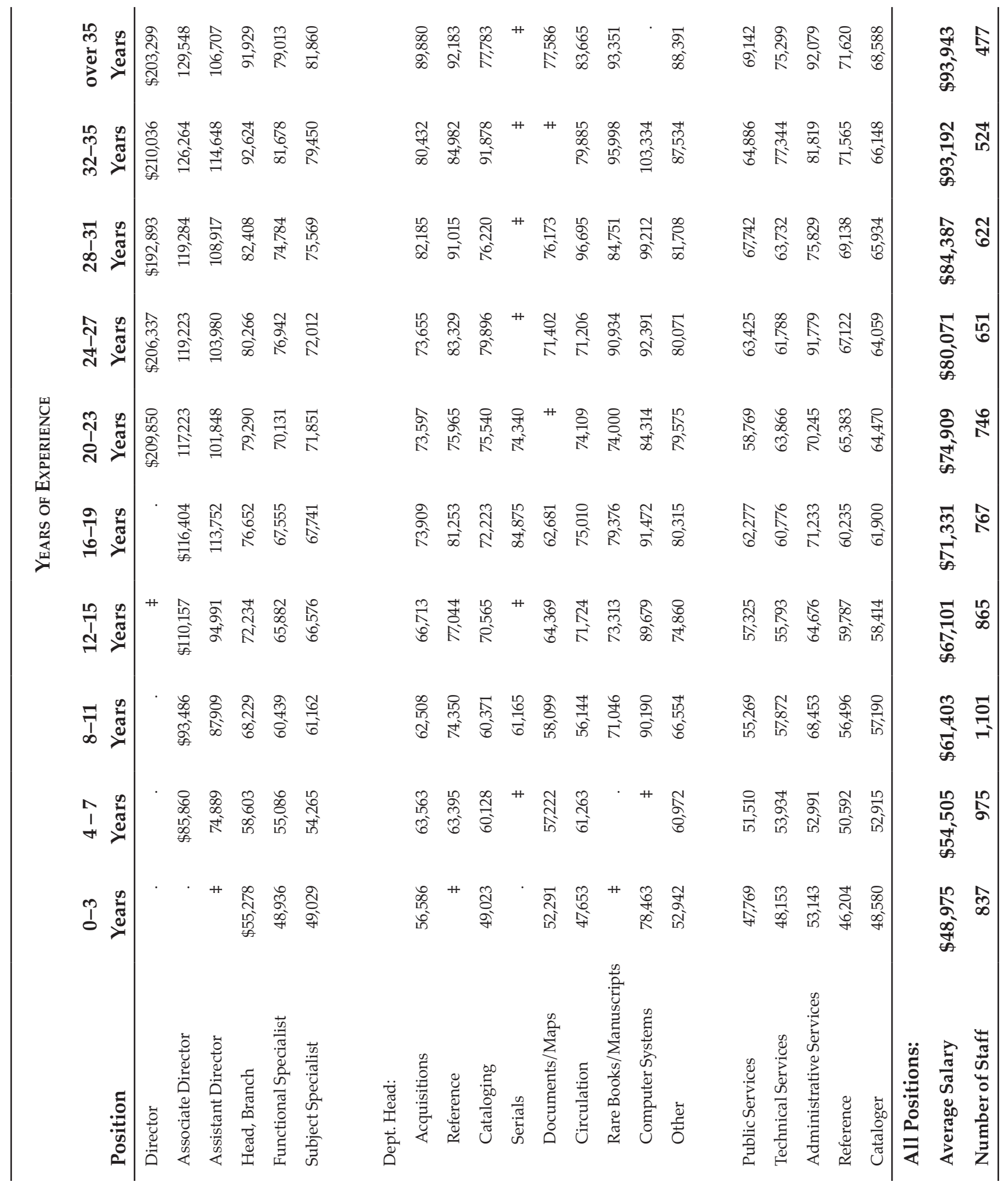

Excludes Canadian libraries.

Excludes medical and law libraries.

₹ Salary data are not published when fewer than four individuals are involved.

. No positions reported in this category. 

Table 27: Number and Average Salaries of Minority US ARL University Librarians
By Position and Sex, FY 2009-2010

\begin{tabular}{|c|c|c|c|c|c|c|}
\hline \multirow[b]{2}{*}{ Position } & \multicolumn{2}{|c|}{ WOMEN } & \multicolumn{2}{|c|}{ Men } & \multicolumn{2}{|c|}{ Total } \\
\hline & Salary & No. & Salary & No. & Salary & No. \\
\hline Director & $\neq$ & 4 & $\neq$ & 3 & $\$ 174,493$ & 7 \\
\hline Associate Director & $\neq$ & 17 & $\neq$ & 3 & 117,635 & 20 \\
\hline Assistant Director & $\neq$ & 3 & $\neq$ & 6 & 106,970 & 9 \\
\hline Head, Branch & 70,496 & 32 & 88,641 & 14 & 76,019 & 46 \\
\hline Functional Specialist & 59,836 & 142 & 63,696 & 123 & 61,628 & 265 \\
\hline Subject Specialist & 63,406 & 140 & 65,048 & 60 & 63,898 & 200 \\
\hline \multicolumn{7}{|l|}{ Dept. Head: } \\
\hline Acquisitions & $\neq$ & 7 & $\neq$ & 2 & 75,881 & 9 \\
\hline Reference & 75,228 & 5 & . & & 75,228 & 5 \\
\hline Cataloging & $\neq$ & 13 & $\neq$ & 1 & 72,247 & 14 \\
\hline Serials & $\neq$ & 2 & . & & $\neq$ & 2 \\
\hline Documents/Maps & $\neq$ & 2 & $\ddagger$ & 1 & $\neq$ & 3 \\
\hline Circulation & $\neq$ & 5 & $\neq$ & 3 & 73,467 & 8 \\
\hline Rare Books/Manuscripts & $\neq$ & 5 & $\neq$ & 2 & 75,803 & 7 \\
\hline Computer Systems & $\neq$ & 3 & $\neq$ & 8 & 82,953 & 11 \\
\hline Other & 75,136 & 42 & 78,975 & 17 & 76,242 & 59 \\
\hline \multicolumn{7}{|l|}{ Reference: } \\
\hline Over 14 years experience & 69,371 & 44 & 67,086 & 17 & 68,734 & 61 \\
\hline 10 to 14 years experience & 61,337 & 13 & 59,038 & 7 & 60,532 & 20 \\
\hline 5 to 9 years experience & 53,947 & 29 & 57,244 & 7 & 54,588 & 36 \\
\hline Under 5 years experience & 46,929 & 31 & 47,963 & 8 & 47,141 & 39 \\
\hline \multicolumn{7}{|l|}{ Cataloging: } \\
\hline Over 14 years experience & 62,158 & 40 & 71,254 & 12 & 64,257 & 52 \\
\hline 10 to 14 years experience & 59,078 & 14 & 61,525 & 6 & 59,812 & 20 \\
\hline 5 to 9 years experience & 52,110 & 22 & 55,600 & 10 & 53,201 & 32 \\
\hline Under 5 years experience & $\neq$ & 21 & $\neq$ & 3 & 50,312 & 24 \\
\hline \multicolumn{7}{|l|}{ Other: } \\
\hline Over 14 years experience & 55,135 & 31 & 61,786 & 12 & 56,991 & 43 \\
\hline 10 to 14 years experience & 53,124 & 11 & 45,823 & 4 & 51,177 & 15 \\
\hline 5 to 9 years experience & 51,928 & 19 & 71,544 & 5 & 56,015 & 24 \\
\hline Under 5 years experience & 45,929 & 22 & 50,295 & 4 & 46,601 & 26 \\
\hline All Positions & $\$ 63,452$ & 719 & $\$ 67,786$ & 338 & $\$ 64,838$ & 1,057 \\
\hline
\end{tabular}

Excludes Canadian libraries.

Excludes medical and law libraries.

\# Salary data are not published when fewer than four individuals are involved in either category.

. No positions reported in this category. 


\section{Table 28: Number and Average Years of Experience of MinORITY US ARL University LibraRians \\ by Position AND Sex, FY 2009-2010}

\begin{tabular}{|c|c|c|c|c|c|c|}
\hline \multirow[b]{2}{*}{ Position } & \multicolumn{2}{|c|}{ WoMen } & \multicolumn{2}{|c|}{ MeN } & \multicolumn{2}{|c|}{ TOTAL } \\
\hline & Years & No. & Years & No. & Years & No. \\
\hline Director & 29.0 & 4 & 31.3 & 3 & 30.0 & 7 \\
\hline Associate Director & 27.5 & 17 & 24.0 & 3 & 27.0 & 20 \\
\hline Assistant Director & 18.0 & 3 & 16.2 & 6 & 16.8 & 9 \\
\hline Head, Branch & 21.2 & 32 & 18.6 & 14 & 20.4 & 46 \\
\hline Functional Specialist & 13.2 & 142 & 11.9 & 123 & 12.6 & 265 \\
\hline Subject Specialist & 14.5 & 140 & 12.9 & 60 & 14.0 & 200 \\
\hline \multicolumn{7}{|l|}{ Dept. Head: } \\
\hline Acquisitions & 16.1 & 7 & 5.5 & 2 & 13.8 & 9 \\
\hline Reference & 15.6 & 5 & . & & 15.6 & 5 \\
\hline Cataloging & 18.8 & 13 & 9.0 & 1 & 18.1 & 14 \\
\hline Serials & 17.0 & 2 & . & & 17.0 & 2 \\
\hline Documents/Maps & 6.5 & 2 & 12.0 & 1 & 8.3 & 3 \\
\hline Circulation & 15.6 & 5 & 26.3 & 3 & 19.6 & 8 \\
\hline Rare Books/Manuscripts & 15.6 & 5 & 18.5 & 2 & 16.4 & 7 \\
\hline Computer Systems & 18.0 & 3 & 13.1 & 8 & 14.5 & 11 \\
\hline Other & 20.6 & 42 & 20.0 & 17 & 20.4 & 59 \\
\hline Public Services & 9.8 & 42 & 12.5 & 15 & 10.5 & 57 \\
\hline Technical Services & 15.6 & 26 & 15.0 & 1 & 15.6 & 27 \\
\hline Administrative Services & 11.3 & 15 & 12.0 & 9 & 11.6 & 24 \\
\hline Reference & 13.1 & 117 & 14.2 & 39 & 13.4 & 156 \\
\hline Cataloger & 14.0 & 97 & 16.2 & 31 & 14.6 & 128 \\
\hline All Positions & 14.8 & 719 & 14.0 & 338 & 14.5 & 1,057 \\
\hline
\end{tabular}

Includes Canadian libraries. See Table 33 for comparable figures in Canadian libraries only.

Excludes medical and law libraries. See Tables 40 and 47 for comparable figures in medical and law libraries.

. No positions reported in this category. 
Table 29: Number and Average Salaries

OF US ARL UNIVERSITY LIBRARIANS

by YeARS of EXPERIENCE AND SEX, FY 2009-2010

\begin{tabular}{lccccccc}
\hline & \multicolumn{2}{c}{ WoMEN } & \multicolumn{2}{c}{ MEN } & \multicolumn{2}{c}{ Total } & \multicolumn{2}{c}{$\%$ OF } \\
Experience & Salary & No. & Salary & No. & Salary & No. & TotAL \\
\hline $0-3$ years & $\$ 48,193$ & 532 & $\$ 50,339$ & 305 & $\$ 48,975$ & 837 & $11 \%$ \\
$4-7$ years & 53,685 & 606 & 55,851 & 369 & 54,505 & 975 & $13 \%$ \\
$8-11$ years & 60,117 & 658 & 63,315 & 443 & 61,403 & 1,101 & $15 \%$ \\
$12-15$ years & 66,030 & 526 & 68,763 & 339 & 67,101 & 865 & $11 \%$ \\
$16-19$ years & 69,772 & 477 & 73,896 & 290 & 71,331 & 767 & $10 \%$ \\
$20-23$ years & 73,665 & 476 & 77,102 & 270 & 74,909 & 746 & $10 \%$ \\
$24-27$ years & 78,812 & 433 & 82,572 & 218 & 80,071 & 651 & $9 \%$ \\
$28-31$ years & 82,158 & 373 & 87,725 & 249 & 84,387 & 622 & $8 \%$ \\
$32-35$ years & 91,022 & 325 & 96,735 & 199 & 93,192 & 524 & $7 \%$ \\
over 35 years & 93,602 & 319 & 94,632 & 158 & 93,943 & 477 & $6 \%$ \\
\hline All Positions & $\$ 68,787$ & 4,725 & $\$ 71,695$ & $\mathbf{2 , 8 4 0}$ & $\$ 69,879$ & 7,565 & $\mathbf{1 0 0} \%$ \\
\hline
\end{tabular}

Excludes Canadian libraries.

Excludes medical and law libraries.

Table 30: Number and Average Salaries

of Minority US ARL University LibraRIans

by Years of Experience and SeX, FY 2009-2010

\begin{tabular}{lccccccc}
\hline & \multicolumn{2}{c}{ WoMEN } & \multicolumn{2}{c}{ MEN } & \multicolumn{2}{c}{ TotaL } & \multicolumn{2}{c}{$\%$ OF } \\
Experience & Salary & No. & Salary & No. & Salary & No. & TotAL \\
\hline 0-3 years & $\$ 49,086$ & 108 & $\$ 52,603$ & 50 & $\$ 50,199$ & 158 & $15 \%$ \\
$4-7$ years & 52,179 & 117 & 57,931 & 48 & 53,852 & 165 & $16 \%$ \\
$8-11$ years & 59,063 & 97 & 66,489 & 74 & 62,277 & 171 & $16 \%$ \\
$12-15$ years & 64,348 & 92 & 67,103 & 51 & 65,330 & 143 & $14 \%$ \\
$16-19$ years & 67,679 & 82 & 75,925 & 31 & 69,941 & 113 & $11 \%$ \\
$20-23$ years & 74,658 & 73 & 72,769 & 24 & 74,190 & 97 & $9 \%$ \\
$24-27$ years & 64,148 & 52 & 77,502 & 15 & 67,138 & 67 & $6 \%$ \\
$28-31$ years & 72,729 & 29 & 79,092 & 18 & 75,166 & 47 & $4 \%$ \\
$32-35$ years & 92,311 & 38 & 82,115 & 10 & 90,186 & 48 & $5 \%$ \\
over 35 years & 84,323 & 31 & 97,108 & 17 & 88,851 & 48 & $5 \%$ \\
\hline All Positions & $\$ 63,452$ & 719 & $\$ 67,786$ & 338 & $\$ 64,838$ & $\mathbf{1 , 0 5 7}$ & $\mathbf{1 0 0} \%$ \\
\hline
\end{tabular}

Excludes Canadian libraries.

Excludes medical and law libraries. 

Canadian ARL University Libraries

TABLES 31-34 
Table 31: Filled Positions; Average, Median, and Beginning Professional Salaries; and Average Years of Professional Experience in Canadian ARL University Libraries, FY 2009-2010

\begin{tabular}{|c|c|c|c|c|c|c|c|c|}
\hline \multirow[b]{2}{*}{ Institution } & \multirow{2}{*}{$\begin{array}{c}\text { Filled } \\
\text { Positions } \\
\text { FY } 2010\end{array}$} & \multicolumn{2}{|c|}{ Average Salaries } & \multicolumn{2}{|c|}{ Median Salaries } & \multicolumn{2}{|c|}{ Beginning Salaries } & \multirow{2}{*}{$\begin{array}{l}\text { Average } \\
\text { Yrs. Exp. } \\
\text { FY } 2010\end{array}$} \\
\hline & & FY 2009 & FY 2010 & FY 2009 & FY 2010 & FY 2009 & FY 2010 & \\
\hline Alberta $^{\ddagger}$ & 62 & $\$ 90,161$ & $\$ 98,203$ & $\$ 96,402$ & $\$ 104,209$ & $\$ 50,632$ & $\$ 50,632$ & 18 \\
\hline British Columbia $^{\ddagger}$ & 94 & 80,717 & 85,196 & 81,585 & 81,675 & 53,557 & 55,335 & 15 \\
\hline Calgary $\ddagger^{\ddagger^{*}}$ & 57 & N/A & 94,823 & N/A & 89,543 & N/A & 58,000 & 18 \\
\hline Guelph $^{\ddagger}$ & 50 & 78,100 & 80,634 & 72,654 & 78,054 & 57,820 & 59,699 & 18 \\
\hline Laval & 62 & 69,203 & 72,293 & 70,607 & 72,888 & 48,094 & 49,547 & 14 \\
\hline McGill & 65 & 73,887 & 74,432 & 67,912 & 67,475 & 50,000 & 50,000 & 17 \\
\hline McMaster ${ }^{\neq}$ & 48 & 79,572 & 71,878 & 79,654 & 72,928 & 45,223 & 46,817 & 17 \\
\hline Manitoba ${ }^{\ddagger}$ & 41 & 90,172 & 95,500 & 94,294 & 100,070 & 47,444 & 48,820 & 23 \\
\hline Montreal $^{\ddagger}$ & 92 & 70,042 & 73,199 & 67,304 & 69,243 & 42,160 & 43,130 & 15 \\
\hline Queen's & 35 & 88,997 & 93,924 & 90,735 & 97,348 & 50,000 & 51,600 & 20 \\
\hline Saskatchewan ${ }^{\ddagger}$ & 40 & 86,263 & 89,027 & 86,845 & 91,994 & 50,101 & 52,731 & 17 \\
\hline Toronto ${ }^{\ddagger}$ & 143 & 91,747 & 91,249 & 92,700 & 87,729 & 51,900 & 51,900 & 16 \\
\hline Waterloo $^{\neq}$ & 36 & 78,214 & 81,938 & 79,102 & 81,972 & 49,586 & 51,804 & 18 \\
\hline Western Ontario $^{\ddagger}$ & 68 & 68,857 & 68,921 & 64,517 & 65,014 & 47,380 & 47,380 & 13 \\
\hline York $^{\ddagger}$ & 54 & 96,722 & 96,016 & 89,352 & 90,210 & 49,000 & 49,000 & 17 \\
\hline
\end{tabular}

Salaries are expressed in Canadian dollars. Directors are included in figures for average years of experience and filled positions, but not in the average and median salary statistics.

Excludes Canadian medical and law libraries. See Tables 35 and 42 for statistics related to medical and law library salaries.

‡ See Footnotes.

* Calgary became a member in FY 2010. 


\section{Table 32: Number and Average Salaries of Canadian ARL University Librarians by Position And Sex, FY 2009-2010}

\begin{tabular}{|c|c|c|c|c|c|c|}
\hline \multirow[b]{2}{*}{ Position } & \multicolumn{2}{|c|}{ WOMEN } & \multicolumn{2}{|c|}{ MeN } & \multicolumn{2}{|c|}{ TOTAL } \\
\hline & Salary & No. & Salary & No. & Salary & No. \\
\hline Director & $\$ 180,043$ & 8 & $\$ 172,996$ & 7 & $\$ 176,755$ & 15 \\
\hline Associate Director & 126,322 & 29 & 130,219 & 11 & 127,393 & 40 \\
\hline Assistant Director & 105,866 & 12 & 108,619 & 7 & 106,880 & 19 \\
\hline Head, Branch & 100,868 & 48 & 103,218 & 12 & 101,338 & 60 \\
\hline Functional Specialist & 75,398 & 85 & 77,961 & 95 & 76,751 & 180 \\
\hline Subject Specialist & 74,508 & 84 & 80,634 & 39 & 76,450 & 123 \\
\hline \multicolumn{7}{|l|}{ Dept. Head: } \\
\hline Acquisitions & 95,750 & 10 & 100,604 & 5 & 97,368 & 15 \\
\hline Reference & $\neq$ & 14 & $\neq$ & 1 & 84,603 & 15 \\
\hline Cataloging & $\neq$ & 9 & $\neq$ & 2 & 93,441 & 11 \\
\hline Serials & 91,840 & 4 & . & & 91,840 & 4 \\
\hline Documents/Maps & $\neq$ & 5 & $\neq$ & 3 & 97,957 & 8 \\
\hline Circulation & $\neq$ & 12 & $\neq$ & 2 & 81,539 & 14 \\
\hline Rare Books/Manuscripts & $\neq$ & 5 & $\ddagger$ & 3 & 97,819 & 8 \\
\hline Computer Systems & $\neq$ & 2 & $\neq$ & 5 & 96,552 & 7 \\
\hline Other & 95,442 & 46 & 91,880 & 21 & 94,325 & 67 \\
\hline \multicolumn{7}{|l|}{ Reference: } \\
\hline Over 14 years experience & 93,618 & 61 & 95,431 & 22 & 94,098 & 83 \\
\hline 10 to 14 years experience & 81,827 & 20 & 83,917 & 9 & 82,476 & 29 \\
\hline 5 to 9 years experience & 72,061 & 53 & 70,493 & 18 & 71,663 & 71 \\
\hline Under 5 years experience & 62,270 & 50 & 67,000 & 11 & 63,123 & 61 \\
\hline \multicolumn{7}{|l|}{ Cataloging: } \\
\hline Over 14 years experience & 86,326 & 23 & 83,761 & 14 & 85,355 & 37 \\
\hline 10 to 14 years experience & $\neq$ & 8 & $\neq$ & 2 & 67,522 & 10 \\
\hline 5 to 9 years experience & $\neq$ & 7 & $\neq$ & 2 & 73,647 & 9 \\
\hline Under 5 years experience & $\neq$ & 7 & $\neq$ & 3 & 62,353 & 10 \\
\hline \multicolumn{7}{|l|}{ Other: } \\
\hline Over 14 years experience & 95,576 & 10 & 82,618 & 7 & 90,241 & 17 \\
\hline 10 to 14 years experience & $\neq$ & 7 & $\neq$ & 3 & 74,179 & 10 \\
\hline 5 to 9 years experience & $\neq$ & 11 & $\neq$ & 1 & 68,674 & 12 \\
\hline Under 5 years experience & $\neq$ & 9 & $\neq$ & 3 & 65,932 & 12 \\
\hline All Positions & $\$ 85,052$ & 639 & $\$ 86,714$ & 308 & $\$ 85,593$ & 947 \\
\hline
\end{tabular}

Salaries are expressed in Canadian dollars.

Excludes Canadian medical and law libraries. See Tables 39 and 46 for salaries in medical and law libraries.

+ Salary data are not published when fewer than four individuals are involved in either category.

. No positions reported in this category. 


\section{Table 33: Number and Average Years of Experience OF CANADIAN ARL UNIVERSITY LibRARIANS by Position and SeX, FY 2009-2010}

\begin{tabular}{|c|c|c|c|c|c|c|}
\hline \multirow[b]{2}{*}{ Position } & \multicolumn{2}{|c|}{ WOMEN } & \multicolumn{2}{|c|}{ MeN } & \multicolumn{2}{|c|}{ Total } \\
\hline & Years & No. & Years & No. & Years & No. \\
\hline Director & 33.5 & 8 & 30.3 & 7 & 32.0 & 15 \\
\hline Associate Director & 23.4 & 29 & 22.8 & 11 & 23.3 & 40 \\
\hline Assistant Director & 23.2 & 12 & 26.0 & 7 & 24.2 & 19 \\
\hline Head, Branch & 21.7 & 48 & 22.9 & 12 & 22.0 & 60 \\
\hline Functional Specialist & 14.3 & 85 & 14.6 & 95 & 14.4 & 180 \\
\hline Subject Specialist & 14.4 & 84 & 15.4 & 39 & 14.7 & 123 \\
\hline \multicolumn{7}{|l|}{ Dept. Head: } \\
\hline Acquisitions & 21.0 & 10 & 21.4 & 5 & 21.1 & 15 \\
\hline Reference & 18.0 & 14 & 31.0 & 1 & 18.9 & 15 \\
\hline Cataloging & 23.9 & 9 & 24.5 & 2 & 24.0 & 11 \\
\hline Serials & 24.0 & 4 & . & & 24.0 & 4 \\
\hline Documents/Maps & 29.6 & 5 & 18.0 & 3 & 25.3 & 8 \\
\hline Circulation & 20.9 & 12 & 5.5 & 2 & 18.7 & 14 \\
\hline Rare Books/Manuscripts & 22.0 & 5 & 27.0 & 3 & 23.9 & 8 \\
\hline Computer Systems & 23.5 & 2 & 19.6 & 5 & 20.7 & 7 \\
\hline Other & 19.5 & 46 & 15.6 & 21 & 18.3 & 67 \\
\hline Public Services & 6.2 & 6 & 8.0 & 4 & 6.9 & 10 \\
\hline Technical Services & 11.5 & 11 & 16.3 & 6 & 13.2 & 17 \\
\hline Administrations & 15.1 & 20 & 17.3 & 4 & 15.4 & 24 \\
\hline Reference & 12.2 & 184 & 14.3 & 60 & 12.7 & 244 \\
\hline Cataloger & 18.3 & 45 & 22.0 & 21 & 19.5 & 66 \\
\hline All Positions & 16.3 & 639 & 16.8 & 308 & 16.5 & 947 \\
\hline
\end{tabular}

Excludes Canadian medical and law libraries. See Tables 40 and 47 for figures in medical and law libraries.

. No positions reported in this category. 
Table 34: Number and Average Salaries

of Canadian ARL University Librarians

by YeARs of ExPERIENCE AND SEX, FY 2009-2010

\begin{tabular}{|c|c|c|c|c|c|c|c|}
\hline \multirow[b]{2}{*}{ Experience } & \multicolumn{2}{|c|}{ WOMEN } & \multicolumn{2}{|c|}{ Men } & \multicolumn{2}{|c|}{ Total } & \multirow{2}{*}{$\begin{array}{c}\% \text { OF } \\
\text { TотAL }\end{array}$} \\
\hline & Salary & No. & Salary & No. & Salary & No. & \\
\hline $0-3$ years & $\$ 61,655$ & 91 & $\$ 65,177$ & 34 & $\$ 62,613$ & 125 & $13 \%$ \\
\hline $4-7$ years & 70,118 & 95 & 68,908 & 40 & 69,760 & 135 & $14 \%$ \\
\hline $8-11$ years & 76,241 & 92 & 76,657 & 46 & 76,380 & 138 & $15 \%$ \\
\hline $12-15$ years & 84,503 & 57 & 79,728 & 30 & 82,856 & 87 & $9 \%$ \\
\hline 16 - 19 years & 87,804 & 57 & 95,036 & 29 & 90,243 & 86 & $9 \%$ \\
\hline $20-23$ years & 93,690 & 67 & 95,595 & 42 & 94,424 & 109 & $12 \%$ \\
\hline $24-27$ years & 101,882 & 48 & 95,053 & 25 & 99,543 & 73 & $8 \%$ \\
\hline $28-31$ years & 103,930 & 42 & 103,164 & 28 & 103,624 & 70 & $7 \%$ \\
\hline $32-35$ years & 99,969 & 43 & 104,388 & 18 & 101,273 & 61 & $6 \%$ \\
\hline over 35 years & 115,100 & 47 & 118,901 & 16 & 116,065 & 63 & $7 \%$ \\
\hline All Positions & $\$ 85,052$ & 639 & $\$ 86,714$ & 308 & $\$ 85,593$ & 947 & $100 \%$ \\
\hline
\end{tabular}

Salaries are expressed in Canadian dollars

Excludes Canadian medical and law libraries. See Tables 41 and 48 for salaries in medical and law libraries.

Note: the "\% of Total" Column in the ARL Annual Salary Survey 2008-2009 listed women's salaries instead of the total percentages. This error has been corrected in this edition. 

ARL University Medical Libraries

TABles 35-41 


\section{Table 35: Filled Positions; Average, Median, Beginning Professional Salaries; and Average Years of Professional Experience \\ in ARL University Medical Libraries, FY 2009-2010}

\begin{tabular}{|c|c|c|c|c|c|}
\hline Institution & $\begin{array}{c}\text { Filled } \\
\text { Positions }\end{array}$ & $\begin{array}{c}\text { Average } \\
\text { Salary }\end{array}$ & $\begin{array}{c}\text { Median } \\
\text { Salary }\end{array}$ & $\begin{array}{c}\text { Beginning } \\
\text { Salary }\end{array}$ & $\begin{array}{l}\text { Average } \\
\text { Yrs. Exp. }\end{array}$ \\
\hline Alabama & 3 & $\neq$ & $\neq$ & $\$ 35,000$ & 11.7 \\
\hline Alberta & 4 & $\neq$ & $\neq$ & 43,398 & 22.5 \\
\hline Arizona & 16 & $\$ 63,771$ & $\$ 58,706$ & 48,605 & 21.1 \\
\hline Boston University & 12 & 52,015 & 47,400 & 43,000 & 7.0 \\
\hline British Columbia & 12 & 70,669 & 74,711 & 47,429 & 17.0 \\
\hline Calgary & 12 & 62,817 & 58,773 & 49,713 & 9.7 \\
\hline California, Davis & 7 & 71,152 & 64,560 & 46,164 & 24.6 \\
\hline California, Irvine & 1 & $\neq$ & $\neq$ & 46,164 & 1.0 \\
\hline California, Los Angeles & 14 & 75,171 & 81,228 & 46,164 & 17.1 \\
\hline California, San Diego & 11 & 68,461 & 68,982 & 46,164 & 13.5 \\
\hline Case Western Reserve & 7 & 59,101 & 62,249 & 35,000 & 26.9 \\
\hline Cincinnati & 17 & 57,738 & 49,004 & 40,000 & 19.7 \\
\hline Columbia & 10 & 68,409 & 70,625 & 51,500 & 16.2 \\
\hline Connecticut & 15 & 76,497 & 75,152 & 55,347 & 16.7 \\
\hline Cornell & 9 & 78,010 & 78,320 & 51,000 & 21.6 \\
\hline Dartmouth & 8 & 62,580 & 60,415 & 45,500 & 19.8 \\
\hline Duke & 20 & 59,342 & 55,479 & 48,000 & 19.0 \\
\hline Emory & 11 & 62,389 & 54,232 & 42,000 & 22.2 \\
\hline Florida & 13 & 57,427 & 54,558 & 42,000 & 14.8 \\
\hline Florida State & 4 & $\neq$ & $\neq$ & 42,000 & 16.3 \\
\hline George Washington & 14 & 64,439 & 64,820 & 48,000 & 15.2 \\
\hline Georgetown & 11 & 59,370 & 61,000 & 44,000 & 12.7 \\
\hline Harvard & 45 & 74,775 & 70,001 & 48,800 & 11.3 \\
\hline Hawaii & 4 & $\neq$ & $\neq$ & 42,660 & 14.5 \\
\hline Howard & 4 & $\neq$ & $\neq$ & 45,000 & 26.0 \\
\hline Illinois, Chicago & 23 & 57,276 & 53,823 & 47,000 & 12.9 \\
\hline Iowa & 10 & 58,987 & 54,540 & 41,000 & 17.0 \\
\hline Johns Hopkins & 25 & 65,575 & 62,644 & 44,000 & 13.8 \\
\hline Kansas & 8 & 52,333 & 49,257 & 38,000 & 15.9 \\
\hline Kentucky & 13 & 57,770 & 57,641 & 41,000 & 24.9 \\
\hline Louisiana State & 1 & $\neq$ & $\neq$ & 36,000 & 4.0 \\
\hline Louisville & 9 & 57,367 & 55,943 & 37,000 & 23.6 \\
\hline McGill & 10 & 61,398 & 55,059 & 42,856 & 13.8 \\
\hline McMaster & 8 & 58,080 & 65,069 & 40,128 & 12.1 \\
\hline Manitoba & 18 & 65,780 & 62,390 & 41,845 & 16.2 \\
\hline Miami & 10 & 71,977 & 72,165 & 50,000 & 20.7 \\
\hline Michigan & 16 & 56,695 & 54,500 & 42,000 & 14.5 \\
\hline Minnesota & 15 & 61,070 & 62,213 & 42,000 & 14.9 \\
\hline
\end{tabular}




\section{Table 35: Filled Positions; Average, Median, Beginning Professional Salaries; and Average Years of Professional Experience \\ in ARL University Medical Libraries, FY 2009-2010}

\begin{tabular}{|c|c|c|c|c|c|}
\hline Institution & $\begin{array}{c}\text { Filled } \\
\text { Positions }\end{array}$ & $\begin{array}{c}\text { Average } \\
\text { Salary }\end{array}$ & $\begin{array}{c}\text { Median } \\
\text { Salary }\end{array}$ & $\begin{array}{c}\text { Beginning } \\
\text { Salary }\end{array}$ & $\begin{array}{l}\text { Average } \\
\text { Yrs. Exp. }\end{array}$ \\
\hline Missouri & 9 & 52,566 & 50,017 & 40,000 & 20.8 \\
\hline Montreal & 10 & 61,382 & 59,349 & 45,332 & 15.1 \\
\hline Nebraska & 15 & 62,725 & 57,200 & 45,000 & 18.9 \\
\hline New Mexico & 16 & 64,016 & 61,309 & 39,372 & 19.4 \\
\hline New York University & 31 & $\$ 65,581$ & $\$ 59,518$ & $\$ 50,000$ & 12.0 \\
\hline North Carolina & 32 & 67,842 & 64,002 & 45,000 & 19.2 \\
\hline Northwestern & 17 & 61,237 & 61,427 & 41,000 & 15.9 \\
\hline Ohio State & 8 & 62,531 & 62,262 & 50,000 & 17.6 \\
\hline Oklahoma & 9 & 59,514 & 60,019 & 35,000 & 18.4 \\
\hline Oklahoma State & 4 & $\neq$ & $\neq$ & 38,000 & 22.0 \\
\hline Pennsylvania & 10 & 63,867 & 60,221 & 43,500 & 20.6 \\
\hline Pennsylvania State & 6 & 63,734 & 61,680 & 42,436 & 17.2 \\
\hline Pittsburgh & 25 & 58,890 & 56,522 & 40,000 & 15.1 \\
\hline Queen`s & 9 & 71,491 & 68,905 & 44,227 & 15.9 \\
\hline Rochester & 25 & 55,936 & 51,703 & 38,168 & 20.0 \\
\hline Saskatchewan & 7 & 62,431 & 59,982 & 45,197 & 9.3 \\
\hline South Carolina & 8 & 50,976 & 47,470 & 34,000 & 14.6 \\
\hline Southern California & 15 & 69,020 & 64,516 & 48,500 & 16.7 \\
\hline Southern Illinois & 6 & 62,521 & 66,592 & 41,000 & 24.5 \\
\hline SUNY Buffalo & 13 & 66,442 & 63,552 & 45,000 & 22.0 \\
\hline SUNY Stony Brook & 19 & 66,198 & 66,191 & 45,000 & 14.7 \\
\hline Temple & 10 & 55,900 & 56,000 & 42,800 & 20.0 \\
\hline Tennessee, Knoxville & 4 & $\neq$ & $\neq$ & 40,000 & 13.0 \\
\hline Tennessee, Memphis & 13 & 54,873 & 51,091 & 45,000 & 22.0 \\
\hline Texas Tech & 22 & 49,585 & 45,389 & 37,000 & 21.0 \\
\hline Toronto & 12 & 80,426 & 90,617 & 44,484 & 17.7 \\
\hline Tulane & 6 & 53,240 & 48,000 & 40,000 & 19.3 \\
\hline Utah & 12 & 63,047 & 59,271 & 38,000 & 20.5 \\
\hline Vanderbilt & 26 & 58,088 & 60,765 & 40,500 & 12.7 \\
\hline Virginia & 13 & 65,396 & 66,000 & 45,000 & 21.9 \\
\hline Washington & 23 & 63,297 & 59,148 & 42,600 & 20.3 \\
\hline Washington U.-St. Louis & 22 & 61,797 & 53,847 & 40,000 & 20.7 \\
\hline Wayne State & 4 & $\neq$ & $\neq$ & 45,000 & 18.3 \\
\hline Wisconsin & 16 & 58,988 & 56,669 & 40,526 & 12.8 \\
\hline Yale & 21 & 75,715 & 73,985 & 50,500 & 17.0 \\
\hline \multicolumn{6}{|c|}{$\begin{array}{l}\text { Directors are included in figures for filled positions and average years of experience, but not in either the average or median salary } \\
\text { statistics. } \\
\text { Canadian salaries are expressed in US dollars. } \\
\text { ‡ Salary data are not published when fewer than four individuals are involved. }\end{array}$} \\
\hline
\end{tabular}




\section{Table 36: Beginning Professional Salaries in ARL University Medical Libraries \\ Rank Order Table, FY 2009-2010}

\begin{tabular}{|c|c|c|c|c|c|}
\hline Rank & Institution & Salary & Rank & Institution & Salary \\
\hline 1 & Connecticut & 55,347 & 38 & McGill & 42,856 \\
\hline 2 & Columbia & 51,500 & 39 & Temple & 42,800 \\
\hline 3 & Cornell & 51,000 & 40 & Hawaii & 42,660 \\
\hline 4 & Yale & 50,500 & 41 & Washington & 42,600 \\
\hline 5 & Miami & 50,000 & 42 & Pennsylvania State & 42,436 \\
\hline 5 & New York & 50,000 & 43 & Emory & 42,000 \\
\hline 5 & Ohio State & 50,000 & 43 & Florida & 42,000 \\
\hline 8 & Calgary & 49,713 & 43 & Florida State & 42,000 \\
\hline 9 & Harvard & 48,800 & 43 & Michigan & 42,000 \\
\hline 10 & Arizona & 48,605 & 43 & Minnesota & 42,000 \\
\hline 11 & Southern California & 48,500 & 48 & Manitoba & 41,845 \\
\hline 12 & Duke & 48,000 & 49 & Iowa & 41,000 \\
\hline 12 & George Washington & 48,000 & 49 & Kentucky & 41,000 \\
\hline 14 & British Columbia & 47,429 & 49 & Northwestern & 41,000 \\
\hline 15 & Illinois, Chicago & 47,000 & 49 & Southern Illinois & 41,000 \\
\hline 16 & Calif. Davis & 46,164 & 53 & Wisconsin & 40,526 \\
\hline 16 & Calif. Irvine & 46,164 & 54 & Vanderbilt & 40,500 \\
\hline 16 & Calif. Los Angeles & 46,164 & 55 & McMaster & 40,128 \\
\hline 16 & Calif. San Diego & 46,164 & 56 & Cincinnati & 40,000 \\
\hline 20 & Dartmouth & 45,500 & 56 & Missouri & 40,000 \\
\hline 21 & Montreal & 45,332 & 56 & Pittsburgh & 40,000 \\
\hline 22 & Saskatchewan & 45,197 & 56 & Tennessee, Knoxville & 40,000 \\
\hline 23 & Howard & 45,000 & 56 & Tulane & 40,000 \\
\hline 23 & Nebraska & 45,000 & 56 & Washington-St. Louis & 40,000 \\
\hline 23 & North Carolina & 45,000 & 62 & New Mexico & 39,372 \\
\hline 23 & SUNY Buffalo & 45,000 & 63 & Rochester & 38,168 \\
\hline 23 & SUNY Stony Brook & 45,000 & 64 & Kansas & 38,000 \\
\hline 23 & Tennessee, Memphis & 45,000 & 64 & Oklahoma State & 38,000 \\
\hline 23 & Virginia & 45,000 & 64 & Utah & 38,000 \\
\hline 23 & Wayne State & 45,000 & 67 & Louisville & 37,000 \\
\hline 31 & Toronto & 44,484 & 67 & Texas Tech & 37,000 \\
\hline 32 & Queen's & 44,227 & 69 & Louisiana State & 36,000 \\
\hline 33 & Georgetown & 44,000 & 70 & Alabama & 35,000 \\
\hline 33 & Johns Hopkins & 44,000 & 70 & Case Western Reserve & 35,000 \\
\hline 35 & Pennsylvania & 43,500 & 70 & Oklahoma & 35,000 \\
\hline 36 & Alberta & 43,398 & 73 & South Carolina & 34,000 \\
\hline 37 & Boston University & 43,000 & & & \\
\hline
\end{tabular}

Beginning salary figures represent officially designated base, not necessarily salaries of actual incumbents.

Canadian salaries are expressed in US dollars. 
Table 37: Median Professional Salaries in ARL University Medical Libraries
Rank Order Table, FY 2009-2010

\begin{tabular}{|c|c|c|c|c|c|}
\hline Rank & Institution & Salary & Rank & Institution & Salary \\
\hline 1 & Toronto & 90,617 & 38 & Washington & 59,148 \\
\hline 2 & California, Los Angeles & 81,228 & 39 & Calgary & 58,773 \\
\hline 3 & Cornell & 78,320 & 40 & Arizona & 58,706 \\
\hline 4 & Connecticut & 75,152 & 41 & Kentucky & 57,641 \\
\hline 5 & British Columbia & 74,711 & 42 & Nebraska & 57,200 \\
\hline 6 & Yale & 73,985 & 43 & Wisconsin & 56,669 \\
\hline 7 & Miami & 72,165 & 44 & Pittsburgh & 56,522 \\
\hline 8 & Columbia & 70,625 & 45 & Temple & 56,000 \\
\hline 9 & Harvard & 70,001 & 46 & Louisville & 55,943 \\
\hline 10 & California, San Diego & 68,982 & 47 & Duke & 55,479 \\
\hline 11 & Queen`s & 68,905 & 48 & McGill & 55,059 \\
\hline 12 & Southern Illinois & 66,592 & 49 & Florida & 54,558 \\
\hline 13 & SUNY Stony Brook & 66,191 & 50 & Iowa & 54,540 \\
\hline 14 & Virginia & 66,000 & 51 & Michigan & 54,500 \\
\hline 15 & McMaster & 65,069 & 52 & Emory & 54,232 \\
\hline 16 & George Washington & 64,820 & 53 & Washington U.-St. Louis & 53,847 \\
\hline 17 & California, Davis & 64,560 & 54 & Illinois, Chicago & 53,823 \\
\hline 18 & Southern California & 64,516 & 55 & Rochester & 51,703 \\
\hline 19 & North Carolina & 64,002 & 56 & Tennessee, Memphis & 51,091 \\
\hline 20 & SUNY Buffalo & 63,552 & 57 & Missouri & 50,017 \\
\hline 21 & Johns Hopkins & 62,644 & 58 & Kansas & 49,257 \\
\hline 22 & Manitoba & 62,390 & 59 & Cincinnati & 49,004 \\
\hline 23 & Ohio State & 62,262 & 60 & Tulane & 48,000 \\
\hline 24 & Case Western Reserve & 62,249 & 61 & South Carolina & 47,470 \\
\hline 25 & Minnesota & 62,213 & 62 & Boston University & 47,400 \\
\hline 26 & Pennsylvania State & 61,680 & 63 & Texas Tech & 45,389 \\
\hline 27 & Northwestern & 61,427 & & Alabama & * \\
\hline 28 & New Mexico & 61,309 & & Alberta & * \\
\hline 29 & Georgetown & 61,000 & & California, Irvine & * \\
\hline 30 & Vanderbilt & 60,765 & & Florida State & * \\
\hline 31 & Dartmouth & 60,415 & & Hawaii & * \\
\hline 32 & Pennsylvania & 60,221 & & Howard & * \\
\hline 33 & Oklahoma & 60,019 & & Louisiana State & * \\
\hline 34 & Saskatchewan & 59,982 & & Oklahoma State & * \\
\hline 35 & New York University & 59,518 & & Tennessee, Knoxville & * \\
\hline 36 & Montreal & 59,349 & & Wayne State & * \\
\hline 37 & Utah & 59,271 & & & \\
\hline
\end{tabular}

Salaries of directors are not included in the calculation of medians. Alabama, Alberta, California-Irvine, Florida State, Hawaii, Howard, Louisiana State, Oklahoma State, Tennessee-Knoxville, and Wayne State are not ranked because they reported fewer than four individuals. Canadian salaries are expressed in US dollars. 
Table 38: Average Professional Salaries in ARL University Medical Libraries

RANK ORder TABLE, FY 2009-2010

\begin{tabular}{|c|c|c|c|c|c|}
\hline Rank & Institution & Salary & Rank & Institution & Salary \\
\hline 1 & Toronto & 80,426 & 38 & Northwestern & 61,237 \\
\hline 2 & Cornell & 78,010 & 39 & Minnesota & 61,070 \\
\hline 3 & Connecticut & 76,497 & 40 & Oklahoma & 59,514 \\
\hline 4 & Yale & 75,715 & 41 & Georgetown & 59,370 \\
\hline 5 & California, Los Angeles & 75,171 & 42 & Duke & 59,342 \\
\hline 6 & Harvard & 74,775 & 43 & Case Western Reserve & 59,101 \\
\hline 7 & Miami & 71,977 & 44 & Wisconsin & 58,988 \\
\hline 8 & Queen`s & 71,491 & 45 & Iowa & 58,987 \\
\hline 9 & California, Davis & 71,152 & 46 & Pittsburgh & 58,890 \\
\hline 10 & British Columbia & 70,669 & 47 & Vanderbilt & 58,088 \\
\hline 11 & Southern California & 69,020 & 48 & McMaster & 58,080 \\
\hline 12 & California, San Diego & 68,461 & 49 & Kentucky & 57,770 \\
\hline 13 & Columbia & 68,409 & 50 & Cincinnati & 57,738 \\
\hline 14 & North Carolina & 67,842 & 51 & Florida & 57,427 \\
\hline 15 & SUNY Buffalo & 66,442 & 52 & Louisville & 57,367 \\
\hline 16 & SUNY Stony Brook & 66,198 & 53 & Illinois, Chicago & 57,276 \\
\hline 17 & Manitoba & 65,780 & 54 & Michigan & 56,695 \\
\hline 18 & New York University & 65,581 & 55 & Rochester & 55,936 \\
\hline 19 & Johns Hopkins & 65,575 & 56 & Temple & 55,900 \\
\hline 20 & Virginia & 65,396 & 57 & Tennessee, Memphis & 54,873 \\
\hline 21 & George Washington & 64,439 & 58 & Tulane & 53,240 \\
\hline 22 & New Mexico & 64,016 & 59 & Missouri & 52,566 \\
\hline 23 & Pennsylvania & 63,867 & 60 & Kansas & 52,333 \\
\hline 24 & Arizona & 63,771 & 61 & Boston University & 52,015 \\
\hline 25 & Pennsylvania State & 63,734 & 62 & South Carolina & 50,976 \\
\hline 26 & Washington & 63,297 & 63 & Texas Tech & 49,585 \\
\hline 27 & Utah & 63,047 & & Alabama & * \\
\hline 28 & Calgary & 62,817 & & Alberta & * \\
\hline 29 & Nebraska & 62,725 & & California, Irvine & * \\
\hline 30 & Dartmouth & 62,580 & & Florida State & * \\
\hline 31 & Ohio State & 62,531 & & Hawaii & * \\
\hline 32 & Southern Illinois & 62,521 & & Howard & * \\
\hline 33 & Saskatchewan & 62,431 & & Louisiana State & * \\
\hline 34 & Emory & 62,389 & & Oklahoma State & * \\
\hline 35 & Washington U.-St. Louis & 61,797 & & Tennessee, Knoxville & * \\
\hline 36 & McGill & 61,398 & & Wayne State & * \\
\hline 37 & Montreal & 61,382 & & & \\
\hline
\end{tabular}

Salaries of directors are not included in the calculation of medians. Alabama, Alberta, California-Irvine, Florida State, Hawaii, Howard, Louisiana State, Oklahoma State, Tennessee-Knoxville, and Wayne State are not ranked because they reported fewer than four individuals. Canadian salaries are expressed in US dollars. 


\section{Table 39: Number and Average Salaries of ARL University Medical Librarians by Position AND SEX, FY 2009-2010}

\begin{tabular}{|c|c|c|c|c|c|c|}
\hline \multirow[b]{2}{*}{ Position } & \multicolumn{2}{|c|}{ WOMEN } & \multicolumn{2}{|c|}{ MeN } & \multicolumn{2}{|c|}{ TOTAL } \\
\hline & Salary & No. & Salary & No. & Salary & No. \\
\hline Head, Medical & $\$ 121,618$ & 49 & $\$ 129,622$ & 19 & $\$ 123,855$ & 68 \\
\hline Associate Director & 85,716 & 33 & 94,463 & 11 & 87,903 & 44 \\
\hline Assistant Director & 67,564 & 33 & 64,503 & 7 & 67,028 & 40 \\
\hline Head, Branch & 63,688 & 26 & $\cdot$ & & 63,688 & 26 \\
\hline Functional Specialist & 60,366 & 73 & 62,517 & 91 & 61,559 & 164 \\
\hline Subject Specialist & 60,258 & 65 & 59,940 & 10 & 60,215 & 75 \\
\hline \multicolumn{7}{|l|}{ Dept. Head: } \\
\hline Acquisitions & 67,709 & 16 & 64,299 & 4 & 67,027 & 20 \\
\hline Reference & 67,878 & 17 & 78,374 & 7 & 70,940 & 24 \\
\hline Cataloging & $\neq$ & 10 & $\neq$ & 1 & 65,279 & 11 \\
\hline Serials & $\neq$ & 6 & $\ddagger$ & 2 & 54,269 & 8 \\
\hline Documents/Maps & $\neq$ & 1 & . & & $\neq$ & 1 \\
\hline Circulation & 59,032 & 11 & 62,983 & 8 & 60,696 & 19 \\
\hline Rare Books/Manuscripts & $\neq$ & 2 & $\neq$ & 6 & 70,824 & 8 \\
\hline Computer Systems & 78,337 & 5 & 87,697 & 9 & 84,354 & 14 \\
\hline Other & 66,866 & 50 & 72,008 & 16 & 68,112 & 66 \\
\hline \multicolumn{7}{|l|}{ Reference: } \\
\hline Over 14 years experience & 64,369 & 94 & 65,642 & 18 & 64,574 & 112 \\
\hline 10 to 14 years experience & 61,674 & 27 & 65,117 & 8 & 62,461 & 35 \\
\hline 5 to 9 years experience & 55,685 & 41 & 58,381 & 13 & 56,334 & 54 \\
\hline Under 5 years experience & 48,240 & 41 & 46,875 & 6 & 48,066 & 47 \\
\hline \multicolumn{7}{|l|}{ Cataloging: } \\
\hline Over 14 years experience & $\neq$ & 6 & $\neq$ & 2 & 62,813 & 8 \\
\hline 10 to 14 years experience & $\neq$ & 2 & $\cdot$ & & $\neq$ & 2 \\
\hline 5 to 9 years experience & 50,278 & 5 & . & & 50,278 & 5 \\
\hline Under 5 years experience & $\neq$ & 2 & & & $\neq$ & 2 \\
\hline \multicolumn{7}{|l|}{ Other: } \\
\hline Over 14 years experience & 62,322 & 32 & 62,444 & 10 & 62,351 & 42 \\
\hline 10 to 14 years experience & $\neq$ & 11 & $\neq$ & 3 & 58,211 & 14 \\
\hline 5 to 9 years experience & $\neq$ & 16 & $\neq$ & 3 & 55,301 & 19 \\
\hline Under 5 years experience & 45,239 & 12 & 44,788 & 8 & 45,059 & 20 \\
\hline All Positions & $\$ 66,583$ & 686 & $\$ 69,796$ & 262 & $\$ 67,471$ & 948 \\
\hline
\end{tabular}

Canadian salaries are expressed in US dollars.

₹ Salary data are not published when fewer than four individuals are involved in either category.

. No positions reported in this category. 
Table 40: Number and Average Years of Experience of ARL University Medical Librarians by Position And Sex, FY 2009-2010

\begin{tabular}{|c|c|c|c|c|c|c|}
\hline \multirow[b]{2}{*}{ Position } & \multicolumn{2}{|c|}{ WOMEN } & \multicolumn{2}{|c|}{ Men } & \multicolumn{2}{|c|}{ TOTAL } \\
\hline & Years & No. & Years & No. & Years & No. \\
\hline Head, Medical & 29.8 & 49 & 29.1 & 19 & 29.6 & 68 \\
\hline Associate Director & 25.7 & 33 & 25.4 & 11 & 25.6 & 44 \\
\hline Assistant Director & 20.3 & 33 & 13.0 & 7 & 19.0 & 40 \\
\hline Head, Branch & 18.3 & 26 & . & & 18.3 & 26 \\
\hline Functional Specialist & 14.2 & 73 & 11.5 & 91 & 12.7 & 164 \\
\hline Subject Specialist & 15.8 & 65 & 12.7 & 10 & 15.4 & 75 \\
\hline \multicolumn{7}{|l|}{ Dept. Head: } \\
\hline Acquisitions & 20.4 & 16 & 15.3 & 4 & 19.4 & 20 \\
\hline Reference & 21.2 & 17 & 22.6 & 7 & 21.6 & 24 \\
\hline Cataloging & 19.5 & 10 & 33.0 & 1 & 20.7 & 11 \\
\hline Serials & 16.2 & 6 & 14.5 & 2 & 15.8 & 8 \\
\hline Documents/Maps & 19.0 & 1 & . & & 19.0 & 1 \\
\hline Circulation & 20.5 & 11 & 8.6 & 8 & 15.5 & 19 \\
\hline Rare Books/Manuscripts & 29.0 & 2 & 24.2 & 6 & 25.4 & 8 \\
\hline Computer Systems & 16.0 & 5 & 18.6 & 9 & 17.6 & 14 \\
\hline Other & 18.5 & 50 & 13.8 & 16 & 17.3 & 66 \\
\hline Public Services & 14.8 & 44 & 11.3 & 16 & 13.8 & 60 \\
\hline Technical Services & 16.7 & 15 & 19.3 & 4 & 17.3 & 19 \\
\hline Administrative Services & 16.3 & 12 & 14.3 & 4 & 15.8 & 16 \\
\hline Reference & 15.0 & 203 & 14.1 & 45 & 14.8 & 248 \\
\hline Cataloger & 12.4 & 15 & 26.0 & 2 & 14.0 & 17 \\
\hline All Positions & 17.7 & 686 & 15.2 & 262 & 17.0 & 948 \\
\hline
\end{tabular}

. No positions reported in this category. 
Table 41: Number and Average Salaries of ARL University Medical Librarians by YeARS OF EXPERIENCE AND SEX, FY 2009-2010

\begin{tabular}{|c|c|c|c|c|c|c|c|}
\hline \multirow[b]{2}{*}{ Experience } & \multicolumn{2}{|c|}{ WOMEN } & \multicolumn{2}{|c|}{ MeN } & \multicolumn{2}{|c|}{ Total } & \multirow{2}{*}{$\begin{array}{l}\% \text { OF } \\
\text { TotaL }\end{array}$} \\
\hline & Salary & No. & Salary & No. & Salary & No. & \\
\hline $0-3$ years & $\$ 49,526$ & 72 & $\$ 51,643$ & 39 & $\$ 50,270$ & 111 & $12 \%$ \\
\hline $4-7$ years & 53,948 & 86 & 55,351 & 34 & 54,345 & 120 & $13 \%$ \\
\hline $8-11$ years & 59,471 & 103 & 66,052 & 45 & 61,472 & 148 & $16 \%$ \\
\hline $12-15$ years & 67,416 & 66 & 67,047 & 39 & 67,279 & 105 & $11 \%$ \\
\hline $16-19$ years & 64,403 & 68 & 77,676 & 19 & 67,301 & 87 & $9 \%$ \\
\hline $20-23$ years & 66,349 & 52 & 82,710 & 21 & 71,056 & 73 & $8 \%$ \\
\hline $24-27$ years & 72,372 & 69 & 77,918 & 18 & 73,520 & 87 & $9 \%$ \\
\hline 28 - 31 years & 75,216 & 75 & 82,278 & 22 & 76,818 & 97 & $10 \%$ \\
\hline $32-35$ years & 87,271 & 54 & 94,280 & 17 & 88,949 & 71 & $7 \%$ \\
\hline over 35 years & 90,704 & 41 & 96,915 & 8 & 91,718 & 49 & $5 \%$ \\
\hline All Positions & $\$ 66,583$ & 686 & $\$ 69,796$ & 262 & $\$ 67,471$ & 948 & $100 \%$ \\
\hline
\end{tabular}

Canadian salaries are expressed in US dollars. 

ARL UNIVERSITY LAW LIBRARIES

TABLES $42-48$ 


\section{Table 42: Filled Positions; Average, Median, Beginning Professional Salaries; and Average Years of Experience \\ in ARL University LaW Libraries, FY 2009-2010}

\begin{tabular}{|c|c|c|c|c|c|}
\hline Institution & $\begin{array}{c}\text { Filled } \\
\text { Positions }\end{array}$ & $\begin{array}{c}\text { Average } \\
\text { Salary }\end{array}$ & $\begin{array}{c}\text { Median } \\
\text { Salary }\end{array}$ & $\begin{array}{c}\text { Beginning } \\
\text { Salary }\end{array}$ & $\begin{array}{l}\text { Average } \\
\text { Yrs. Exp. }\end{array}$ \\
\hline Alabama & 10 & $\$ 59,066$ & $\$ 57,320$ & $\$ 52,000$ & 19.3 \\
\hline Alberta & 4 & $\neq$ & $\neq$ & 45,594 & 28.5 \\
\hline Arizona & 10 & 63,849 & 65,000 & 50,000 & 14.8 \\
\hline Arizona State & 7 & 65,701 & 66,584 & 45,000 & 21.6 \\
\hline Boston University & 11 & 70,830 & 64,300 & 55,000 & 16.8 \\
\hline Boston College & 14 & 74,773 & 77,625 & 50,000 & 19.1 \\
\hline British Columbia & 3 & $\neq$ & $\neq$ & 47,429 & 23.3 \\
\hline Calgary & 3 & $\neq$ & $\neq$ & 49,713 & 18.0 \\
\hline California, Davis & 8 & 73,380 & 68,892 & 46,164 & 17.9 \\
\hline California, Irvine & 1 & $\neq$ & $\neq$ & 46,164 & 9.0 \\
\hline California, Los Angeles & 15 & 78,860 & 84,455 & 46,164 & 14.1 \\
\hline Case Western Reserve & 12 & 67,090 & 66,149 & 35,000 & 19.3 \\
\hline Cincinnati & 6 & 64,155 & 53,580 & 44,000 & 22.2 \\
\hline Colorado & 8 & 72,313 & 59,901 & 45,000 & 17.9 \\
\hline Columbia & 18 & 71,168 & 61,116 & 51,500 & 11.7 \\
\hline Connecticut & 11 & 75,922 & 74,684 & 41,857 & 18.6 \\
\hline Cornell & 7 & 79,220 & 73,015 & 58,000 & 15.6 \\
\hline Duke & 11 & 74,075 & 68,575 & 55,000 & 17.8 \\
\hline Emory & 9 & 58,084 & 52,941 & 44,000 & 12.9 \\
\hline Florida & 9 & 61,129 & 57,699 & 50,000 & 17.2 \\
\hline Florida State & 10 & 55,938 & 53,494 & 52,000 & 20.0 \\
\hline George Washington & 22 & 85,686 & 80,007 & 58,000 & 13.9 \\
\hline Georgetown & 25 & 74,865 & 70,000 & 50,000 & 11.5 \\
\hline Georgia & 8 & 60,001 & 56,901 & 40,000 & 17.0 \\
\hline Harvard & 39 & 73,096 & 68,525 & 48,800 & 14.6 \\
\hline Hawaii & 5 & 84,551 & 81,193 & 55,000 & 15.6 \\
\hline Houston & 12 & 54,915 & 51,500 & 51,000 & 16.0 \\
\hline Howard & 6 & 48,423 & 49,914 & 45,000 & 20.7 \\
\hline Illinois, Urbana & 10 & 61,284 & 59,129 & 54,000 & 15.5 \\
\hline Indiana & 10 & 69,325 & 64,923 & 40,400 & 21.0 \\
\hline Iowa & 17 & 76,488 & 70,750 & 41,000 & 21.3 \\
\hline Kansas & 8 & 49,563 & 45,297 & 38,000 & 9.6 \\
\hline Kentucky & 7 & 52,015 & 47,838 & 41,000 & 11.6 \\
\hline Louisiana State & 9 & 57,380 & 55,846 & 40,000 & 17.2 \\
\hline Louisville & 6 & 59,853 & 59,931 & 37,000 & 19.3 \\
\hline McGill & 4 & $\neq$ & $\neq$ & 42,856 & 16.5 \\
\hline Manitoba & 3 & $\neq$ & $\neq$ & 41,851 & 28.3 \\
\hline Miami & 14 & 56,251 & 53,000 & 45,000 & 15.7 \\
\hline Michigan & 12 & 78,454 & 70,553 & 48,000 & 15.8 \\
\hline Minnesota & 14 & 75,686 & 68,200 & 46,000 & 20.0 \\
\hline
\end{tabular}




\section{Table 42: Filled Positions; Average, Median, Beginning Professional Salaries; and Average Years of Experience \\ in ARL University LaW Libraries, FY 2009-2010}

\begin{tabular}{|c|c|c|c|c|c|}
\hline Institution & $\begin{array}{c}\text { Filled } \\
\text { Positions }\end{array}$ & $\begin{array}{c}\text { Average } \\
\text { Salary }\end{array}$ & $\begin{array}{c}\text { Median } \\
\text { Salary }\end{array}$ & $\begin{array}{c}\text { Beginning } \\
\text { Salary }\end{array}$ & $\begin{array}{l}\text { Average } \\
\text { Yrs. Exp. }\end{array}$ \\
\hline Missouri & 8 & 54,645 & 57,327 & 40,000 & 13.6 \\
\hline Montreal & 5 & 60,353 & 55,200 & 45,332 & 10.8 \\
\hline Nebraska & 5 & 63,975 & 63,772 & 45,000 & 17.4 \\
\hline New Mexico & 7 & 63,420 & 56,928 & 50,000 & 9.3 \\
\hline New York University & 19 & 79,659 & 79,673 & 60,000 & 21.6 \\
\hline North Carolina & 12 & 74,947 & 68,000 & 45,000 & 15.8 \\
\hline Northwestern & 11 & 63,154 & 59,572 & 44,000 & 19.5 \\
\hline Notre Dame & 13 & 71,539 & 66,248 & 41,200 & 18.4 \\
\hline Ohio State & 7 & 68,558 & 63,173 & 42,000 & 13.6 \\
\hline Oklahoma & 7 & 57,410 & 51,269 & 42,000 & 14.7 \\
\hline Oregon & 6 & 56,465 & 57,846 & 42,000 & 20.8 \\
\hline Pennsylvania & 15 & 70,502 & 63,865 & 43,500 & 17.3 \\
\hline Pennsylvania State & 10 & 75,080 & 69,360 & 55,000 & 20.3 \\
\hline Queen`s & 3 & $\neq$ & $\neq$ & 44,227 & 9.7 \\
\hline Rutgers, Camden & 8 & 84,946 & 87,500 & 60,000 & 22.0 \\
\hline Rutgers, Newark & 10 & 72,346 & 64,000 & 52,000 & 16.5 \\
\hline Saskatchewan & 3 & $\neq$ & $\neq$ & 45,197 & 22.0 \\
\hline South Carolina & 8 & 63,808 & 67,619 & 50,000 & 9.3 \\
\hline Southern Illinois & 4 & $\neq$ & $\neq$ & 50,000 & 8.5 \\
\hline SUNY Buffalo & 10 & 69,905 & 77,137 & 45,000 & 17.9 \\
\hline Syracuse & 9 & 57,805 & 54,353 & 47,000 & 15.0 \\
\hline Temple & 10 & 61,988 & 51,654 & 42,448 & 23.1 \\
\hline Tennessee & 8 & 70,669 & 59,440 & 50,000 & 18.0 \\
\hline Texas & 15 & 58,876 & 53,857 & 38,000 & 16.3 \\
\hline Texas Tech & 8 & 63,439 & 65,549 & 47,000 & 13.3 \\
\hline Toronto & 6 & 74,123 & 73,979 & 44,484 & 17.8 \\
\hline Tulane & 7 & 58,420 & 56,970 & 40,000 & 17.6 \\
\hline Utah & 8 & 57,095 & 52,530 & 41,500 & 16.5 \\
\hline Vanderbilt & 5 & 68,154 & 59,830 & 40,500 & 20.0 \\
\hline Virginia & 14 & 68,146 & 63,500 & 63,500 & 15.5 \\
\hline Washington & 16 & 72,614 & 70,020 & 59,000 & 22.1 \\
\hline Washington U.-St. Louis & 10 & 62,335 & 57,500 & 50,000 & 18.5 \\
\hline Wayne State & 3 & $\neq$ & $\neq$ & 45,000 & 19.3 \\
\hline Western Ontario & 3 & $\neq$ & $\neq$ & 40,610 & 14.3 \\
\hline Wisconsin & 12 & 62,919 & 58,954 & 40,526 & 23.1 \\
\hline Yale & 19 & 81,322 & 81,500 & 50,500 & 18.7 \\
\hline York & 5 & 71,436 & 72,376 & 41,999 & 15.2 \\
\hline \multicolumn{6}{|c|}{$\begin{array}{l}\text { Directors are included in figures for filled positions and average years of experience, but not in either the average or median salary } \\
\text { statistics. } \\
\text { Canadian salaries are expressed in US dollars. } \\
\text { ‡ Salary data are not published when fewer than four individuals are involved. }\end{array}$} \\
\hline
\end{tabular}


Table 43: Beginning Professional Salaries in ARL University Law Libraries

Rank Order Table, FY 2009-2010

\begin{tabular}{|c|c|c|c|c|c|}
\hline Rank & Institution & Salary & Rank & Institution & Salary \\
\hline 1 & Virginia & 63,500 & 40 & Arizona State & 45,000 \\
\hline 2 & New York & 60,000 & 40 & Colorado & 45,000 \\
\hline 2 & Rutgers, Camden Law & 60,000 & 40 & Howard & 45,000 \\
\hline 4 & Washington & 59,000 & 40 & Miami & 45,000 \\
\hline 5 & Cornell & 58,000 & 40 & Nebraska & 45,000 \\
\hline 5 & George Washington & 58,000 & 40 & North Carolina & 45,000 \\
\hline 7 & Boston University & 55,000 & 40 & SUNY Buffalo & 45,000 \\
\hline 7 & Duke & 55,000 & 40 & Wayne State & 45,000 \\
\hline 7 & Hawaii & 55,000 & 48 & Toronto & 44,484 \\
\hline 7 & Pennsylvania State & 55,000 & 49 & Queen's & 44,227 \\
\hline 11 & Illinois, Urbana & 54,000 & 50 & Cincinnati & 44,000 \\
\hline 12 & Alabama & 52,000 & 50 & Emory & 44,000 \\
\hline 12 & Florida State & 52,000 & 50 & Northwestern & 44,000 \\
\hline 12 & Rutgers, Newark Law & 52,000 & 53 & Pennsylvania & 43,500 \\
\hline 15 & Columbia & 51,500 & 54 & McGill & 42,856 \\
\hline 16 & Houston & 51,000 & 55 & Temple & 42,448 \\
\hline 17 & Yale & 50,500 & 56 & Ohio State & 42,000 \\
\hline 18 & Arizona & 50,000 & 56 & Oklahoma & 42,000 \\
\hline 18 & Boston College & 50,000 & 56 & Oregon & 42,000 \\
\hline 18 & Florida & 50,000 & 59 & York & 41,999 \\
\hline 18 & Georgetown & 50,000 & 60 & Connecticut & 41,857 \\
\hline 18 & New Mexico & 50,000 & 61 & Manitoba & 41,851 \\
\hline 18 & South Carolina & 50,000 & 62 & Utah & 41,500 \\
\hline 18 & Southern Illinois & 50,000 & 63 & Notre Dame & 41,200 \\
\hline 18 & Tennessee & 50,000 & 64 & Iowa & 41,000 \\
\hline 18 & Washington-St. Louis & 50,000 & 64 & Kentucky & 41,000 \\
\hline 27 & Calgary & 49,713 & 66 & Western Ontario & 40,610 \\
\hline 28 & Harvard & 48,800 & 67 & Wisconsin & 40,526 \\
\hline 29 & Michigan & 48,000 & 68 & Vanderbilt & 40,500 \\
\hline 30 & British Columbia & 47,429 & 69 & Indiana & 40,400 \\
\hline 31 & Syracuse & 47,000 & 70 & Georgia & 40,000 \\
\hline 31 & Texas Tech & 47,000 & 70 & Louisiana State & 40,000 \\
\hline 33 & Calif. Davis & 46,164 & 70 & Missouri & 40,000 \\
\hline 33 & Calif. Irvine & 46,164 & 70 & Tulane & 40,000 \\
\hline 33 & Calif. Los Angeles & 46,164 & 74 & Kansas & 38,000 \\
\hline 36 & Minnesota & 46,000 & 74 & Texas & 38,000 \\
\hline 37 & Alberta & 45,594 & 76 & Louisville & 37,000 \\
\hline 38 & Montreal & 45,332 & 77 & Case Western Reserve & 35,000 \\
\hline 39 & Saskatchewan & 7 & & & \\
\hline
\end{tabular}

Beginning salary figures represent officially designated base, not necessarily salaries of actual incumbents.

Canadian salaries are expressed in US dollars. 


\section{Table 44: Median Professional Salaries in ARL University Law Libraries \\ RanK ORder Table, FY 2009-2010}

\begin{tabular}{|c|c|c|c|c|c|}
\hline Rank & Institution & Salary & Rank & Institution & Salary \\
\hline 1 & Rutgers, Camden & 87,500 & 39 & Vanderbilt & 59,830 \\
\hline 2 & California, Los Angeles & 84,455 & 40 & Northwestern & 59,572 \\
\hline 3 & Yale & 81,500 & 41 & Tennessee & 59,440 \\
\hline 4 & Hawaii & 81,193 & 42 & Illinois, Urbana & 59,129 \\
\hline 5 & George Washington & 80,007 & 43 & Wisconsin & 58,954 \\
\hline 6 & New York University & 79,673 & 44 & Oregon & 57,846 \\
\hline 7 & Boston College & 77,625 & 45 & Florida & 57,699 \\
\hline 8 & SUNY Buffalo & 77,137 & 46 & Washington U.-St. Louis & 57,500 \\
\hline 9 & Connecticut & 74,684 & 47 & Missouri & 57,327 \\
\hline 10 & Toronto & 73,979 & 48 & Alabama & 57,320 \\
\hline 11 & Cornell & 73,015 & 49 & Tulane & 56,970 \\
\hline 12 & York & 72,376 & 50 & New Mexico & 56,928 \\
\hline 13 & Iowa & 70,750 & 51 & Georgia & 56,901 \\
\hline 14 & Michigan & 70,553 & 52 & Louisiana State & 55,846 \\
\hline 15 & Washington & 70,020 & 53 & Montreal & 55,200 \\
\hline 16 & Georgetown & 70,000 & 54 & Syracuse & 54,353 \\
\hline 17 & Pennsylvania State & 69,360 & 55 & Texas & 53,857 \\
\hline 18 & California, Davis & 68,892 & 56 & Cincinnati & 53,580 \\
\hline 19 & Duke & 68,575 & 57 & Florida State & 53,494 \\
\hline 20 & Harvard & 68,525 & 58 & Miami & 53,000 \\
\hline 21 & Minnesota & 68,200 & 59 & Emory & 52,941 \\
\hline 22 & North Carolina & 68,000 & 60 & Utah & 52,530 \\
\hline 23 & South Carolina & 67,619 & 61 & Temple & 51,654 \\
\hline 24 & Arizona State & 66,584 & 62 & Houston & 51,500 \\
\hline 25 & Notre Dame & 66,248 & 63 & Oklahoma & 51,269 \\
\hline 26 & Case Western Reserve & 66,149 & 64 & Howard & 49,914 \\
\hline 27 & Texas Tech & 65,549 & 65 & Kentucky & 47,838 \\
\hline 28 & Arizona & 65,000 & 66 & Kansas & 45,297 \\
\hline 29 & Indiana & 64,923 & & Alberta & * \\
\hline 30 & Boston University & 64,300 & & British Columbia & * \\
\hline 31 & Rutgers, Newark & 64,000 & & Calgary & * \\
\hline 32 & Pennsylvania & 63,865 & & McGill & * \\
\hline 33 & Nebraska & 63,772 & & Manitoba & * \\
\hline 34 & Virginia & 63,500 & & Queen`s & * \\
\hline 35 & Ohio State & 63,173 & & Saskatchewan & * \\
\hline 36 & Columbia & 61,116 & & Southern Illinois & * \\
\hline 37 & Louisville & 59,931 & & Wayne State & * \\
\hline 38 & Colorado & 59,901 & & Western Ontario & * \\
\hline
\end{tabular}

Salaries of directors are not included in the calculation of medians. Alberta, British Columbia, Calgary, McGill, Manitoba, Queen's, Saskatchewan, Southern Illinois, Wayne State, and Western Ontario are not ranked because they reported fewer than four individuals. Canadian salaries are expressed in US dollars. 
Table 45: Average Professional Salaries in ARL University Law Libraries
Rank Order Table, FY 2009-2010

\begin{tabular}{|c|c|c|c|c|c|}
\hline Rank & Institution & Salary & Rank & Institution & Salary \\
\hline 1 & George Washington & 85,686 & 39 & South Carolina & 63,808 \\
\hline 2 & Rutgers, Camden & 84,946 & 40 & Texas Tech & 63,439 \\
\hline 3 & Hawaii & 84,551 & 41 & New Mexico & 63,420 \\
\hline 4 & Yale & 81,322 & 42 & Northwestern & 63,154 \\
\hline 5 & New York University & 79,659 & 43 & Wisconsin & 62,919 \\
\hline 6 & Cornell & 79,220 & 44 & Washington U.-St. Louis & 62,335 \\
\hline 7 & California, Los Angeles & 78,860 & 45 & Temple & 61,988 \\
\hline 8 & Michigan & 78,454 & 46 & Illinois, Urbana & 61,284 \\
\hline 9 & Iowa & 76,488 & 47 & Florida & 61,129 \\
\hline 10 & Connecticut & 75,922 & 48 & Montreal & 60,353 \\
\hline 11 & Minnesota & 75,686 & 49 & Georgia & 60,001 \\
\hline 12 & Pennsylvania State & 75,080 & 50 & Louisville & 59,853 \\
\hline 13 & North Carolina & 74,947 & 51 & Alabama & 59,066 \\
\hline 14 & Georgetown & 74,865 & 52 & Texas & 58,876 \\
\hline 15 & Boston College & 74,773 & 53 & Tulane & 58,420 \\
\hline 16 & Toronto & 74,123 & 54 & Emory & 58,084 \\
\hline 17 & Duke & 74,075 & 55 & Syracuse & 57,805 \\
\hline 18 & California, Davis & 73,380 & 56 & Oklahoma & 57,410 \\
\hline 19 & Harvard & 73,096 & 57 & Louisiana State & 57,380 \\
\hline 20 & Washington & 72,614 & 58 & Utah & 57,095 \\
\hline 21 & Rutgers, Newark & 72,346 & 59 & Oregon & 56,465 \\
\hline 22 & Colorado & 72,313 & 60 & Miami & 56,251 \\
\hline 23 & Notre Dame & 71,539 & 61 & Florida State & 55,938 \\
\hline 24 & York & 71,436 & 62 & Houston & 54,915 \\
\hline 25 & Columbia & 71,168 & 63 & Missouri & 54,645 \\
\hline 26 & Boston University & 70,830 & 64 & Kentucky & 52,015 \\
\hline 27 & Tennessee & 70,669 & 65 & Kansas & 49,563 \\
\hline 28 & Pennsylvania & 70,502 & 66 & Howard & 48,423 \\
\hline 29 & SUNY Buffalo & 69,905 & & Alberta & * \\
\hline 30 & Indiana & 69,325 & & British Columbia & * \\
\hline 31 & Ohio State & 68,558 & & Calgary & * \\
\hline 32 & Vanderbilt & 68,154 & & McGill & * \\
\hline 33 & Virginia & 68,146 & & Manitoba & * \\
\hline 34 & Case Western Reserve & 67,090 & & Queen`s & * \\
\hline 35 & Arizona State & 65,701 & & Saskatchewan & * \\
\hline 36 & Cincinnati & 64,155 & & Southern Illinois & * \\
\hline 37 & Nebraska & 63,975 & & Wayne State & * \\
\hline 38 & Arizona & 63,849 & & Western Ontario & * \\
\hline
\end{tabular}

Salaries of directors are not included in the calculation of medians. Alberta, British Columbia, Calgary, McGill, Manitoba, Queen's, Saskatchewan, Southern Illinois, Wayne State, and Western Ontario are not ranked because they reported fewer than four individuals. Canadian salaries are expressed in US dollars. 


\section{Table 46: Number and Average Salaries of ARL University LaW Librarians by Position AND Sex, FY 2009-2010}

\begin{tabular}{|c|c|c|c|c|c|c|}
\hline \multirow[b]{2}{*}{ Position } & \multicolumn{2}{|c|}{ WOMEN } & \multicolumn{2}{|c|}{ MeN } & \multicolumn{2}{|c|}{ Total } \\
\hline & Salary & No. & Salary & No. & Salary & No. \\
\hline Head, Law & $\$ 151,028$ & 39 & $\$ 155,267$ & 38 & $\$ 153,120$ & 77 \\
\hline Associate Director & 93,011 & 34 & 93,519 & 24 & 93,221 & 58 \\
\hline Assistant Director & 90,055 & 24 & 89,016 & 10 & 89,749 & 34 \\
\hline Functional Specialist & 59,123 & 28 & 64,963 & 26 & 61,935 & 54 \\
\hline Subject Specialist & 75,055 & 22 & 70,698 & 12 & 73,518 & 34 \\
\hline \multicolumn{7}{|l|}{ Dept. Head: } \\
\hline Acquisitions & 63,699 & 26 & 57,183 & 8 & 62,166 & 34 \\
\hline Reference & 77,914 & 18 & 77,104 & 10 & 77,625 & 28 \\
\hline Cataloging & 73,337 & 24 & 64,112 & 4 & 72,020 & 28 \\
\hline Serials & $\neq$ & 5 & $\neq$ & 2 & 70,492 & 7 \\
\hline Documents/Maps & $\neq$ & 7 & $\neq$ & 2 & 71,695 & 9 \\
\hline Circulation & 61,620 & 24 & 58,514 & 5 & 61,084 & 29 \\
\hline Rare Books/Manuscripts & $\neq$ & 3 & $\neq$ & 1 & 75,779 & 4 \\
\hline Computer Systems & $\neq$ & 3 & $\neq$ & 5 & 77,600 & 8 \\
\hline Other & 73,100 & 23 & 72,652 & 12 & 72,946 & 35 \\
\hline \multicolumn{7}{|l|}{ Reference: } \\
\hline Over 14 years experience & 73,879 & 48 & 73,085 & 21 & 73,637 & 69 \\
\hline 10 to 14 years experience & 62,097 & 13 & 58,388 & 8 & 60,684 & 21 \\
\hline 5 to 9 years experience & 60,304 & 31 & 62,430 & 19 & 61,112 & 50 \\
\hline Under 5 years experience & 59,009 & 51 & 55,372 & 25 & 57,813 & 76 \\
\hline \multicolumn{7}{|l|}{ Cataloging: } \\
\hline Over 14 years experience & 62,094 & 20 & 69,828 & 5 & 63,641 & 25 \\
\hline 10 to 14 years experience & $\neq$ & 8 & $\neq$ & 1 & 55,997 & 9 \\
\hline 5 to 9 years experience & 57,120 & 10 & . & & 57,120 & 10 \\
\hline Under 5 years experience & $\neq$ & 4 & $\neq$ & 2 & 56,707 & 6 \\
\hline \multicolumn{7}{|l|}{ Other: } \\
\hline Over 14 years experience & 61,965 & 11 & 63,175 & 6 & 62,392 & 17 \\
\hline 10 to 14 years experience & $\neq$ & 6 & $\neq$ & 1 & 65,941 & 7 \\
\hline 5 to 9 years experience & $\neq$ & 3 & $\neq$ & 3 & 55,251 & 6 \\
\hline Under 5 years experience & $\neq$ & 9 & $\neq$ & 3 & 48,479 & 12 \\
\hline All Positions & $\$ 75,424$ & 494 & $\$ 82,097$ & 253 & $\$ 77,684$ & 747 \\
\hline
\end{tabular}

Canadian salaries are expressed in US dollars.

₹ Salary data are not published when fewer than four individuals are involved in either category.

. No positions reported in this category. 
Table 47: Number and Average Years of Experience of ARL University LaW Librarians by Position And SeX, FY 2009-2010

\begin{tabular}{|c|c|c|c|c|c|c|}
\hline \multirow[b]{2}{*}{ Position } & \multicolumn{2}{|c|}{ WOMEN } & \multicolumn{2}{|c|}{ MeN } & \multicolumn{2}{|c|}{ TOTAL } \\
\hline & Years & No. & Years & No. & Years & No. \\
\hline Associate Director & 25.6 & 34 & 19.4 & 24 & 23.0 & 58 \\
\hline Assistant Director & 21.5 & 24 & 23.3 & 10 & 22.0 & 34 \\
\hline Head, Law & 28.2 & 39 & 24.0 & 38 & 26.1 & 77 \\
\hline Functional Specialist & 11.4 & 28 & 9.8 & 26 & 10.6 & 54 \\
\hline Subject Specialist & 19.0 & 22 & 16.9 & 12 & 18.3 & 34 \\
\hline \multicolumn{7}{|l|}{ Dept. Head: } \\
\hline Acquisitions & 20.9 & 26 & 14.8 & 8 & 19.5 & 34 \\
\hline Reference & 14.9 & 18 & 19.8 & 10 & 16.6 & 28 \\
\hline Cataloging & 26.9 & 24 & 21.5 & 4 & 26.1 & 28 \\
\hline Serials & 23.0 & 5 & 7.0 & 2 & 18.4 & 7 \\
\hline Documents/Maps & 27.4 & 7 & 20.0 & 2 & 25.8 & 9 \\
\hline Circulation & 15.9 & 24 & 13.2 & 5 & 15.4 & 29 \\
\hline Rare Books/Manuscripts & 16.0 & 3 & 9.0 & 1 & 14.3 & 4 \\
\hline Computer Systems & 21.7 & 3 & 19.0 & 5 & 20.0 & 8 \\
\hline Other & 17.9 & 23 & 15.6 & 12 & 17.1 & 35 \\
\hline Public Services & 15.4 & 9 & 15.0 & 8 & 15.2 & 17 \\
\hline Technical Services & 10.8 & 14 & 14.0 & 2 & 11.2 & 16 \\
\hline Administrative Services & 19.5 & 6 & 14.3 & 3 & 17.8 & 9 \\
\hline Reference & 11.6 & 143 & 10.7 & 73 & 11.3 & 216 \\
\hline Cataloger & 17.4 & 42 & 18.8 & 8 & 17.6 & 50 \\
\hline All Positions & 17.6 & 494 & 15.8 & 253 & 17.0 & 747 \\
\hline
\end{tabular}


Table 48: Number and Average Salaries of ARL University Law Librarians by YeARS OF EXPERIENCE AND SeX, FY 2009-2010

\begin{tabular}{lccccccc}
\hline & \multicolumn{2}{c}{ WOMEN } & \multicolumn{2}{c}{ MEN } & \multicolumn{2}{c}{ TotaL } & \% OF \\
Experience & Salary & No. & Salary & No. & Salary & No. & TotaL \\
\hline $0-3$ years & $\$ 56,458$ & 70 & $\$ 54,409$ & 35 & $\$ 55,775$ & 105 & $14 \%$ \\
$4-7$ years & 60,703 & 66 & 60,500 & 36 & 60,631 & 102 & $14 \%$ \\
$8-11$ years & 68,700 & 44 & 70,355 & 37 & 69,456 & 81 & $11 \%$ \\
$12-15$ years & 69,566 & 64 & 87,351 & 26 & 74,704 & 90 & $12 \%$ \\
$16-19$ years & 72,751 & 42 & 80,604 & 22 & 75,451 & 64 & $9 \%$ \\
$20-23$ years & 84,884 & 41 & 83,140 & 27 & 84,192 & 68 & $9 \%$ \\
$24-27$ years & 81,625 & 38 & 104,873 & 21 & 89,900 & 59 & $8 \%$ \\
$28-31$ years & 85,595 & 49 & 110,547 & 25 & 94,024 & 74 & $10 \%$ \\
$32-35$ years & 99,619 & 44 & 108,617 & 21 & 102,526 & 65 & $9 \%$ \\
over 35 years & $\neq$ & 36 & $\neq$ & 3 & 106,669 & 39 & $5 \%$ \\
\hline All Positions & $\$ \mathbf{\$ 7 5 , 4 2 4}$ & $\mathbf{4 9 4}$ & $\mathbf{\$ 8 2 , 0 9 7}$ & $\mathbf{2 5 3}$ & $\mathbf{\$ 7 7 , 6 8 4}$ & $\mathbf{7 4 7}$ & $\mathbf{1 0 0 \%}$ \\
\hline
\end{tabular}

Canadian salaries are expressed in US dollars.

‡ Salary data are not published when fewer than four individuals are involved in either category. 

UNIVERSITY LiBRARY QUESTIONNAIRE AND INSTRUCTIONS 


\section{ARL Annual Salary Survey 2009-2010 \\ University Library Questionnaire \\ General And Data InPUt (Excel) Instructions}

\section{http://www.arl.org/stats/annualsurveys/salary/}

\section{General Overview}

- Use the newly available Web form for your data submission:

University Libraries: http://www.formspring.com/forms/?661042-c6BynijupA

Fill in Part I on the Web and upload your file for Part II through the same interface.

NOTE: You must complete the entire submission in a single session. The Web interface does NOT allow you to return and edit your information once it is submitted.

- This survey is concerned with professional positions only. Since the criteria for determining professional status vary among libraries, there is no attempt to define the term "professional." Each library should report the salaries of those staff members it considers professionals, irrespective of faculty status or membership in a collective bargaining unit, including, when appropriate, staff who are not librarians in the strict sense of the term, such as computer experts, systems analysts, budget officers, etc.

- Report individual salaries for the Main, Law, and Medical library on the separate template using Microsoft Excel (see http://www.arl.org/stats/annualsurveys/salary/salform09.shtml). A generic template is available. Add your institution's ARL Library Institution Code [LibID]. (See http://www.arl.org/stats/ annualsurveys/surveycoord/instno_inam.shtml if you do not know your code.)

- Use "Percent" to determine if an employee works full-time or part-time. All full-time employees have Percent $=1.00$, i.e., they work $100 \%$ of a full-time schedule. If Percent is less than 1.00 , then the employee works that fraction of a full-time schedule. For example, a $65 \%$ time appointment would be entered as 0.65 . Calculate the percent appointment by dividing the amount of time an employee works by the amount considered to be the norm for full-time employment at your institution. For example, if a full-time appointment at your institution is 12 months at 40 hours per week:

- A 9-month part-time appointment has Percent $=9 / 12$, or 0.75 .

- An appointment at 30 hours per week has Percent $=30 / 40$, also 0.75 .

$\circ \quad$ An appointment at 30 hours and 9 months has Percent $=0.75 \times 0.75=0.56$.

Enter Percent with two decimal points.

- Report salaries for both full-time and part-time professional positions. Salaries for part-time positions should NOT be converted to their full-time equivalents. Report the actual part-time salary paid and indicate the percent appointment for that employee in the appropriate column.

- Include salaries for all professional positions, regardless of whether the salaries come from regular library budget funds or from special funds such as research grants. Please include all professionals involved in the provision of library services, including contract-supported positions.

- The salary figures should be straight gross salary figures. Do not include fringe benefits. 
- Provide explanatory footnotes to the reported figures, when necessary, at the end of Part I. Footnotes will be included in the published survey, where appropriate.

- After all data have been entered, make a backup copy of the complete file for your institution's master file. Your backup should include individual names/ID numbers. NOTE: The data submitted to ARL should NOT include individual names/ID numbers, so ARL will NOT be able to supply a copy of your institution's complete file next year.

- Please return the questionnaire the ARL Statistics and Measurement Program by September 30, 2009. Be sure to keep a complete copy of your return, including the electronic version of the data for your files.

\section{INSTRUCTIONS}

\section{Part I: Summary Data (Microsoft Word Form)}

1. Part I of this survey deals with general information for the current fiscal year, 2009-2010.

2. Include the Beginning Professional Salary for Law and Medical libraries if included in the survey.

3. The Beginning Professional Salary is the salary that would be paid to a newly hired professional without experience, not necessarily the lowest professional salary paid. In reporting the beginning salary, please use a figure that is actually used or likely to be used for entry-level librarians hired by your library, even if it is your practice rarely to hire entry-level professionals without experience.

4. Please report the 2009-2010 Beginning Professional Salary to the best of your knowledge as it exists on July 1 , 2009. Do not delay returning your survey with the expectation that more information will be available later.

5. The 2009-2010 Average and Median Salary figures will be calculated by ARL from the individual data supplied.

6. Be sure to fill in the name of the reporting library and the name of the person who prepares the report.

\section{Part II: Individual Data (Microsoft Excel Form)}

1. Part II of this survey requests information on salary, sex, minority status, rank, and years of experience for all filled positions for fiscal year 2009-2010. The survey requests information for individuals; aggregate data for each institution will be generated by computer. Vacant positions should be excluded from your report.

2. Data for the Main, Law, and Medical libraries should be reported on separate Excel files.

3. Obtain the Excel file. These instructions assume that you have Microsoft Excel available for use. If not, or if you have trouble opening the files in Excel, please call the ARL Statistics and Measurement Program at (202) 296-2296 or e-mail stats@arl.org. 
4. The template Excel file is available at: http://www.arl.org/stats/annualsurveys/salary/salform09.shtml. This is a generic, blank file that can hold data for Main, Law, or Medical libraries. The file's name is "sal09xxxx. xls"; open the file and save it to your own computer by choosing "Save As" under the File menu. When saving the file, utilize ARL as the prefix for main library reports, use 09 to designate the year (2009-2010), and change the " $x x x x$ " in its name to your ARL institution code number, e.g., "ARL091150.xls." Note: use MED for medical libraries, e.g., "MED091150," and LAW to denote law libraries, e.g., "LAW091150.”

The file contains columns labeled as follows:

Required: Name/ID\#, LibID, Page, Line, Salary, Job, Sex, OEOcat, Yrsexp, Rank, Percent Optional: Hisp, NatAm, Asian, Black, HawPI, White

In the LibID column, enter your ARL Library Institution Code. (See http://www.arl.org/stats/ annualsurveys/surveycoord/instno_inam.shtml if you do not know your code.) If you leave this column blank we will fill it in for you when we receive the data.

Columns labeled "Page," "Line," and "Percent" are already filled for you. The numbers in the "Page" and "Line" columns will be used to identify these positions in case of data errors; do not change them. Ten "pages" of 25 lines each have been provided; if this is not sufficient to list all positions at your institution, copy and paste lines 1-25 of the last page as needed.

\section{Entering Data for Part II: Individual Data (Microsoft Excel Form)}

1. The "Name/ID\#" column is for your internal use, to enter and verify information for staff members by name. ARL does not require that you submit the information in this field to ARL. Please delete this column before sending the file to ARL. Upon receiving this file, ARL will delete any data in this column if you have not deleted them already.

2. The "LibID" will hold your institution's ARL number, for identification purposes. If you do not know your ARL number, you can find it on the Web under ARL Library Institution Codes. If you leave this column blank, it will be filled in by ARL staff.

3. "Salary" should be entered as it existed on July 1, 2009. Please do not hold up the reporting process for later salary adjustments. Include all filled positions and exclude all vacant positions. Report the actual salary paid. Do not adjust part-time salaries to their full-time equivalents; ARL will do this during the data analysis and verification stage. Do not include fringe benefits.

4. Each position can have only one "Job" code, to be taken from the following list:

DIRLIB Director of Libraries (includes Dean of Libraries and equivalent titles)

ASCDIR Associate Director

ASTDIR Assistant Director

HDMED Head, Medical Library (Human Medicine only)

HDLAW Head, Law Library

HDBR Head, Other Branch Library (including Veterinary Medicine)

FSPEC Functional Specialist

$\mathrm{ARCH} \quad$ Archivists/Curators

BUSI Budget/Fiscal/Business Manager/Facilities

HUMRES Human Resources/Training/Staff Development

ITS Information Technology Systems 


\section{ITW Information Technology Web Development}

ITP Information Technology Programming/Application Development

MEDIA Media/Multimedia Specialists (including graphics)

PRES Preservation/Conservation

SSPEC Subject Specialist

HDACQ Head, Acquisitions Department

HDCAT Head, Catalog Department/Unit

HDCIRC Head, Circulation

HDCOMP Head, Library and Computer Systems

HDDOC Head, Documents Department

HDMAP Head, Map Room/Department

HDRBM Head, Rare Book/Manuscripts Department

HDREF Head, Reference Department

HDSER Head, Serials Department

HDOTH Head, Other Department/Service/Agency

CAT

REF

PUBS

TECH

ADMIN

The position categories used in this survey are intended to correspond roughly with the activities carried on in libraries, not with any particular pattern of staff organization or nomenclature. Please use these categories in the manner you feel best applies to your library. If any individual has responsibilities described by more than one of the above categories, choose the category that is most typical of his/her general duties.

Associate or Assistant Director, and Head, Other Branch. Use these codes for all persons at these levels regardless of the area of specialty. If an assistant or associate director is also head of a department, choose the category that most reflects the general duties of the person currently in the position.

Specialists. These are of two kinds: Subject Specialists primarily build collections, but may also offer specialized reference and bibliographic services; Functional Specialists are media specialists or experts in management fields such as personnel, fiscal matters, systems, preservation, etc. Specialists may not be, strictly speaking, professional librarians (i.e., have an MLS). The "specialist" category would generally not be used for someone with significant supervisory responsibilities, who should instead be listed as a department head or assistant director (see also note under Assistant Department Head, below).

Functional Specialist sub-codes. Starting with the 2004-2005 Salary Survey, the ARL Statistics and Measurement Committee adopted a proposal from the ACRL Personnel Administrators and Staff Development Officers Discussion Group to break down the Functional Specialist category. For each position which would have been labeled FSPEC prior to 2004-05, instead please use one of the eight sub-codes (ARCH, BUSI, HUMRES, ITS, ITW, ITP, MEDIA, PRES) to describe that position. If you cannot determine which sub-code to use, please use the FSPEC code.

Department Heads. Department Heads not specifically included in the above list should be included under the category “Head, Other Department/Service/Agency." Head, Catalog Department should be used either for the department that handles all cataloging, or for the head of a specialized cataloging unit (e.g., copy cataloging or foreign languages). List the head of library automation and computer systems, applications, programming, etc. 
as HDCOMP unless that person is also an Associate or Assistant Director, in which case use the appropriate administrative code. If there is an intermediate level of management between an Associate or Assistant Director and the professionals who actually carry out the analysis, programming, etc., use HDCOMP to define that intermediate level. Professionals who carry out analysis, programming, etc., should be listed as functional specialists (FSPEC).

Head, Acquisitions Department. Use HDACQ for all of the following positions: (a) head of a department that is responsible for the selection of material (or management of selection activities carried out on a basis encompassing more than a single organizational unit), but not responsible for the placement of orders, payment of invoices, etc.; (b) head of a department responsible for the placement of orders, maintaining on-order files, payment of invoices, etc., but not responsible for selection decisions; (c) head of a department responsible for both the selection decisions (or coordination of selection activities) and for acquiring the material. Libraries that split these two functions between two departments should report more than one professional with the position HDACQ.

[Special note concerning Assistant Department Heads. Assistant Department Heads who are responsible for major units and spend the bulk of their time in supervision and revision of the work of others should also be listed as "Head, Other Department/Service/Agency." See additional subcodes below for Head, Cataloging, and Head, Other Department. However, Assistant Head positions responsible for small units or for supervision only in the absence of the head should be reported as non-supervisory or specialist positions as appropriate.]

Administrative. Please note that ADMIN is not only for Administrative Services and related positions, but also can be applied to Public Relations/Communications, Development/Fundraising, and all other administrative and/or professional positions which do not have a logical home elsewhere.

5. Please indicate "Sex" with the letter M or F, indicating male or female, respectively.

6. "OEOCat" minority status code, for US university libraries only, should be indicated with one of the following code numbers. (Leave blank if a Canadian library):

$$
\begin{aligned}
& 1=\text { Black } \\
& 2=\text { Hispanic } \\
& 3=\text { Asian or Pacific Islander } \\
& 4=\text { American Indian or Native Alaskan } \\
& 5=\text { Caucasian/Other }
\end{aligned}
$$

7. "YrsExp," or total years of professional experience. For most professional staff members this will mean counting the years since the MLS degree was awarded. When counting, do not subtract interim periods when an individual was not engaged in professional library employment if these periods are short in relation to the overall professional career. Count an academic year contract period as a full year. Be sure to include professional experience in previous positions and in other institutions. The figure should be rounded off to the nearest whole number; for example, a position with 14.5 years of experience would appear as 15 .

\section{Indicate "Rank" using the following system of codes:}

0 The library director. Some systems also use 0 for assistant and/or associate directors.

1 Lowest level in the rank structure, such as an entry-level position.

2-8 Successively higher levels; for example, 5 indicates a higher rank than 2.

9 Rank cannot be determined, or, the individual is outside the organization's rank structure. 
Responses concerning rank should be limited to professional librarians, and other professionals who occupy the same ranks as librarians. Leave the rank column blank for professionals who do not occupy these ranks or if the column is not applicable. For example, if the Library Business Officer holds a rank typically used for university administrators but not for librarians, do not supply a rank code for that individual, even if you have included salary and other data.

If multiple ranking structures are used for librarians and these structures are substantially different and not equivalent, enter individual rank information only for that group which represents the largest fraction of "rankand-file" librarians.

The maximum number of ranks reported here should not exceed the maximum number of rank-levels reported in Part I for individual data under Rank structure. When counting the total number of rank levels, include ranks that may be unoccupied at the present time due to circumstances like unusually high turnover, hiring freezes, etc.

9. "Percent" is used to determine if an employee works full-time or part-time. All full-time employees have Percent $=1.00$, i.e., they work $100 \%$ of a full-time schedule. If Percent is less than 1.00, then the employee works that fraction of a full-time schedule. For example, a $65 \%$ time appointment would be entered as 0.65 . Calculate the percent appointment by dividing the amount of time an employee works by the amount considered to be the norm for full-time employment at your institution. For example, if a full-time appointment at your institution is 12 months at 40 hours per week:

○ A 9-month part-time appointment has Percent $=9 / 12$, or 0.75 .

$\circ$ An appointment at 30 hours per week has Percent $=30 / 40$, also 0.75 .

○ An appointment at 30 hours and 9 months has Percent $=0.75 \times 0.75=0.56$.

Enter Percent with two decimal points.

Optional Questions: The US Office of Management and Budget has revised the Standards for the Classification of Federal Data on Race and Ethnicity and according to the new standard there will be five minimum categories for data on race (American Indian or Alaska Native, Asian, Black or African American, Native Hawaiian or Other Pacific Islander, and White) and one category for data on ethnicity ("Hispanic or Latino"). Respondents will be able to report more than one race by choosing multiple responses to the race question. The purpose of the revised classification is to reflect the increasing diversity of the US population that has resulted primarily from growth in immigration and in interracial marriages. The new standards were used by the Bureau of the Census in the 2000 decennial census. ${ }^{1}$ In light of these developments, we are collecting the new classification on race and ethnicity in the ARL Annual Salary Survey on an optional basis.

Ethnicity should be indicated by coding 1 to indicate if the person is of Hispanic or Latino ethnicity, and coding 0 otherwise. The definition of Hispanic or Latino ethnicity is: A person of Cuban, Mexican, Puerto Rican, Cuban, South or Central American, or other Spanish culture or origin, regardless of race.

Race should be indicated for US university libraries only, by choosing one or more responses among the five racial categories provided here; $1=y e s$ and $0=$ no. You can select multiple racial categories for a person. The definitions of the five racial categories, listed with their respective column names, are:

American Indian or Alaska Native (NatAm): A person having origins in any of the original peoples of North and South America (including Central America) who maintains tribal affiliation or community attachment.

1 http://www.census.gov/population/www/socdemo/race/racefactcb.html 
Asian (Asian): A person having origins in any of the original peoples of the Far East, Southeast Asia, or the Indian subcontinent including, for example, Cambodia, China, India, Japan, Korea, Malaysia, Pakistan, the Philippine Islands, Thailand, and Vietnam.

Black or African American (Black): A person having origins in any of the black racial groups of Africa.

Native Hawaiian or Other Pacific Islander (HawPI): A person having origins in any of the original peoples of Hawaii, Guam, Samoa, or other Pacific Islands.

White (White): A person having origins in any of the original peoples of Europe, the Middle East, or North Africa.

\section{Submitting the Data for Part I and Part II on the Web}

ARL is using the online services of FormSpring to collect the data. As part of its privacy policy, FormSpring pledges not to sell any collected information to third parties. For the complete FormSpring privacy policy, visit http://www.formspring.com/privacy.html. ARL also accepts Part I and Part II of the salary survey by e-mail from those users who may be uncomfortable submitting the files in FormSpring:

- University Libraries: http://www.formspring.com/forms/?661042-c6BynijupA

Be sure to have the electronic copy of your completed salary survey Excel file handy as you will be submitting this file via the FormSpring Web form. In addition to the completed Excel file, be prepared to provide the following information as well:

- The name, title, e-mail and phone number of the person who prepared the Excel file. The name, title, e-mail and phone number of your institution's contact person for the salary survey (if different from the person who prepared the Excel file)

- Indicate whether you are submitting salary information for one or more of the following: Main, Law, or Medical library, and the beginning professional salary and rank structure for each.

For professional salary list the salary that would be paid to a newly hired professional without experience (even if local practice discourages hiring entry-level professionals without experience). Please report the 2009-2010 beginning professional salary to the best of your knowledge as it existed on July 1, 2009.

For rank structure, list the number of unique levels in your institution's rank structure. If you have no levels in your rank structure, use 1 . The number reported here should be equal to the highest number in the "Rank" column of your Excel file (i.e., the number of levels reported in your Excel file should equal the number of levels reported here).

- The names of the libraries that are included and excluded in your figures for the 'general libraries' (these can be main campus libraries or branch campus libraries), as well as any other explanatory information, should be indicated in a footnote. In your footnotes, report any information that would clarify the figures submitted: the inclusion and exclusion of branch campus libraries, a reporting date that is sooner/ later than July 1, 2009, etc. Please make an effort to word your footnotes in a manner consistent with notes appearing in the published report, so that ARL can interpret your footnotes correctly.

Please return the completed questionnaire to the 
ARL Statistics and Measurement Program by September 30, 2009.

For assistance, contact Martha Kyrillidou (martha@arl.org) or Les Bland (les@arl.org) or

David Green (david@arl.org) or Gary Roebuck (gary@arl.org).

Tel. 202-296-2296 or fax 202-872-0884.

\section{http://www.arl.org/stats/annualsurveys/salary/}




\section{ARL Annual Salary Survey 2009-2010 \\ UNIVERSITY LIBRARY QUESTIONNAIRE}

Note: This is a copy of the form that you will submit electronically at: http://www.formspring.com/forms/?661042-c6BynijupA

\section{Part I: Summary Data}

Reporting Institution Date Returned to ARL

Report Prepared by (name)

Title

Email address Phone number

Contact person (if different)

Title

E-mail address Phone number

(Note: ARL will calculate the 2009-2010 median and average professional salaries for your library from the individual data you supply in Part II (Excel form) of this questionnaire.)
1. Beginning Professional Salary
Main
Law
Medical

Beginning professional salary for 2009-2010

(Note: The Information shown below must be completed for all three branches (i.e. Main, Law and Health Science Libraries) in Part1of the online form).

\section{Rank Structure.}

Indicate the number of levels in your institution's rank structure for professional librarians. You should report here the maximum number of rank levels, reported in Part II for individual data, under the Rank column.

1 level (i.e., no differentiated levels)

2 levels

3 levels 
4 levels

5 levels

more than 5 levels (please specify the number of levels:

\section{FOOTNOTES}

3a. Please list which libraries are included in the data submitted for the "general" libraries. These can be main campus libraries or branch campus libraries.

3b. Please list which libraries are NOT included in the data submitted for the "general" libraries. These can be main campus libraries or branch campus libraries.

Please indicate any other explanatory information in footnotes. These additional footnotes, if necessary, should be placed in the space below or on attached pages.

Please submit the completed questionnaire to the web form at: http://www.formspring.com/forms/?661042-c6BynijupA by September 30, 2009.

For assistance, contact Martha Kyrillidou (martha@arl.org) or Les Bland (les@arl.org), or call 202-296-2296 

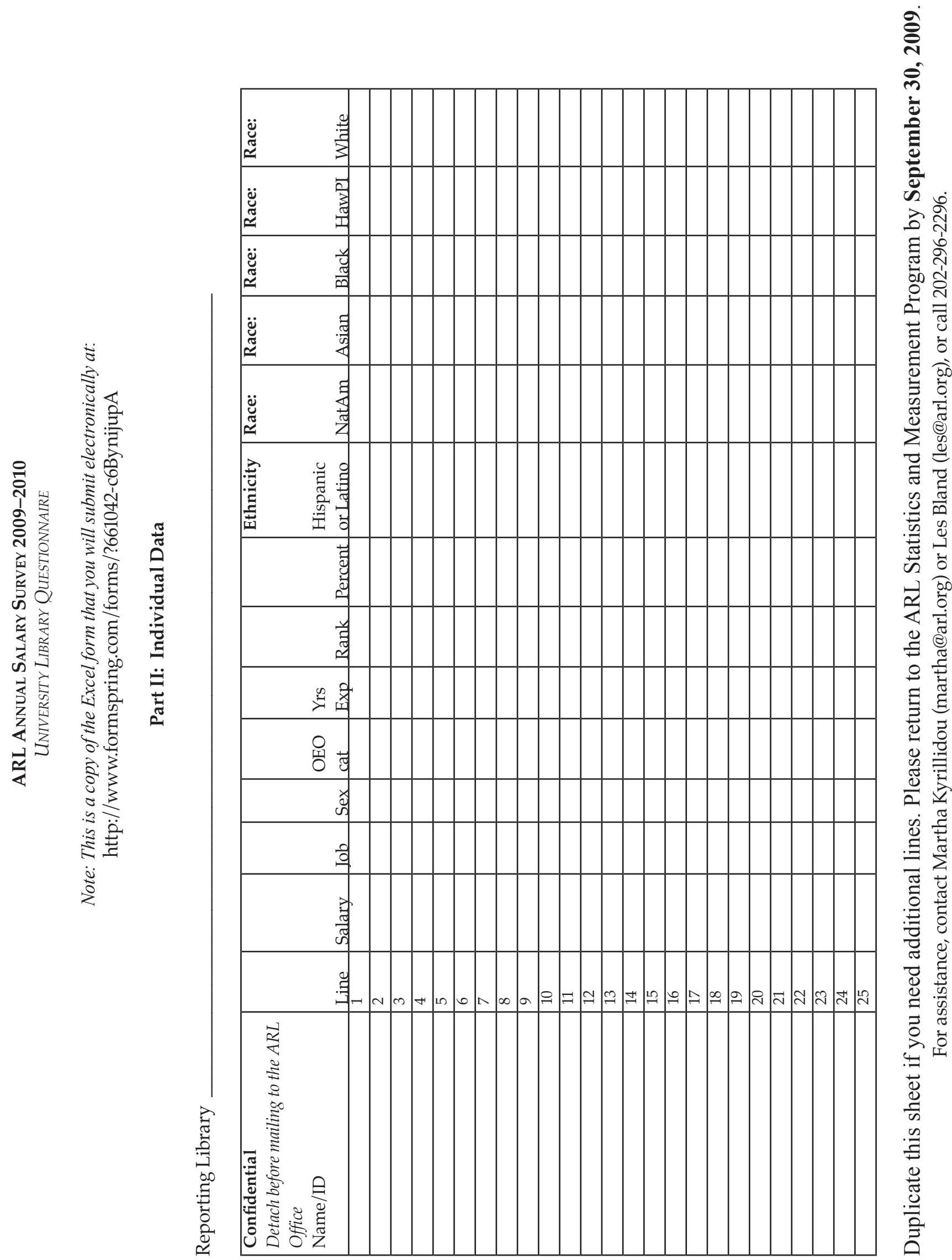
NonUniversity Library QUeSTIONNAIRE AND INSTRUCTIONS 


\title{
ARL Annual Salary Survey 2009-2010
}

\author{
NONUNIVERSITY LIBRARY QUESTIONNAIRE
}

\section{General Instructions for Completing the Questionnaire}

1. This survey is concerned with the salaries of professional positions only. Since the criteria for determining professional status vary among libraries, there is no attempt to define the term "professional." Each library should report the salaries of those staff members it considers professionals, irrespective of membership in a collective bargaining unit, and including, when appropriate, staff who are not librarians in the strict sense of the term, such as systems analysts, budget officers, etc.

2. Obtain the Word file. These instructions assume that you have Microsoft Word available for use. If not, or if you have trouble opening the files in Word, please call the ARL Statistics and Measurement Program at (202) 296-2296 or e-mail les@arl.org.

3. The template Word file is available at: http://www.arl.org/stats/annualsurveys/salary/salform09.shtml. This is a generic, blank form that can hold your data. The file's name is "sal09_nuform.doc"; open the file and save it to your own computer by choosing "Save As" under the File menu. When saving the file, utilize ARL as the prefix, use 09 to designate the year (2009-2010), and change the "xxxx" in its name to your ARL institution code number, e.g., "ARL099975.doc."

4. Salaries should be reported for all filled positions. Vacant positions should be excluded from your report.

5. Report 2009-2010 salaries as they exist on July 1, 2009. If the library normally increases salaries at a date after July 1 , and the salary as of that later date is known or can be estimated (within $\$ 100$ or so) by the time the questionnaire is due to be returned, please use the higher salary and footnote the effective date and/or whether the reported figures are known or estimated. Please do not hold up the reporting process for later salary adjustments.

6. The Median Salary is the salary that has an equal number of salaries above it and below it. In those libraries with an even number of positions, the median salary is the average of the two salaries that have an equal number of salaries above and below them.

7. The Beginning Professional Salary is the salary that would be paid to a professional without experience, not necessarily the lowest professional salary paid. In reporting the beginning salary, please use a figure that is actually used or likely to be used for entry-level librarians hired by your library.

8. Salaries should be reported for both full-time and part-time professional positions. However, salaries for part-time positions should be converted to their full-time equivalents before reporting; do not report the actual part-time salary paid.

9. Salaries should normally be reported on a 12-month basis. If an appointment is for 9 or 10 months at the option of the employee, the actual salary paid should be increased to its 12 -month equivalent. However, if appointments of less than 12 months are required by the employer, report the actual salary paid.

10. The salaries for all professional positions should be included, regardless of whether the salaries come from regular library budget funds or from special funds such as research grants. 
11. The salary figures should be straight gross salary figures. Do not include fringe benefits.

12. Explanatory footnotes to the reported figures may be provided when necessary. Footnotes will be included in the published survey.

13. Provide the name of the reporting library and the name of the person who prepares the report.

14. On the second page of the questionnaire (Part II) indicate the number of filled professional positions in each salary range for fiscal years 2008-2009 and 2009-2010.

15. Use the newly available Web form for your data submission:

(http://www.formspring.com/forms/?661053-c6BynijupA). Fill in Part I on the Web and upload your file for Part II through the same interface. NOTE: You must complete the entire submission in a single session. The Web interface does NOT allow you to return and edit your information once it is submitted.

Note: ARL is using the online services of FormSpring to collect the data. As part of its privacy policy, FormSpring pledges not to sell any collected information to third parties. For the complete FormSpring privacy policy, visit http://www.formspring.com/privacy.html. ARL also accepts Part I and Part II of the salary survey by e-mail attachment from those users who may be uncomfortable submitting the files in FormSpring.

\section{Please Submit the Web form by September 30, 2009.}

For assistance, contact Martha Kyrillidou (martha@arl.org) or Les Bland (les@arl.org) or David Green (david@arl.org) or Gary Roebuck (gary@arl.org). Tel. 202-296-2296 or fax 202-872-0884. 


\section{ARL Annual Salary Survey 2009-2010 \\ NONUNIVERSITY LIBRARY QUESTIONNAIRE}

Note: This is a copy of the form that you will submit electronically at:

http://www.formspring.com/forms/?661053-c6BynijupA

\section{Part I: Summary Data}

Reporting Institution Date Returned to ARL

Report Prepared by (name)

Title

Email address Phone number

Contact person (if different)

Title

Email address

Phone number

1. Complete the table on the back of this sheet by indicating the number of filled or temporarily vacant professional positions in each salary range for fiscal years 2007-2008 and 2008-2009.

2. Median professional salary for fiscal year 2009-2010:

3. Beginning professional salary for 2009-2010:

4. Footnotes (please compare with footnotes from surveys of previous years)

a. Law Library salaries are included.

Yes

No

We do not have a Law Library.

b. Medical Library salaries are included.

Yes

No

We do not have a Medical Library.

c. Branch libraries not included (please attach an additional sheet if necessary):

5. Other comments (please attach an additional sheet if necessary): 


\section{Part II Salaries:}

Indicate the number of filled professional positions in each salary range for fiscal years 2007-2008 and 2008-2009. Submit this form electronically (after Part 1) at: http://www.formspring.com/forms/?661053-c6BynijupA

\begin{tabular}{|c|c|c|}
\hline & \multicolumn{2}{|c|}{ Number of Positions } \\
\hline Salary Range & 2008-2009 & $2009-2010$ \\
\hline \multicolumn{3}{|l|}{ More than 250,000 } \\
\hline \multicolumn{3}{|l|}{$200,000-250,000$} \\
\hline \multicolumn{3}{|l|}{$175,000-199,999$} \\
\hline \multicolumn{3}{|l|}{$150,000-174,999$} \\
\hline \multicolumn{3}{|l|}{$140,000-149,999$} \\
\hline \multicolumn{3}{|l|}{$130,000-139,999$} \\
\hline \multicolumn{3}{|l|}{$120,000-129,999$} \\
\hline \multicolumn{3}{|l|}{$110,000-119,999$} \\
\hline \multicolumn{3}{|l|}{$100,000-109,999$} \\
\hline \multicolumn{3}{|l|}{$95,000-99,999$} \\
\hline \multicolumn{3}{|l|}{$90,000-94,999$} \\
\hline \multicolumn{3}{|l|}{$85,000-89,999$} \\
\hline \multicolumn{3}{|l|}{$80,000-84,999$} \\
\hline \multicolumn{3}{|l|}{$76,000-79,999$} \\
\hline \multicolumn{3}{|l|}{$74,000-75,999$} \\
\hline \multicolumn{3}{|l|}{$72,000-73,999$} \\
\hline \multicolumn{3}{|l|}{$70,000-71,999$} \\
\hline \multicolumn{3}{|l|}{$68,000-69,999$} \\
\hline \multicolumn{3}{|l|}{$66,000-67,999$} \\
\hline \multicolumn{3}{|l|}{$64,000-65,999$} \\
\hline \multicolumn{3}{|l|}{$62,000-63,999$} \\
\hline \multicolumn{3}{|l|}{$60,000-61,999$} \\
\hline \multicolumn{3}{|l|}{$58,000-59,999$} \\
\hline \multicolumn{3}{|l|}{$56,000-57,999$} \\
\hline \multicolumn{3}{|l|}{$54,000-55,999$} \\
\hline \multicolumn{3}{|l|}{$52,000-53,999$} \\
\hline $50,000-51,999$ & & \\
\hline $48,000-49,999$ & & \\
\hline $46,000-47,999$ & & \\
\hline $44,000-45,999$ & & \\
\hline $42,000-43,999$ & & \\
\hline $40,000-41,999$ & & \\
\hline $38,000-39,999$ & & \\
\hline $36,000-37,999$ & & \\
\hline $34,000-35,999$ & & \\
\hline $32,000-33,999$ & & \\
\hline $30,000-31,999$ & & \\
\hline less than 30,000 & & \\
\hline Total Number of Positions & & \\
\hline
\end{tabular}

Please submit the completed questionnaire to the Web form by September 30, 2008.

For assistance, contact Martha Kyrillidou (martha@arl.org) or Les Bland (les@arl.org) or David Green (david@arl.org) or Gary Roebuck (gary@arl.org). Tel. 202-296-2296 or fax 202-872-0884. 



\section{Footnotes to the ARL Annual Salary Survey, 2009-2010}

All data is as of 1 July 2009 unless otherwise noted.

\section{ALABAMA}

Libraries included: Amelia Gayle Gorgas Library, Angelo Bruno Business Library, McLure Education Library, Eric \& Sarah Rodgers Library for Science \& Engineering, and the W.S. Hoole Special Collections Library.

The listed Beginning Professional Salary (BPS) for the law library is with MLS \& JD.

\section{ALBERTA}

The following libraries are included: Bibliographic Services, HT Coutts Education Library, Humanities \& Social Sciences Library, Faculty Saint-Jean Library, Winspear Business Reference Library, Office of Staff Development and Training, Cameron Library (including Interlibrary Loans/Document Delivery, Financial Systems and Analysis, Science \& Technology Library, Information Technology Resource Services).

\section{ARIZONA}

The following libraries are included: Main Campus Library, Science-Engineering Library, Fine Arts Library, Special Collections Library, and The Center for Creative Photography.

Data for the medical library includes the Health Sciences Library in Phoenix.

\section{ARIZONA STATE}

University Libraries include ASU Tempe campus, ASU West campus, ASU Polytechnic campus, ASU Phoenix Downtown campus, and the Law Library.

Beginning Professional Salary for the Law Library is \$45,000 (entry level) and \$50,000 with JD.

\section{AUBURN}

Includes the Main campus library, Architecture Library, and the Vet Med Library.

\section{BOSTON}

The main library and law library have an 8-step rank structure.

\section{BOSTON COLLEGE}

Includes the Tip O’Neill Jr. Library, Bapst Art Library, John J. Burns Library of Rare Books and Special Collections, School of Social Work Library, Educational Resource Center, School of Theology and Ministry Library, and the Law Library.

\section{BRIGHAM YOUNG}

The Harold B. Lee Library (main campus library) and the Howard W. Hunter Law Library are included in this survey.

\section{BRITISH COLUMBIA}

The following libraries are included: Art+Architecture+Planning, Asian Library, David Lam Library, Education Library, Irving K. Barber Learning Centre, Koerner Library, Law Library, Library Processing Centre (Technical Services, IST), Life Science Libraries: Biomedical Branch Library, Hamber Library, St. Paul's Hospital Library, Woodward Biomedical Library, Music Library, Okanagan Library, Rare Books \& Special Collections, Robson Square, Science and Engineering, University Archives, and the Xwi7xwa Library (First Nations House of Learning).

Reading Rooms and Affiliated Libraries are not included.

The University of British Columbia Library system has no rank structure.

\section{BROWN}

Includes the John D. Rockefeller, Jr. Library, John Hay Library, Orwig Music Library, List Art Library, Sciences Library, and the John Carter Brown Library. 


\section{CALGARY}

Main Library submission includes: Mackimmie Library; Gallagher Library of Geology \& Geophysics; Business Library; Military Museum Library \& Archives. Health Libraries include: Health Sciences Library and Health Information Network Knowledge Centres. The Law Library is also included.

\section{CALIFORNIA, BERKELEY}

The following libraries are included: Doe, Moffitt, Bancroft, Anthropology, Art History/Classics, Astronomy- Mathematics- Statistics, Bioscience and Natural Resources, Business \& Economics, Chemistry, C.V. Starr East Asian Library (including Center for Chinese Studies), Earth Sciences, Education-Psychology, Engineering, Environmental Design, Music, Optometry, Physics, Public Health (including Health Sciences Information Services, and Occupational \& Environmental Health), Social Welfare libraries, and the Northern Regional Library Facility.

The following libraries are not included: Architectural (Slide Library), Continuing Education of the Bar, Earthquake Engineering, Ethnic Studies, Giannini, Institute of Governmental Studies, Institute of Industrial Relations, Institute of International Studies, and the Institute of Transport Library.

Beginning 2004-2005, UCB salary figures include administrative stipends, where applicable.

\section{CALIFORNIA, DAVIS}

Includes Peter J. Shields Library (Davis Campus); Physical Sciences \& Engineering Library (Davis Campus); Carlson Health Sciences Library (Davis Campus); Blaisdell Medical Center Library (Sacramento), and Agricultural \& Resource Economics Library (Davis).

Librarians who are department heads have received administrative stipends since July 1, 1999, but these stipends were not included until this report. We are including those stipends in the department head's salaries (as reported) and we plan to continue to do so in the future.

\section{CALIFORNIA, LOS ANGELES}

Includes the Arts Library, College Library (Undergraduate Library), Eugene and Maxine Rosenfeld Management Library, Music Library, Richard C. Rudolph East Asian Library, Science \& Engineering Library, Social Sciences and Humanities Library (Charles E. Young Research Library), and the Southern Regional Library Facility.

Includes data for 12 affiliated libraries on the UCLA campus including the 1) American Indian Studies Center, 2) Ralph M. Bunche African American Studies Center, 3) Asian American Studies Center, 4) Chicano Studies Research Center, 5) Ethnomusicology Archive, 6) Film \& Television Archive, 7) Graduate School of Education \& Information Studies, Department of Information Studies, 8) Institute for Social Science Research, 9) Latin American Center/Hispanic American Periodicals Index, 10) Olive View Medical Center, 11) Grace M. Hunt English Reading Room, and 12) William Andrews Clark Memorial Library.

Librarians who are department heads have received administrative stipends since January 1998; however, these stipends were not included in the salaries reported to ARL prior to 2003. Beginning with the 2003 survey, UCLA now includes those stipends in salaries reported for department heads. Interim department heads also receive stipends and these are reported in the survey.

The General Library Survey includes one Council of Library \& Information Resources (CLIR) Fellows.

\section{CALIFORNIA, RIVERSIDE}

Includes the following: Rivera Library (serving the College of Humanities, Arts and Social Sciences, School of Education, and the School of Business Administration), Science Library (serving the College of Natural \& Agricultural Sciences, the College of Engineering, and Biomedical Sciences), Palm Desert Campus Library (serving the Graduate School of Management \& the College of Humanities, Arts, and Social Sciences).

Media and Music Libraries are not included (there are no librarian employees in these facilities).

\section{CALIFORNIA, SAN DIEGO}

Includes the following: Arts Libraries, Social Sciences \& Humanities, International Relations \& Pacific Studies, Scripps Institution of Oceanography, Science \& Engineering, Special Collections Library, Biomedical Library, and the Medical Center Library.

\section{CALIFORNIA, SANTA BARBARA}

Includes the Main and Arts Libraries. 
The UCSB library has 8 levels in its rank structure.

\section{CASE WESTERN RESERVE}

Kelvin Smith Library, Kulas Music Library, Mandel School of Applied Social Sciences Library, and the Harris Library are included.

\section{CHICAGO}

All libraries, including law and medicine, are incorporated in this report.

\section{CINCINNATI}

Includes all main campus libraries.

Branch campus libraries are not included.

\section{CANADA INSTITUTE OF SCIENTIFIC AND TECHNICAL INFORMATION}

All branch libraries are included.

All values were reported in Canadian Dollars.

\section{COLORADO}

The following libraries are included: Norlin Library, Music Library, Earth Sciences Library, Business Library, Math/Physics Library, Engineering Library, and the Law Library.

\section{CONNECTICUT}

The Main, Law, Pharmacy, and Music \& Dramatic Arts libraries are included in this report.

The Beginning Professional Salary (BPS) for the Law Library has decreased because of the severe economic downturn.

\section{CORNELL}

Africana, Engineering, Entomology, Fine Arts, Geneva Experiment Station, Hotel Administration, Management, Mann Library, Math, Music, ILR, Olin/Kroch/Uris, Physical Sciences, Veterinary Medicine, Law, and Health Science libraries are included in this survey.

\section{DARTMOUTH}

Libraries included in this report: Baker-Berry Library, Feldberg Business \& Engineering Library, Kresge Physical Sciences Library, Paddock Music Library, Rauner Special Collections Library, Sherman Art Library Storage Library, Dana Biomedical Library, and the MatthewsFuller Health Sciences Library.

\section{DELAWARE}

Excludes the salary data of University of Delaware libraries not reporting directly to the Director of Libraries.

\section{DUKE}

The following libraries are included: Perkins/Bostock; Lilly, Music; Library Services Center; Divinity; Rare Book, Manuscript and Special Collections, University Archives, Law, and Health libraries.

The University did not authorize a merit pool for Fiscal Year 2010 (1 July 2009 - 30 June 2010). In lieu of annual merit increases in 2010, employees who have achieved a successful or exceptional rating on the 2008-2009 performance evaluation and who earn $\$ 50,000$ per year or less will receive a one-time $\$ 1,000$ payment (calculations based on a $1.0 \mathrm{FTE}$ ). There will be no merit increase or one time payment for University employees making more than $\$ 50,000$ per year. Librarians earning less than the $\$ 45 \mathrm{~K}$ minimum will be adjusted appropriately.

\section{EMORY}

The General Libraries, Theology Library, and Oxford College Library (Emory's two-year college) are included in this survey.

\section{FLORIDA}

Main libraries included Humanities and Social Sciences Library; Science Library; Journalism Library; Music Library; Architecture and Fine Arts Library; Education Library; Education Library; Latin America Collection; and the Maps Library.

Medical libraries data includes the main Health Science Center Library; a satellite branch in another city; and the Veterinary Medicine Reading Room. 
The Main and Medical libraries data is reported separately for this survey, but the systems were integrated in 2009, and now all libraries, with the exception of Law, report through the Dean of University Libraries.

For the purpose of this survey, Main and Medical libraries both have 7 rank levels.

\section{FLORIDA STATE}

The Allen Music Library, Goldstein College of Information Library are included with the main, law, and medical libraries in this report.

Panama City, Florida Branch, and the Sarasota Ringling branch libraries are not included.

We have a six-level rank system: 0- Dean; 1- Assistant Instructor; 2- Associate Instructor; 3- Instruction librarian; 4- Assistant Librarian; 5Associate Librarian; 6- University Librarian.

\section{GEORGETOWN}

Lauinger Library (Main), Woodstock Theological Library, Blommer Science Library, and the Bio-ethics Library are all included in this submission.

\section{GEORGIA}

Libraries included: Main Library, Science Library, Map Library, Student Learning Center Library, Curriculum Learning Center Library, several reading rooms, and experimental station libraries located throughout the State of Georgia.

The University of Georgia Law Library uses 9 levels in its rank structure.

\section{GUELPH}

The following main libraries are included: McLaughlin Library, and the OVC Learning Commons/Library. Branch Campus libraries included: Guelph Humber Learning Commons/Library and the Ridgetown Campus Library.

All salary values were reported in Canadian Dollars (\$CAD).

Individual rank data have been included only for professional librarians. Rank structure as follows: Library Director assigned rank $=0$; Assistant Librarian assigned rank $=1$; Associate Librarian assigned rank = 2; Librarian assigned rank $=3$; Non-librarian professionals assigned rank $=9$.

\section{HARVARD}

Includes the Law and Medical libraries as well as the Villa i Tatti and the Hellenic Center Libraries.

Excludes the Bilioteca Berenson (Florence, Italy) and the Dunbarton Oaks Research Libraries (Washington, DC).

Harvard uses a 9-step rank structure.

The salaries for Head of the Medical and Law libraries do not include compensation for the research and professorial duties of this position, yet they are considered full time.

\section{HAWAII}

Includes the Hamilton, Sinclair Libraries, and the Law library.

Both the Law Library and the Health Sciences Library are independent from the main university library and the directors report to their respective deans (Law and Medicine).

\section{HOUSTON}

The following are included: M.D. Anderson Library, Architecture and Art Library, Music Library, Weston A. Pettey Optometry Library, and the Pharmacy Library.

\section{HOWARD}

Founders Library and associated branches, including both the Law and the Louis Stokes Library (Health Sciences). 
The Moorland-Spingarn Research center is not included.

The Louis Stokes Health Sciences Library (LSHSL) is independent from the "general libraries" and is not a branch. However, we report these statistics with the general library.

\section{ILLINOIS, CHICAGO}

The medical libraries at Chicago, Peoria, Rockford, and Urbana are not included.

Illinois, Chicago has 6 levels for professional librarians: $6=$ Professor, $5=$ Associate Professor, $4=$ Assistant Professor, including visiting appointments, $3=$ Clinical Assistant Professor, 2=Instructor (Resident, Professional Library Associate), 1= Instructor (currently all 1 ranks are vacant).

\section{INDIANA}

Main campus libraries and the Indianapolis School of Law Library are included.

Not included: Dentistry and Medicine Library; IUPUI University Library; Herron School of Art Library, Columbus Library, and Science \& Engineering Library; and other campuses libraries at IU-East, IU-Kokomo, IU-Northwest, IU-Southeast, IU-South Bend, and IPFW-Fort Wayne.

\section{IOWA}

The following libraries are included: Main, Art, Biological Sciences, Engineering, Geoscience, Math, Music, Physics, and Psychology, Health Sciences, and Law.

All libraries mentioned above are on the same campus.

\section{IOWA STATE}

Includes Parks (main library) and the Veterinary Library.

\section{JOHNS HOPKINS}

Includes the Sheridan Libraries, Welch Medical Library, Friedheim Library, and the School of Advanced International Studies Library (SAIS).

\section{KANSAS}

Includes Regents Center Library, Edwards Campus (Overland Park, KS).

Excludes University of Kansas School of Medicine, Farha Library (Wichita, KS).

\section{KENT STATE}

Includes all libraries on the Kent State Kent campus: Main, Architecture, Chemistry/Physics, Fashion, Map, Music.

Includes libraries at Kent State branch campuses: Ashtabula, East Liverpool, Geauga, Salem, Stark, Trumbull, Tuscarawas.

The starting BPS we reported is for tenure-track faculty only. This figure is a significant increase over 2008-2009 and was determined by the university's collective bargaining agreement.

\section{KENTUCKY}

Includes the following: William T. Young Library (Main Campus Library), Agricultural Information Center, Design Library, Education Library, Engineering Library, Equine Library, Fine Arts Library, Science Library, and the Kentucky Transportation Center Library.

\section{LIBRARY OF CONGRESS}

Salaries include both professional and administrative positions.

\section{LOUISVILLE}

Main, Music, Art, University Archives, Law, and Kornhauser Health Sciences Library are included. 
MCMASTER

Includes the Innis Library, H.G. Thode Library and the Mills Memorial Library.

\section{MANITOBA}

Includes: William R. Newman Agriculture Library, Architecture/Fine Arts Library, Archives \& Special Collections, Elizabeth Dafoe Library, Father Harold Drake Library, St. John's College Library, Donald W. Craik Engineering Library, E.K. Williams Law Library, Albert D. Cohen Management Library, Eckhardt-Gramatte Music Library, Sciences and Technology Library, Neil John Maclean Health Sciences Library, Bill Larson Library, Carolyn Sifton-Helene Fuld Library, Concordia Hospital Library, J.W. Crane Memorial Library, Misercordia Health Centre Library, Riverview Health Centre Virtual Library, Seven Oaks General Hospital Library, and the Victoria General Hospital Library.

\section{MASSACHUSETTS}

The DuBois, Integrated Sciences \& Engineering, and Image Collection Libraries are included.

\section{MIAMI}

Rank structure for the main library is a 6-step structure (1-6).

The data submitted for Health Sciences are for the Calder and Psychiatry libraries.

\section{MICHIGAN}

Includes Hatcher Graduate; Shapiro Undergraduate; Shapiro Science; Art, Architecture \& Engineering; Askwith Media; Area Programs; Asia; Map; Special Collections; Spatial \& Numeric Data Services; Fine Arts; Social Work; Muesums; Music; Government Documents; Papyrology; Health Sciences Libraries; Law; Kresge Business Administration; Clements; Bentley and Gerald Ford Presidential Library.

Law Library beginning professional salary: \$48,000 for MILS degree only; \$60,000 for JD and MILS.

\section{MICHIGAN STATE}

Includes the Main, Biomedical, Physical Sciences, Math, Veterinary Medicine, Business, and Engineering libraries.

\section{MISSOURI}

Includes Main, Journalism, Engineering, Veterinary Medicine, and Archives Libraries.

\section{MIT}

MIT Libraries include: Barker Engineering Library, Science Library, Rotch Library for Architecture and Planning, Dewey Library for Management and Social Science, Humanities Library, Lewis Music Library (branch of Humanities), Rotch Visual Collections (branch of Rotch), and Institute Archives and Special Collections.

The number of levels in our rank structure is 9 . Rank level 1 is currently vacant.

\section{MONTREAL}

Libraries included: Environmental Development, Library and Information Sciences, Botany, Chemistry, Educational Resources, EducationCommunication-Psychology-Psychoeducation-Biology, Geography, Kinesiology, Humanities and Social Sciences, Rare Books and Special Collections, Mathematics and Computer Sciences, Veterinary, Music, Optometry, Physics, École polytechnique Library, Law, Health, and Paramedics.

\section{NATIONAL LIBRARY OF MEDICINE}

Data reported by the Federal fiscal year, which runs October 1 to September 30 of each calendar year.

\section{NEW MEXICO}

University of New Mexico Libraries include: Centennial Science \& Engineering Library, Fine Arts \& Design Library, Parish Memorial Library, Zimmerman Library, Law Library, and the Health Sciences Library.

Not included: Gallup Campus Branch Library, Los Alamos Campus Branch Library, Taos Campus Branch Library, Valencia Campus Branch Library. 
Definition of professional positions have been recalibrated to better represent professional activities and education. Public Service Librarian positions includes staff who function as "Ambassadors" and Teachers at a level above specialist.

\section{NEW YORK}

Includes: Elmer Holmes Bobst Library, Institute of Fine Arts, Courant Institute of Mathematical Sciences Library, Institute for Studies of the Ancient World, Real Estate Library, Law School Library, and the Medical School Library.

NYU's three ranks are indicated as follows: 1 - Library Associate; 2 - Assistant Curator; 3 - Associate Curator.

\section{NORTH CAROLINA STATE}

Includes: D.H. Hill (main) Library, Design Library, Natural Resources Library, Textiles Library, and the Veterinary Medicine Library.

\section{NORTHWESTERN}

Includes the Main Library, Science \& Engineering Library, Law Librar,y and Medical Library.

\section{NOTRE DAME}

Hesburgh Library (Main) includes: Architecture Library, Art Image Library, Business Information Center, Chemistry/Physics Library, Engineering Library, Kellogg/Kroc Information Center, and Mathematics Library. In addition, the Kresge Law Library has also been reported.

\section{OHIO}

Includes the main campus (Main Library plus Music/Dance Library) and five regional campus libraries (Eastern, Southern, Chillicothe, Lancaster, and Zanesville).

\section{OHIO STATE}

Includes all main campus libraries, regional campus libraries, and libraries of the Agricultural Technical Institute and the Ohio Agricultural Research and development center.

Several specialized department research libraries on the main campus which are not part of the University Libraries system are not included.

\section{OKLAHOMA STATE}

Includes the following main campus libraries: Edmon Low Library, Mary L. Williams Curriculum Materials Library, Cunningham Architecture Library, Brock Memorial Library at the Center for Veterinary Health Sciences, and the OSU Center for Health Sciences Library.

Includes the following branch campuses: OSU-Okmulgee Library, OSU-Oklahoma City Library, OSU-Tulsa Library.

An error was made during the processing of Oklahoma State's medical library for the 2008-2009 Salary Survey. An incorrect survey size of 8 "professionals" was reported—4 was the proper figure.

\section{OREGON}

Includes: Knight Library (main), Science Library, Architecture \& Allied Arts Library, Portland Library \& Learning Commons, and the Law Library.

Explanation of our rank system: 1 = Assistant Professor, 2 = Associate Professor, 3 = Professor, $9=$ Unranked, $0=$ Dean of Libraries.

\section{PENNSYLVANIA}

Includes: Museum, Fine Arts, Physical Sciences, Center for Advanced Judaic Studies, Rare Book \& Manuscript, Music, Business, and Law Libraries.

\section{PENNSYLVANIA STATE}

All the libraries at the University Park (Main Campus) are included. Libraries at the following branches: Abington, Altoona, Beaver, Berks, Brandywine, DuBois, Erie, Fayette, Great Valley, Greater Allegheny, Harrisburg, Hazleton, Lehigh Valley, Mont Alto, New Kensington, Shenango, Schuylkill, Wilkes-Barre, Worthington-Scranton, and York are also included. 
PITTSBURGH

Includes the University Library System and excludes branch campuses at Titusville, Johnstown, Bradford, and Greensburg.

\section{PRINCETON}

Includes the Firestone Library, Marquand Library of Art \& Archaeology, Mendel Music Library, Architecture Library, Stokes Library, East Asian Library, Engineering Library, Princeton Plasma Physics Library, Lewis Library, Psychology Library, and the Cotsen Children's Library.

The Lewis Library has incorporated the following branch libraries: Science and Technology, Biology and Life Sciences, Chemistry, Astrophysics, Physics, Math, and Geographical Sciences.

The Beginning Professional Salary (BPS) has increased from that paid in 2008-2009.

\section{PURDUE}

Includes the library system on the West Lafayette campus, consisting of 11 subject libraries, an undergraduate library, and an archives and special collections unit.

Excludes libraries at the regional campuses: Purdue North Central (Westville), Purdue Calumet (Hammond), and Indiana UniversityPurdue University, Fort Wayne.

\section{RICE}

In this reporting period, Rice University provided raises only to those making less than $\$ 60,000$ yearly.

\section{ROCHESTER}

Includes River Campus Libraries, Edward G. Miner Medical Library, and the Sibley Music Library.

\section{RUTGERS}

Includes the Newark Law Campus, Camden Law Campus, Research and Instructional Services, John Cotton Dana Library, Paul Robeson Library, Technical and Automated Services, Alexander Library, Mabel Smith Douglas Library, Kilmer Library, and the Library of Science and Medicine (and branches).

Excludes the School for Management and Labor Relations, and the Center for Alcohol Studies.

Salaries are unchanged from last year (2008-2009) because negotiated raises have been delayed (Newark Law Library).

\section{SASKATCHEWAN}

Includes Main Library, Education Library, and the Natural Sciences, Law, Medical, VetMed, and Engineering libraries.

Numbers of employees based upon those working at the library as of 1 July 2009.

\section{SOUTH CAROLINA}

Includes the Thomas Cooper Library, South Caroliniana Library, Moving Image Research Collection, South Carolina Political Collections, Music Library, Math Library, Business Library, Law Library, and Medical Library.

\section{SOUTHERN CALIFORNIA}

Includes all main campus libraries as well as the Norris Medical Library.

Beginning professional salary remains the same as 2008-2009.

\section{SUNY-ALBANY}

Libraries included are University Library, Science Library, and Dewey Graduate Library.

Salaries include 3\% union-negotiated increase, administrative stipends and SL-2 ranks. Vacant positions are not included. We have included our SL-2 ranks in this report so our survey size has increased from 2008-2009. 


\section{SUNY-BUFFALO}

Includes the Arts and Sciences Libraries, Music Library, Special Collections (Archives, Poetry, and Rare Books).

Excludes temporary hires, employees at the SL-2 salary level, and classified staff.

\section{SYRACUSE}

Includes the Main campus library, Science and Technology library, Geology Library, Law Library, and the Math Library.

\section{TEMPLE}

Includes: Paley Library; Science, Engineering \& Architecture Library; Charles L. Blockson Afro-American Collection; Ambler Campus Library.

Main Library minimum beginning professional salary of $\$ 44,044$ is based on an 11 -month contract. The minimum for a 10 -month contract is $\$ 40,150$.

\section{TENNESSEE}

Main (University of Tennessee) Library, Memphis Health Sciences Library, and Knoxville Hospital Preston Medical Library are included.

The Memphis Health Sciences Library has a 4-rank salary structure and the Knoxville Hospital has a 3-rank structure.

Beginning salary for Memphis Hospital is $\$ 45,000$ and $\$ 38,000$ for Knoxville Hospital.

\section{TEXAS}

Figures are as of August 31, 2009. Included are the Dolph Briscoe Center for American History, Tarlton Law Library, the Harry Ransom Humanities Research Center, and the University of Texas Libraries.

\section{TEXAS A\&M}

The following libraries are included: Sterling C. Evans Library, Library Annex, Cushing Memorial Library, Technical Reference Center (Architecture Library), Medical Sciences Library, West Campus Library, Policy Sciences and Economics Library, Jack K. Williams Library (Galveston), and the Texas A\&M University Library at Qatar.

The 1's (Lecturers) and 2's (Senior Lecturers) in our rank structure are non-tenured track appointments with a different salary schedule than our tenure track employees (3 - Assistant Professor, 4 - Associate Professor, 5 - Professor). 9's are Library professionals that work in specialized areas and have a different salary structure than either the tenured or non-tenured appointments.

\section{TEXAS TECH}

The Law Library reports 4 levels in their rank structure and the Health Sciences Library reports 5 levels in their rank structure.

Figures reported are as of 09/01/2009.

\section{TORONTO}

The proposed July 01, 2009 across-the-board pay increase has not yet been finalized.

Salary increases reflect movement within the rank structure (e.g., promotions).

\section{UTAH}

Includes the Main Library, Eccles Health Sciences Library, and the Quinney Law Library.

The Spencer S. Eccles Library is the main medical library. It is not a branch library reporting to Marriott Library. The medical library reports directly to the VP of Health at the University of Utah.

\section{VANDERBILT}

The data submitted includes the Central Library, Divinity Library, Peabody Library, Management Library, Science \& Engineering Library, Special Collections and University Archives, centralized Technical Services, Library Information Technology Services, Law Library, Health Sciences Library, Library Administration, and the Television News Archive. 


\section{VIRGINIA}

Includes the following University of Virginia Libraries: Alderman (Main), Astronomy, Biology/Psychology, Chemistry, Clemons (Undergraduate), Education, Fiske Kimball Fine Arts, Math, Music, Physics, Brown Science/Engineering, Small Special Collections, Darden

Graduate Business, Claude Moore Health Sciences, and the Authur Morris Law Library.

Excludes the John Cook Wyllie Library at the University of Virginia College at Wise.

\section{WASHINGTON}

Includes librarians on the Seattle, Bothell, and Tacoma campuses of the University of Washington.

\section{WASHINGTON STATE}

Includes all main campus libraries and the following branches: WSU-Riverpoint Campus, WSU-Tri-Cities, WSU-Vancouver, and the WSUEnergy Library.

\section{WASHINGTON UNIVERSITY-ST. LOUIS}

Includes the Central Library and departmental libraries in Art \& Architecture, Biology, Business, Chemistry, Earth \& Planetary Sciences, East Asian, Music, Physics, Social Work, and West Campus Library. The Law and Medical libraries are also included.

\section{WATERLOO}

Includes the Dana Porter; Davis Centre; University Map Library, and Musagetes Architecture Libraries.

We have a 6-level rank structure.

\section{WAYNE STATE}

The "general libraries" include the Purdy/Kresge Library, Science and Engineering Library, and the Undergraduate Library.

The libraries not included in the "general libraries" are the Neef Law Library and the Shiffman Medical Library, which are reported separately, and the Reuther Archives of Labor and Urban Affairs, which is not included in this report.

The decrease in the number of professionals reported (decreased from 2008-2009) is not the result of lost positions. It stems from our having looked more closely at which staff members were classified as professionals. The examination revealed that a certain level of staff that we have consistently counted as support staff when responding to the ARL Annual Survey were being counted as professional staff when responding to the Salary Survey. In order to be consistent and to accurately portray our libraries, we have now corrected this.

\section{WESTERN ONTARIO}

The D.B. Weldon Library, Business Library, Education Library, Music Library, Allyn \& Betty Taylor Library are included in this survey.

Affiliated College Libraries are not included. These are King's University College Library, Huron University College Library, and the Brescia University College Library.

July 1, 2009 salary increases are currently under negotiation.

\section{WISCONSIN}

Includes General Library System (Memorial, Steenbock, College, Art, Music, Special Collections, Geology, Geography, Biology, Physics, Social Work, Business, Chemistry, Math, Social Science Reading Room), Law, Medical, and the Wendt Engineering Library.

Our rank structure is as follows: 1 = Associate Academic Librarian, 2 = Academic Librarian, 3 = Senior Academic Librarian, $4=$ Distinguished Academic Librarian, 5 = Assistant/Associate Director, 6 = Director of the Steenbock, College, Memorial, Wendt, Law or Medical Libraries, 7 = Deputy Director General Library System, 0 = Director General Library. 
YALE

Includes: the African Collection, Anthropology Library, Area Studies Library, Arts Library, Arts of the Book Collection, Astronomy Library, Babylonian Collection, Bass Library, Beinecke Rare Book and Manuscript Library, Center for British Art, Chemistry Library, Classics Library, Divinity School Library, Drama Library, East Asia Library, Economic Growth Center Collection, Engineering and Applied Science Library, Epidemiology and Public Health Library, Film Study Center, Forestry and Environmental Studies Library, Fortunoff Video Archive for Holocaust Testimonies, Geographic Information Systems (GIS) at Yale, Geology Library, Government Documents and Information Center, Historical Sound Recordings, Judaica Collection, Kline Science Library, Latin American Collection, Lewis Walpole Library, Library Shelving Facility, Manuscripts and Archives at SML, Map Collection, Mathematics Library, Medical Historical Library, Microform Reading Room, Mudd Library, Music Library, Near Eastern Collection, Newspaper Reading Room, Nursing Library and Information Resources, Ornithology Library, Slavic and East European Collection, Social Science Libraries and Information Services, Southeast Asia Collection, Special Collections at Yale, Sterling Memorial Library, Visual Resources Collection, Yale Center for British Art, Medical Library, and the Law Library.

\section{YORK}

Please note that a new faculty collective agreement has been negotiated and beginning salary for 2009-2010 remains at the same level as 2008-2009 until details are finalized. 


\section{ARL Member Libraries as of January 1, 2010}

The Association of Research Libraries (ARL) represents the interests of 124 libraries that serve major North American research institutions. ARL operates as a forum for the exchange of ideas and as an agent for collective action to influence the forces affecting the ability of these libraries to meet the future needs of scholarship. The ARL Statistics and Measurement program is organized around identifying, collecting, analyzing, and distributing quantifiable information describing the characteristics of research libraries. The program offers publications and special member services, and collaborates with other national and international library statistics programs.

\begin{tabular}{|c|c|c|c|}
\hline Institution & Category & Full Name of Institution & Location \\
\hline Alabama & $S$ & University of Alabama & Tuscaloosa, Alabama \\
\hline Alberta & $\mathrm{C}$ & University of Alberta & Edmonton, Alberta \\
\hline Arizona & $S$ & University of Arizona & Tucson, Arizona \\
\hline Arizona State & $S$ & Arizona State University & Tempe, Arizona \\
\hline Auburn & $S$ & Auburn University & Auburn, Alabama \\
\hline Boston & $\mathrm{P}$ & Boston University & Boston, Massachusetts \\
\hline Boston College & $\mathrm{P}$ & Boston College & Boston, Massachusetts \\
\hline Brigham Young & $\mathrm{P}$ & Brigham Young University & Provo, Utah \\
\hline British Columbia & $\mathrm{C}$ & University of British Columbia & Vancouver, British Columbia \\
\hline Brown & $\mathrm{P}$ & Brown University & Providence, Rhode Island \\
\hline Berkeley, California & $S$ & University of California, Berkeley & California, Berkeley \\
\hline Calgary & $\mathrm{C}$ & University of Calgary & Calgary, Alberta \\
\hline California, Davis & $S$ & University of California, Davis & Davis, California \\
\hline California, Irvine & $\mathrm{S}$ & University of California, Irvine & Irvine, California \\
\hline California, Los Angeles & $S$ & University of California, Los Angeles & Los Angeles, California \\
\hline California, Riverside & $S$ & University of California, Riverside & Riverside, California \\
\hline California, San Diego & $S$ & University of California, San Diego & La Jolla, California \\
\hline California, Santa Barbara & $\mathrm{S}$ & University of California, Santa Barbara & Santa Barbara, California \\
\hline Case Western Reserve & $\mathrm{P}$ & Case Western Reserve University & Cleveland, Ohio \\
\hline Chicago & $\mathrm{P}$ & University of Chicago & Chicago, Illinois \\
\hline Cincinnati & S & University of Cincinnati & Cincinnati, Ohio \\
\hline Colorado & $\mathrm{S}$ & University of Colorado & Boulder, Colorado \\
\hline Colorado State & S & Colorado State University & Fort Collins, Colorado \\
\hline Columbia & $\mathrm{P}$ & Columbia University & New York, New York \\
\hline Connecticut & S & University of Connecticut & Storrs, Connecticut \\
\hline Cornell & $\mathrm{P}$ & Cornell University & Ithaca, New York \\
\hline Dartmouth & $\mathrm{P}$ & Dartmouth College & Hanover, New Hampshire \\
\hline Delaware & $S$ & University of Delaware & Newark, Delaware \\
\hline Duke & $\mathrm{P}$ & Duke University & Durham, North Carolina \\
\hline Emory & $\mathrm{P}$ & Emory University & Atlanta, Georgia \\
\hline Florida & S & University of Florida & Gainesville, Florida \\
\hline Florida State & S & Florida State University & Tallahassee, Florida \\
\hline George Washington & $\mathrm{P}$ & George Washington University & Washington, DC \\
\hline Georgetown & $\mathrm{P}$ & Georgetown University & Washington, DC \\
\hline
\end{tabular}




\begin{tabular}{|c|c|c|c|}
\hline Institution & Category & Full Name of Institution & Location \\
\hline Georgia & $S$ & University of Georgia & Athens, Georgia \\
\hline Georgia Tech & $S$ & Georgia Institute of Technology & Atlanta, Georgia \\
\hline Guelph & $\mathrm{C}$ & University of Guelph & Guelph, Ontario \\
\hline Harvard & $\mathrm{P}$ & Harvard University & Cambridge, Massachusetts \\
\hline Hawaii & $S$ & University of Hawaii & Honolulu, Hawaii \\
\hline Houston & $S$ & University of Houston & Houston, Texas \\
\hline Howard & $\mathrm{P}$ & Howard University & Washington, DC \\
\hline Illinois, Chicago & $\mathrm{S}$ & University of Illinois at Chicago & Chicago, Illinois \\
\hline Illinois, Urbana & $S$ & University of Illinois at Urbana & Urbana, Illinois \\
\hline Indiana & $S$ & Indiana University & Bloomington, Indiana \\
\hline Iowa & $S$ & University of Iowa & Iowa City, Iowa \\
\hline Iowa State & $S$ & Iowa State University & Ames, Iowa \\
\hline Johns Hopkins & $\mathrm{P}$ & Johns Hopkins University & Baltimore, Maryland \\
\hline Kansas & $\mathrm{S}$ & University of Kansas & Lawrence, Kansas \\
\hline Kent State & $\mathrm{S}$ & Kent State University & Kent, Ohio \\
\hline Kentucky & $\mathrm{S}$ & University of Kentucky & Lexington, Kentucky \\
\hline Laval & $\mathrm{C}$ & Laval University & Quebec, Quebec \\
\hline Louisiana State & $\mathrm{S}$ & Louisiana State University & Baton Rouge, Louisiana \\
\hline Louisville & S & University of Louisville & Louisville, Kentucky \\
\hline McGill & $\mathrm{C}$ & McGill University & Montreal, Quebec \\
\hline McMaster & $\mathrm{C}$ & McMaster University & Hamilton, Ontario \\
\hline Manitoba & $\mathrm{C}$ & University of Manitoba & Winnipeg, Manitoba \\
\hline Maryland & $\mathrm{S}$ & University of Maryland & College Park, Maryland \\
\hline Massachusetts & $\mathrm{S}$ & University of Massachusetts & Amherst, Massachusetts \\
\hline MIT & $\mathrm{P}$ & Massachusetts Institute of Technology & Cambridge, Massachusetts \\
\hline Miami & $\mathrm{P}$ & University of Miami & Coral Gables, Florida \\
\hline Michigan & $\mathrm{S}$ & University of Michigan & Ann Arbor, Michigan \\
\hline Michigan State & $\mathrm{S}$ & Michigan State University & East Lansing, Michigan \\
\hline Minnesota & $\mathrm{S}$ & University of Minnesota & Minneapolis, Minnesota \\
\hline Missouri & $\mathrm{S}$ & University of Missouri & Columbia, Missouri \\
\hline Montreal & $\mathrm{C}$ & University of Montreal & Montreal, Quebec \\
\hline Nebraska & S & University of Nebraska-Lincoln & Lincoln, Nebraska \\
\hline New Mexico & S & University of New Mexico & Albuquerque, New Mexico \\
\hline New York & $\mathrm{P}$ & New York University & New York, New York \\
\hline North Carolina & $\mathrm{S}$ & University of North Carolina & Chapel Hill, North Carolina \\
\hline North Carolina State & S & North Carolina State University & Raleigh, North Carolina \\
\hline Northwestern & $\mathrm{P}$ & Northwestern University & Evanston, Illinois \\
\hline Notre Dame & $\mathrm{P}$ & University of Notre Dame & Notre Dame, Indiana \\
\hline Ohio & $S$ & Ohio University & Athens, Ohio \\
\hline Ohio State & $S$ & Ohio State University & Columbus, Ohio \\
\hline Oklahoma & $S$ & University of Oklahoma & Norman, Oklahoma \\
\hline Oklahoma State & S & Oklahoma State University & Stillwater, Oklahoma \\
\hline
\end{tabular}




\begin{tabular}{|c|c|c|c|}
\hline Institution & Category & Full Name of Institution & Location \\
\hline Oregon & S & University of Oregon & Eugene, Oregon \\
\hline Pennsylvania & $\mathrm{P}$ & University of Pennsylvania & Philadelphia, Pennsylvania \\
\hline Pennsylvania State & S & Pennsylvania State University & University Park, Pennsylvania \\
\hline Pittsburgh & S & University of Pittsburgh & Pittsburgh, Pennsylvania \\
\hline Princeton & $\mathrm{P}$ & Princeton University & Princeton, New Jersey \\
\hline Purdue & S & Purdue University & West Lafayette, Indiana \\
\hline Queen's & $\mathrm{C}$ & Queen's University & Kingston, Ontario \\
\hline Rice & $\mathrm{P}$ & Rice University & Houston, Texas \\
\hline Rochester & $\mathrm{P}$ & University of Rochester & Rochester, New York \\
\hline Rutgers & S & Rutgers University & New Brunswick, New Jersey \\
\hline Saskatchewan & $\mathrm{C}$ & University of Saskatchewan & Saskatoon, Saskatchewan \\
\hline South Carolina & S & University of South Carolina & Columbia, South Carolina \\
\hline Southern California & $\mathrm{P}$ & University of Southern California & Los Angeles, California \\
\hline Southern Illinois & S & Southern Illinois University & Carbondale, Illinois \\
\hline SUNY-Albany & S & University at Albany, State University of New York & Albany, New York \\
\hline SUNY-Buffalo & S & University at Buffalo, State University of New York & Buffalo, New York \\
\hline SUNY-Stony Brook & S & State University of New York at Stony Brook & Stony Brook, New York \\
\hline Syracuse & $\mathrm{P}$ & Syracuse University & Syracuse, New York \\
\hline Temple & S & Temple University & Philadelphia, Pennsylvania \\
\hline Tennessee & S & University of Tennessee & Knoxville, Tennessee \\
\hline Texas & S & University of Texas & Austin, Texas \\
\hline Texas A\&M & S & Texas A\&M University & College Station, Texas \\
\hline Texas Tech & S & Texas Tech University & Lubbock, Texas \\
\hline Toronto & $\mathrm{C}$ & University of Toronto & Toronto, Ontario \\
\hline Tulane & $\mathrm{P}$ & Tulane University & New Orleans, Louisiana \\
\hline Utah & S & University of Utah & Salt Lake City, Utah \\
\hline Vanderbilt & $\mathrm{P}$ & Vanderbilt University & Nashville, Tennessee \\
\hline Virginia & S & University of Virginia & Charlottesville, Virginia \\
\hline Virginia Tech & S & Virginia Polytechnic Institute \& State University & Blacksburg, Virginia \\
\hline Washington & $S$ & University of Washington & Seattle, Washington \\
\hline Washington State & S & Washington State University & Pullman, Washington \\
\hline Washington U.-St. Louis & $\mathrm{P}$ & Washington University & St. Louis, Missouri \\
\hline Waterloo & $\mathrm{C}$ & University of Waterloo & Waterloo, Ontario \\
\hline Wayne State & S & Wayne State University & Detroit, Michigan \\
\hline Western Ontario & $\mathrm{C}$ & University of Western Ontario & London, Ontario \\
\hline Wisconsin & S & University of Wisconsin & Madison, Wisconsin \\
\hline Yale & $\mathrm{P}$ & Yale University & New Haven, Connecticut \\
\hline York & $\mathrm{C}$ & York University & North York, Ontario \\
\hline Boston Public Library & $\mathrm{N}$ & Boston Public Library & Boston, Massachusetts \\
\hline Canada Inst. SciTech Info. & $\mathrm{X}$ & Canada Inst. for Scientific \& Technical Information & Ottawa, Ontario \\
\hline Center for Research Libs. & $\mathrm{N}$ & Center for Research Libraries & Chicago, Illinois \\
\hline
\end{tabular}




Library of Congress
Natl. Agricultural Lib.
Lib. \& Archives Canada
Natl. Library of Medicine
New York Public Library
New York State Library
Smithsonian Institution
S - U.S. public university
P - U.S. private university
C - Canadian university
N - U.S. nonuniversity
X-Canadian nonuniversity

Library of Congress

National Agricultural Library

Library and Archives Canada

National Library of Medicine

New York Public Library

New York State Library

Smithsonian Institution
Washington, DC

Beltsville, Maryland

Ottawa, Ontario

Bethesda, Maryland

New York, New York

Albany, New York

Washington, DC 
From 1998-1999 to 1999-2000 a number of new tables were added to ARL Annual Salary Survey publication and some old tables were renumbered. As a result of these changes, a new section was added, entitled US ARL University Libraries, which includes Tables 26 and 29. Two new tables showing averages for all US and Canadian institutions were also included as Table 3 and Table 4 . The table below maps the old table-numbering scheme to the new one for purposes of comparison.

\section{Old New}

\section{SALARY LeVELS FOR STAFF IN ARL LibraRies}

$1 \quad 1 \quad$ Distribution by Salary Level

$12 \quad 2$ Salary Trends in ARL University Libraries

N/A 3 Salary Trends in US ARL University Libraries

N/A $\quad 4 \quad$ Salary Trends in Canadian ARL University Libraries

\section{ARL NONUNIVERSITY LIBRARIES}

25 Median and Beginning Professional Salaries in ARL Nonuniversity Libraries

$3 \quad 6 \quad$ Salary Trends in ARL Nonuniversity Libraries

\section{ARL UNIVERSITY LIBRARIES}

47 Filled Positions; Average, Median, Beginning Professional Salaries, And Average Years of Professional Experience in ARL University Libraries, FY 1999-2000

$5 \quad 8$

8 Beginning Professional Salaries in ARL University Libraries; Rank Order Table, FY 1998-1999

69

Beginning Professional Salaries in ARL University Libraries; Rank Order Table, FY 1999-2000

Median Professional Salaries in ARL University Libraries; Rank Order Table, FY 1998-1999

Median Professional Salaries in ARL University Libraries; Rank Order Table, FY 1999-2000

Average Professional Salaries in ARL University Libraries; Rank Order Table, FY 1998-1999

Average Professional Salaries in ARL University Libraries; Rank Order Table, FY 1999-2000

Average, Median, and Beginning Professional Salaries in ARL University Libraries; Summary of Rankings, FYs 1996-1997 to 1999-2000

Distribution of Professional Staff in ARL University Libraries by Salary and Position, FY 1999-2000

$14 \quad 16$ Distribution of Professional Staff in ARL University Libraries by Salary, Sex, and Position, FY 1999-2000

$15 \quad 17 \quad$ Number and Average Salaries of ARL University Librarians by Position and Sex, FY 1999-2000

$17 \quad 18 \quad$ Number and Average Years of Experience of ARL University Librarians by Position and Sex, FY 1999-2000 


\begin{tabular}{|c|c|c|}
\hline Old & New & \\
\hline 19 & 19 & $\begin{array}{l}\text { Number and Average Salaries of ARL University Librarians } \\
\text { by Years of Experience and Sex, FY 1999-2000 }\end{array}$ \\
\hline 21 & 20 & Average Salaries of ARL University Librarians by Years of Experience, FY 1999-2000 \\
\hline 22 & 21 & $\begin{array}{l}\text { Number and Average Salaries of ARL University Librarians } \\
\text { by Position and Type of Institution, FY 1999-2000 }\end{array}$ \\
\hline $22 b$ & 22 & $\begin{array}{l}\text { Years of Experience of ARL University Librarians } \\
\text { by Position and Type of Institution, FY 1999-2000 }\end{array}$ \\
\hline 23 & 23 & $\begin{array}{l}\text { Number and Average Salaries of ARL University Librarians } \\
\text { by Position and Size of Professional Staff, FY 1999-2000 }\end{array}$ \\
\hline $23 b$ & 24 & $\begin{array}{l}\text { Years of Experience of ARL University Librarians } \\
\text { by Position and Size of Professional Staff, FY 1999-2000 }\end{array}$ \\
\hline 24 & 25 & $\begin{array}{l}\text { Average Salaries of ARL University Librarians } \\
\text { by Position and Geographic Region, FY 1999-2000 }\end{array}$ \\
\hline
\end{tabular}

\section{US ARL UNIVERSITY LIBRARIES}

N/A $26 \quad$ Average Salaries of US ARL University Librarians

by Position and Years of Experience, FY 1999-2000

\section{US ARL UNIVERSITY LIBRARIES}

Number and Average Salaries of Minority US ARL University Librarians by Position and Sex, FY 1999-2000

Number and Average Years of Experience of Minority US ARL University Librarians by Position and Sex, FY 1999-2000

N/A $29 \quad$ Number and Average Salaries of US ARL University Librarians by Years of Experience and Sex, FY 1999-2000

Number and Average Salaries of Minority US ARL University Librarians by Years of Experience and Sex, FY 1999-2000

\section{Canadian ARL University Libraries}

Filled Positions; Average, Median, and Beginning Professional Salaries; and Average Years of Professional Experience in Canadian ARL University Libraries, FY 1999-2000

Number and Average Salaries of Canadian ARL University Librarians by Position and Sex, FY 1999-2000

Number and Average Years of Experience of Canadian ARL University Librarians by Position and Sex, FY 1999-2000

Number and Average Salaries of Canadian ARL University Librarians by Years of Experience and Sex, FY 1999-2000

\section{ARL University Medical Libraries}

Filled Positions; Average, Median, Beginning Professional Salaries; and Average Years of Professional Experience in ARL University Medical Libraries, FY 1999-2000 


\begin{tabular}{|c|c|c|}
\hline Old & New & \\
\hline 31 & 37 & $\begin{array}{l}\text { Median Professional Salaries in ARL University Medical Libraries; } \\
\text { Rank Order Table, FY 1999-2000 }\end{array}$ \\
\hline 32 & 38 & $\begin{array}{l}\text { Average Professional Salaries in ARL University Medical Libraries; } \\
\text { Rank Order Table, FY 1999-2000 }\end{array}$ \\
\hline 33 & 39 & $\begin{array}{l}\text { Number and Average Salaries of ARL University Medical Librarians } \\
\text { by Position and Sex, FY 1999-2000 }\end{array}$ \\
\hline 34 & 40 & $\begin{array}{l}\text { Number and Average Years of Experience of ARL University Medical Librarians } \\
\text { by Position and Sex, FY 1999-2000 }\end{array}$ \\
\hline 35 & 41 & $\begin{array}{l}\text { Number and Average Salaries of ARL University Medical Librarians } \\
\text { by Years of Experience and Sex, FY 1999-2000 }\end{array}$ \\
\hline & & ARL UNIVERSITY LAW LIBRARIES \\
\hline 36 & 42 & $\begin{array}{l}\text { Filled Positions; Average, Median, Beginning Professional Salaries; and Average } \\
\text { Years of Experience in ARL University Law Libraries, FY 1999-2000 }\end{array}$ \\
\hline 37 & 43 & $\begin{array}{l}\text { Beginning Professional Salaries in ARL University Law Libraries; } \\
\text { Rank Order Table, FY 1999-2000 }\end{array}$ \\
\hline 38 & 44 & $\begin{array}{l}\text { Median Professional Salaries in ARL University Law Libraries; } \\
\text { Rank Order Table, FY 1999-2000 }\end{array}$ \\
\hline 39 & 45 & $\begin{array}{l}\text { Average Professional Salaries in ARL University Law Libraries; } \\
\text { Rank Order Table, FY 1999-2000 }\end{array}$ \\
\hline 40 & 46 & $\begin{array}{l}\text { Number and Average Salaries of ARL University Law Librarians } \\
\text { by Position and Sex, FY 1999-2000 }\end{array}$ \\
\hline 41 & 47 & $\begin{array}{l}\text { Number and Average Years of Experience of ARL University Law Librarians } \\
\text { by Position and Sex, FY 1999-2000 }\end{array}$ \\
\hline 42 & 48 & $\begin{array}{l}\text { Number and Average Salaries of ARL University Law Librarians } \\
\text { by Years of Experience and Sex, FY 1999-2000 }\end{array}$ \\
\hline
\end{tabular}

